\title{
Gelatinase-mediated Migration and Invasion of Cancer Cells
}

\author{
Mikael Björklund
}

Department of Biological and Environmental Sciences, Faculty of Biosciences, and Viikki Graduate School in Biosciences, University of Helsinki

\section{Academic Dissertation}

To be presented for public criticism, with the permission of the Faculty of Biosciences, University of Helsinki, in the Lecture Hall 2402 at Viikki Biocenter Bldg. 3, Helsinki, on October $22^{\text {nd }}, 2004$, at 12 p.m.

Helsinki 2004 
Supervised by: $\quad$ Docent Erkki Koivunen

Department of Biological and Environmental Sciences

University of Helsinki

Helsinki, Finland

Reviewed by: $\quad$ Professor Jorma Keski-Oja.

Departments of Pathology and Virology

Biomedicum and Haartman Institute

University of Helsinki

Helsinki, Finland

and

Professor Jyrki Heino

Department of Biochemistry and Food Chemistry

University of Turku

Turku, Finland

Opponent: $\quad$ Professor Antti Vaheri

Department of Virology

Haartman Institute

University of Helsinki

Helsinki, Finland

ISSN: $1239-9469$

ISBN: 952-10-2024-5 (printed)

952-10-2025-3 (PDF)

Yliopistopaino

Helsinki 2004 


\section{CONTENTS}

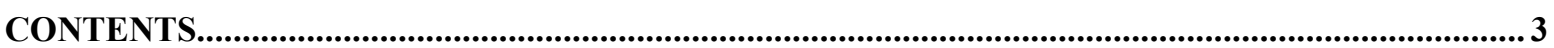

LIST OF ORIGINAL PUBLICATIONS ....................................................................................................5

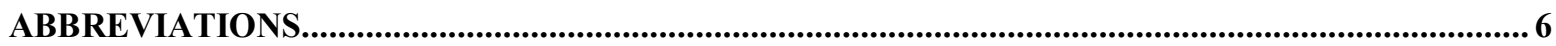

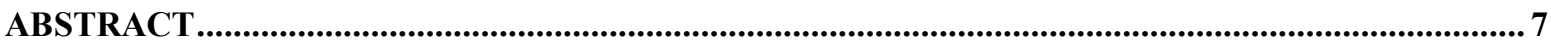

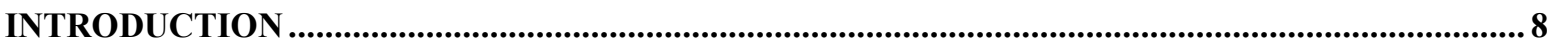

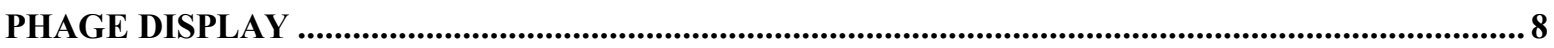

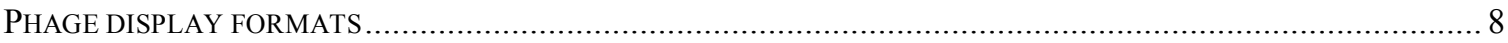

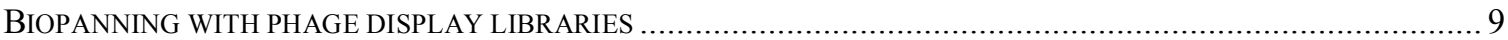

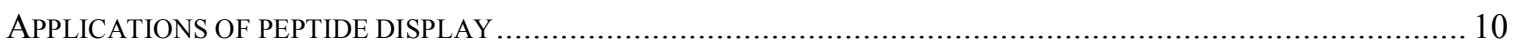

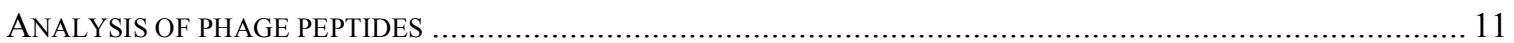

Recombinant protein expression using self-splicing inteins .............................................. 12

BIOSYNTHETIC METHODS FOR INCORPORATION OF NOVEL AMINO ACIDS .......................................... 12

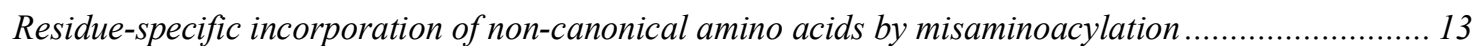

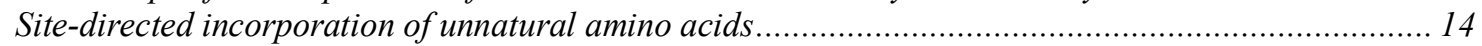

CURRENT APPROACHES TO INCREASE THE CHEMICAL DIVERSITY OF BIOLOGICAL DISPLAY LIBRARIES.......... 15

GELATINASES AND OTHER MATRIX METALLOPROTEINASES ................................................. 17

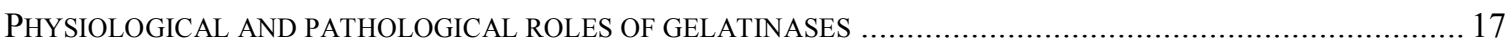

STRUCTURAL FEATURES OF MATRIX METALLOPROTEINASES ..................................................... 18

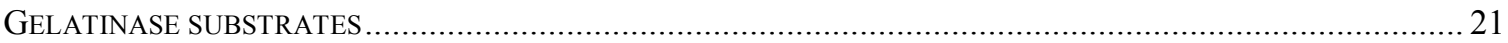

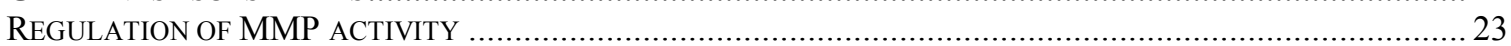

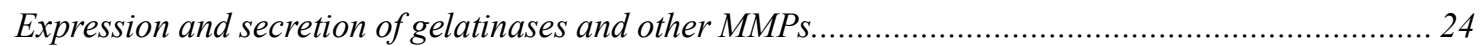

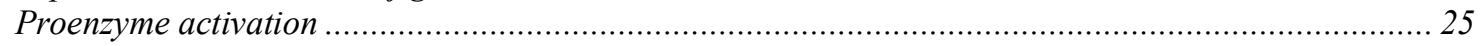

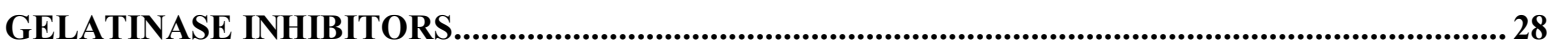

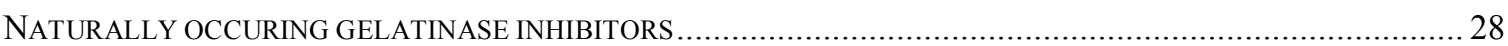

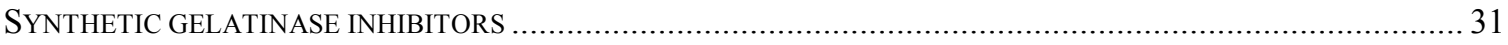

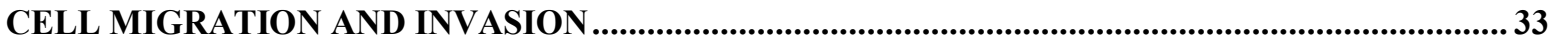

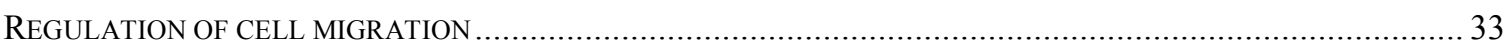

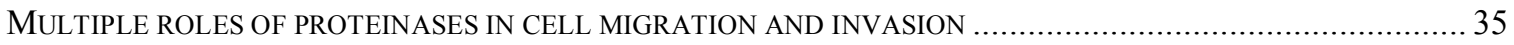

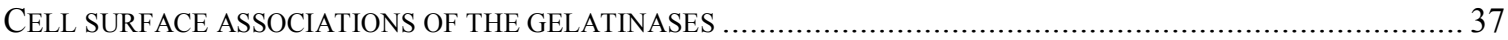

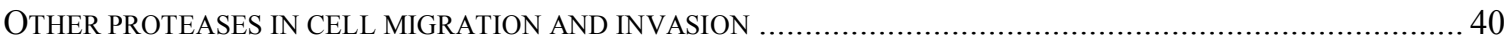

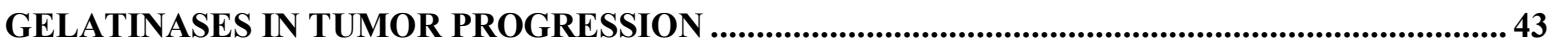

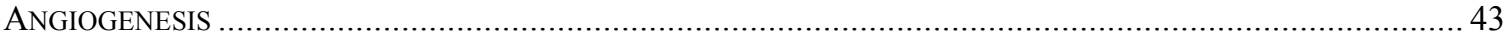

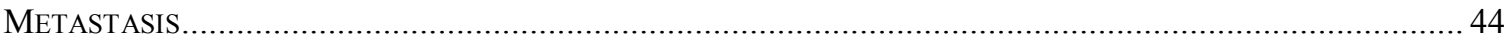

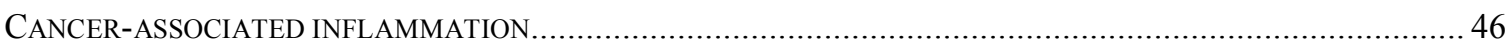

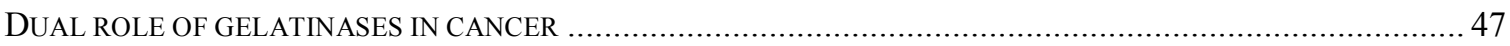

THERAPEUTIC POSSIBILITIES WITH THE MMP INHIBITORS ....................................................... 48

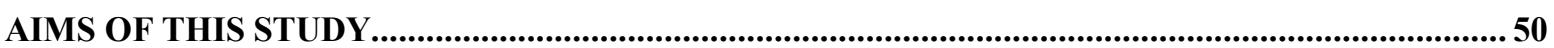

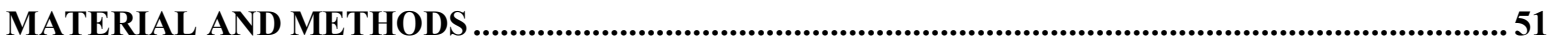

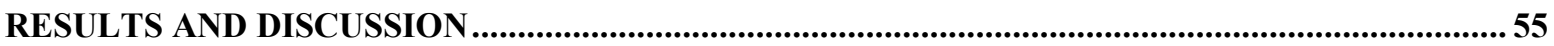

BIOSYNTHESIS OF PHAGE DISPLAY PEPTIDES (I, III-IV) ................................................................. 55

INCREASE OF SERUM STABILITY OF GELATINASE INHIBITOR PEPTIDE CTT BY INCORPORATION OF A 5-

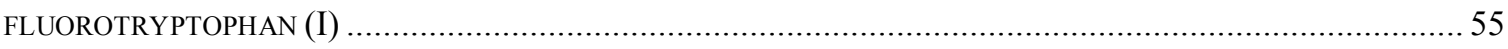

INCORPORATION OF AMINO ACID ANALOGUES INTO PHAGE DISPLAY LIBRARIES (II) .................................56 
INHIBITION OF MMP-9 INTERACTION WITH $\alpha_{M}$ INTEGRIN I DOMAIN BY PHAGE DISPLAY PEPTIDES CONTAING

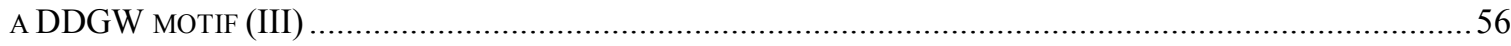
IDENTIFICATION OF DOMAIN-SPECIFIC LIGANDS OF MMP-9 THAT INHIBIT TUMOR CELL MIGRATION AND

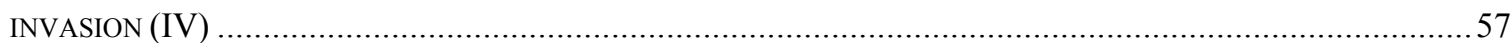

MMP-9 INTERACTS WITH THE UROKINASE-PLASMINOGEN ACTIVATOR RECEPTOR AND THE INTEGRIN $\beta$ CHAIN (IV). ...58

IDENTIFICATION OF A SMALL MOLECULE INHIBITOR OF $\alpha_{\mathrm{M}} \beta_{2}$ INTEGRIN-DEPENDENT LEUKEMIA CELL 59

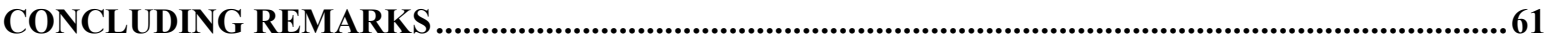

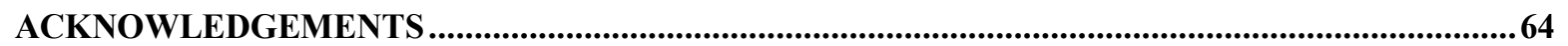

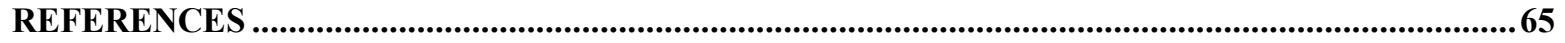




\section{LIST OF ORIGINAL PUBLICATIONS}

This thesis is based on the following original articles, which are referred to by their Roman numerals in the text.

Björklund, M., Valtanen, H., Savilahti, H. and Koivunen, E: Use of intein-directed peptide biosynthesis to improve serum stability and bioactivity of a gelatinase inhibitory peptide. Combinatorial Chemistry and High Throughput Screening 6, 29-35, 2003.

II

Björklund, M. and Koivunen, E: Steps towards phage display libraries with an extended amino acid repertoire. Letters in Drug Design \& Discovery 1, 163-167, 2004.

III

Stefanidakis, M., Björklund, M., Ihanus, E., Gahmberg, C. G. and Koivunen, E: Identification of a negatively charged peptide motif within the catalytic domain of progelatinases that mediates binding to leukocyte $\beta 2$ integrins. Journal of Biological Chemistry, 278, 34674-84, 2003.

IV

Björklund, M., Heikkilä, P. and Koivunen, E: Peptide inhibition of catalytic and noncatalytic activities of matrix metalloproteinase-9 blocks tumor cell migration and invasion. Journal of Biological Chemistry, 279, 29589-97, 2004.

V

Björklund, M. and Koivunen, E: A small-molecule stabilizing the active conformation of the $\alpha_{M}$ integrin I domain inhibits leukemia cell migration. Submitted, 2004.

The original articles have been reprinted with the permission of the publishers. 


\section{ABBREVIATIONS}

ADAM

ADAMTS

APMA

bFGF

CAM

CBD

CTT

CRV

DDGW

ECM

EGF

EMMPRIN

ENA-78

FAK

GCP-2

GPI

GRO- $\alpha$

GST

$\mathrm{HGF} / \mathrm{SF}$

ICAM

I domain

IFN

IGF

IL

IP-10

LRP

MAPK

MIG

MMP

NMR

$\mathrm{PDBu}$

PDGF

PF-4

PPC

RECK

SDF-1

SIBLINGs

SPARC

TIMP

TSP

UPA

UPAR

VEGF a disintegrin and metalloproteinase

a disintegrin and metalloproteinase with a thrombospondin motif aminophenyl mercuric acetate, an activator of MMPs

basic fibroblast growth factor

chicken chorionallantoic membrane

collagen-binding domain

gelatinase inhibitor peptide CTTHWGFTLC

MMP-9 C-terminal domain binding peptide CRVYGPYLLC

$\alpha_{\mathrm{M} / \mathrm{L}}$ I domain ligand peptide ADGACILWMDDGWCGAAG

extracellular matrix

epidermal growth factor

extracellular matrix metalloproteinase inducer

epithelial-cell derived neutrophil activating peptide-78

focal adhesion kinase

granulocyte chemotactic protein-2

glycophosphatidyl inositol

growth-regulated oncogene- $\alpha$

glutathione-S-transferase

hepatocyte growth fator/scatter factor

intercellular adhesion molecule

integrin ligand-binding inserted domain

interferon

insulin-like growth factor

interleukin

interferon-inducible protein-10

low-density lipoprotein receptor-related protein

mitogen-activated protein kinase

monokine induced by interferon- $\gamma$

matrix metalloproteinase

nuclear magnetic resonance

$4 \beta$-phorbol-12, 13-dibutyrate, a phorbol ester

platelet-derived growth factor

platelet factor-4

gelatinase CBD-ligand peptide ADGACGYGRFSPPCGAAG

reversion-inducing cysteine-rich protein with Kazal motifs

stromal-cell derived factor-1

small integrin-binding ligand $\mathrm{N}$-linked glycoproteins

secreted protein, acidic and rich in cysteines

tissue inhibitor of matrix metalloproteinases

thrombospondin

urokinase type plasminogen activator

urokinase type plasminogen activator receptor

vascular endothelial growth factor 


\section{ABSTRACT}

Display of random peptides on the surface of filamentous bacteriophage allows identification of ligands to virtually any target. We have examined here new strategies to improve the phage display-derived peptides and to expedite their development into drugleads. This has been achieved by integrating phage display with a recombinant peptide expression and by incorporating amino acid analogues into soluble peptides and peptides displayed on phage. We have also utilized phage for screening a small-molecule compound library.

We have studied the migration and invasion mechanisms of neoplastic cells by inhibition of matrix metalloproteinase (MMP)-2 and -9 functions with the phage display derived peptides. In addition to improving the stability of a gelatinase inhibitor peptide, we have found several new strategies to interfere with gelatinase functions. A peptide mimicking a sequence in the catalytic domain of the gelatinases bound to the $\alpha_{M}$ and $\alpha_{L}$ I domains of the leukocyte integrins and inhibited leukemia cell migration. Biopanning with proMMP-9 identified a novel and selective inhibitor of gelatinases. By similarity to this peptide we identified a gelatinase-recognition motif in the extracellular matrix proteins fibronectin and vitronectin. A peptide blocking the interaction of the C-terminal domain of MMP-9 with cell surface $\beta_{5}$ integrins was found to be an efficient inhibitor of HT1080 fibrosarcoma cell migration and invasion although it did not affect MMP-9 activity in vitro. The binding site of the C-terminal domain of MMP-9 in the integrin could be identified by sequence similarity, and it was located to an activation epitope in the stalk of the integrin $\beta$ chain, and not the typical integrin ligand-binding region. The $\mathrm{C}$-terminal domain-binding peptide also blocked the migration of leukemia cells by binding to the $\beta_{2}$ integrin subunit and inhibited the plasminogen/MMP-3 dependent activation of MMP-9. Urokinase plasminogen activator (uPA) and the urokinase receptor ( $\mathrm{UPAR}$ ) are responsible for the cell surface activation of plasminogen and critically involved in cell migration and tumor metastasis. We observed an interaction of UPAR with MMP-9 and found that UPAR was cleaved by MMP-9. In addition, the C-terminal domain-binding peptide inhibited human tumor xenograft growth in a mouse model indicating a novel utility for MMP-9 inhibitors, which do not inhibit enzymatic activity.

As an alternative to the modification of phage display peptides, a small-molecule compound library was screened to identify chemicals that would compete with the $\alpha_{M}$ I domain-binding peptide. This screen identified a novel compound that potently inhibited phage binding to the $\alpha_{M}$ integrin I domain, but did not inhibit but rather increased proMMP-9 binding to this domain. In addition, we found that this compound increased the resistance of leukemia cells to detachment from $\alpha_{M} \beta_{2}$ integrin ligands and that this compound potently inhibited $\alpha_{M} \beta_{2}$ integrin-dependent leukemia cell migration without affecting gelatinase activity.

In summary, we have identified molecular details for several interactions between MMP-9 and various cell surface and extracellular matrix molecules. Our results show that these interactions plays an important role in the motility of neoplastic cells and that prevention of these interactions inhibits cancer cell migration and invasion. However, our research with the chemical inhibitor of leukemia cell migration indicates that gelatinase-independent mechanisms for cell migration exist implicating a possible need for additional treatment strategies to completely inhibit cancer cell motility. 


\section{INTRODUCTION}

\section{PHAGE DISPLAY}

\section{Phage display formats}

The bacteriophage M13, Fd and other related filamentous bacteriophage are the most commonly used vectors for phage display, although other phage such as T7 phage and $\lambda$ phage have also been successfully used (Kuwabara et al., 1997; Laakkonen et al., 2002). The filamentous bacteriophage are single-stranded DNA containing viruses infecting many gram-negative bacteria, which harbor a F pilus. The virions are rod-like, the width being approximately $6.5 \mathrm{~nm}$ and the length $900-2000 \mathrm{~nm}$, depending on the genome size (Glucksman et al., 1992; Model and Russel, 1988). The filamentous phage genome is relatively compact containing eleven genes, which encode for the capsid proteins and proteins required for DNA replication and virion assembly (Model and Russel, 1988). The single stranded DNA is packed into virions on the membrane of the bacterial host with the aid of assembly proteins of the phage and the host proteins such as thioredoxin (Marciano et al., 1999; Russel and Model, 1985). The completed phage particle contains a single major coat protein, pVIII present in about 2900 copies. Additionally, one end of the phage particle contains two minor coat proteins pIII and pVI and the other end pVII and pIX proteins in about 2-5 copies each (Makowski, 1992, Uppala and Koivunen, 2000).

In the mid-1980's George Smith demonstrated that foreign polypeptides could be expressed as a fusion with the minor coat protein pIII and used to find information about antibody epitopes by affinity selection of the phage that bound to an antibody (Parmley and Smith, 1988; Smith, 1985). The novelty of this method was that each particle carried the coding information of the displayed polypeptide in their genome thus facilitating the identification of the binding epitope. Individual antibody-binding phage could be easily identified using standard microbiological techniques and amplified in E. coli to isolate sufficient quantities of DNA for sequencing. By cloning oligonucleotides containing degenerate codons, a random library of peptides was constructed, each phage carrying a single peptide on the coat protein (Scott and Smith, 1990). These unconstrained, completely random peptide libraries are typically referred as linear peptide libraries. It is also possible to constrain the peptide libraries by fixing certain amino acids in predetermined positions. For example, cyclic disulfide-constrained libraries are obtained when two cysteines flank the otherwise random sequence. Due to the structural constrain, the cyclic peptides are typically of higher affinity than the more flexible linear peptides (Koivunen et al., 1993; McLafferty et al., 1993).

Antibodies, enzymes, growth factors and many other large proteins have also been expressed as a fusion on the minor coat protein pIII (Lowman et al., 1991; McCafferty et al., 1990; McCafferty et al., 1991). The display of antibodies and other larger proteins can be used to isolate high-affinity binders or to isolate variants with specific properties. Although antibodies can be efficiently displayed in a single chain form and as Fab fragments, the display efficiency of other proteins varies considerably limiting the use of filamentous phage in cDNA library screenings (Hufton et al., 1999). Other coat proteins of the phage have also been used for display of peptides and proteins. The most commonly used alternative is the pVIII major coat protein (Felici et al., 1991). The advantage of the pVIII display is that the peptides are displayed in much higher number leading to avidity effects. The disadvantage is that the packaging of the phage is often disturbed especially by 
large insertions. Thus, hybrid phage with both wild type and recombinant $p$ VIII proteins are commonly used (Greenwood et al., 1991). All the other coat proteins of the filamentous bacteriophage can and have been utilized for phage display. The pVII and pIX minor coat proteins have been used together for two-chain antibody display (Gao et al., 1999) and pVI display for peptide and cDNA display. In contrast to the pIII and pVIII display systems, the $\mathrm{C}$-terminus of the $\mathrm{pVI}$ protein projects outwards and allows display of peptides and proteins with a free C-terminus (Hufton et al., 1999). Unexpectedly, the C-terminus of the pIII and pVIII proteins have also been successfully used for peptide display in a phagemid format (Fuh et al., 2000; Fuh and Sidhu, 2000).

\section{Biopanning with phage display libraries}

Basically, phage display is based on affinity selection to enrich specifically binding phage over a huge excess of irrelevant phage (Figure 1). In the simplest form phage are allowed to bind to an immobilized target, unbound phage are washed away and the bound phage are eluted using a low $\mathrm{pH}$ buffer or a known competitive ligand. It is also possible to use biotinylated or otherwise tagged target proteins and allow them to react with the phage in solution followed by affinity capture of the target and the bound phage. The eluted phage are then allowed to infect bacteria and are then amplified and recovered using a polyethylene glycol-sodium chloride precipitation. Successive rounds with the enriched phage preparations are done until a sufficient enrichment of specifically bound phage is achieved (Koivunen et al., 1999). The most critical issue in biopanning is a careful design and control over the selection conditions, because the method selects for the best binders in the used condition, which is not necessarily a physiologically relevant one.

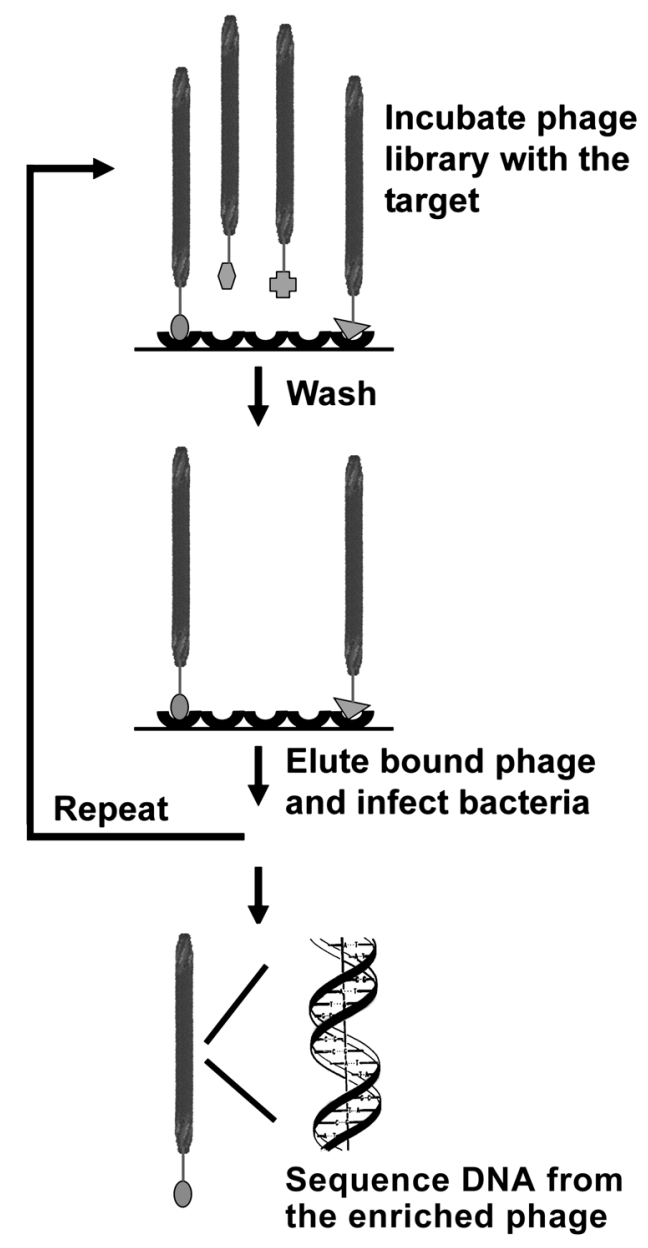

Figure 1. The biopanning procedure using an immobilized target molecule. The biopanning cycle consisting of binding, washing and elution steps is repeated until a sufficient enrichment of specifically bound phage is achieved, usually after $2-5$ rounds. 
It is also essential that the target is in an active form. In some cases the target protein may not be purified in a native form necessitating the use of specific selection schemes. Indeed, the target molecule does not need to be pure. With an appropriate selection design, it is possible to enrich peptides to a target present in a complex environment such as a cell surface or in a tissue biopsy (Goodson et al., 1994). A subtractive step with a similar prepararation, but lacking the target molecule, for example, cells not expressing the specific receptor or an immunodepleted protein mixture, is used to eliminate non-target binding sequences from the initial library. Thereafter the unbound phage are transferred to the mixture containing the target to enrich the specifically binding clones.

\section{Applications of peptide display}

Although phage display was originally developed to identify antibody epitopes, phage display libraries are now widely used to identify peptide ligands to various proteins and other biomolecules (Goodson et al., 1994; Healy et al., 1995; Koivunen et al., 1999; Koivunen et al., 1993; Koivunen et al., 2001). Phage display is by no means limited to biological targets as peptides binding to semiconducting and magnetic materials can be isolated as well (Mao et al., 2004; Whaley et al., 2000). In an optimal case, isolation of the peptide ligands allows the identification of a binding partner for the target protein together with the interacting sequence. However, when synthetic degenerate peptide libraries are used, the peptide ligands rarely exactly match with a natural protein sequence. In addition, the short peptides commonly show similarities to a wide variety of proteins, and often only three or four amino acid residues in the peptide are directly involved in target binding, whereas the rest may be structurally required or have no specific function. Hence, in the absence of any information about possible binding partners a high rate of false positive interactions are typically obtained from database searches possibly masking the true interactions (Smothers and Henikoff, 2001). Such problems can be avoided by displaying randomly fragmented cDNA (Matthews et al., 2002).

One application of the phage peptide libraries is the mapping of substrate recognition sites for proteases. Protease cleavage sites can be found using substrate phage immobilized on a solid support. Addition of an active protease releases only the phage containing a substrate recognition sequence. These phage can then be amplified and further enriched to determine the optimal substrates for the protease of interest. This technique has been used to map the cleavage sites of subtilisin (Matthews and Wells, 1993), furin (Matthews et al., 1994) and several matrix metalloproteinases (MMPs) (Chen et al., 2002; Deng et al., 2000; Kridel et al., 2001; Kridel et al., 2002; Smith et al., 1995). Not only protease substrates can be found using phage display. It is well established that the phage-display selected peptides commonly bind to biologically relevant sites in the targets such as the catalytic site of enzymes or ligand-binding site of receptors. This is because a small peptide has to form contacts with relatively large areas in the target in order to have sufficient binding affinity. The active sites of the enzymes and the ligand-binding sites of the receptors typically form suitable large clefts, crevices or holes with some flexibility to accommodate the substrates/ligands (Kay and Hamilton, 2001). The peptide CTTHWGFTLC (CTT), which binds to the gelatinases MMP-2 and MMP-9 is a model example of a phage display peptide that also acts as a potent inhibitor (Koivunen et al., 1999). A large number of other enzyme inhibitors have been discovered including enzymes, which normally have non-protein substrates (Kay and Hamilton, 2001). 
Biopanning is by no means limited to in vitro and ex vivo applications. Phage display can be equally well performed in vivo. Here, the phage library is injected into the blood stream of an organism, and the phage are subsequently recovered from the individual organs. Remarkably, this method has permitted the isolation of peptides that home to specific organs in mice without a prior knowledge about the target molecules present in the organ vasculature (Pasqualini and Ruoslahti, 1996). The in vivo display has also been performed in human subjects (Arap et al., 2002) and other organisms such a mosquitos (Ghosh et al., 2001). The in vivo display offers an exciting route to develop peptides homing to specific locations, such as the tumor vasculature (Ruoslahti, 2000). The homing peptides obtained by in vivo display can be used to deliver various cargo such as cytotoxic drugs, proteins, liposomes, imaging agents and viruses into specific sites in an organism (Akerman et al., 2002; Arap et al., 1998; Curnis et al., 2000; Medina et al., 2001; Turunen et al., 2002). The advantage of the targeting approach is that it minimizes the adverse effects of the drugs or other cargo in non-target organs.

\section{Analysis of phage peptides}

One of the current bottlenecks in the phage display selections is the functional analysis of the target-binding sequences. A single phage selection typically yields multiple potential peptide ligands from which the most suitable candidates should be selected. Although the selections could in principle be continued until a single sequence remains, this is rarely a favorable practice as the affinity of the binding sequence is not the only selective force in the biopannings. For example, the effect of the displayed peptides on phage particle production may significantly favor the selection of some sequences. Optimally, one would like to determine all different peptide motifs binding to a target and not to introduce any bias in the selections. Another issue is that the selected peptides may not be sufficiently soluble for the intended purposes. It is thus advantageous to analyze the sequences from the early rounds of selections to get maximal information on the binding sequence motifs and to obtain sufficiently diverse selection of peptides for activity analysis. Furthermore, by alignment of the phage peptides, consensus sequence motifs are obtained and these rather than single sequences can be used to search for putative binding partners allowing filtering of the false positive interactions (Deshayes et al., 2002; Smothers and Henikoff, 2001). However, having a multitude of target binding sequences possesses a practical problem, because one should be able to analyze the peptides for biological activity. Traditionally, phage peptides have been prepared as fusions with larger proteins such as glutathione-Stransferase (GST) (Rajotte et al., 1998) or alkaline phosphatase (Wright et al., 2001). However, with this approach it is often difficult or impossible to obtain peptide concentrations sufficient to demonstrate the biological activity of a peptide such as enzyme inhibition. Furthermore, it is also impossible to analyze the solubility properties of the peptides. Hence, one would preferably analyze a reasonable number of different peptides as such without any fusion tags. Chemical peptide synthesis is rather expensive and often only small quantities of peptides would be sufficient for the initial analysis. Although highthroughput peptide synthesis methods have been developed (Pipkorn et al., 2002), they may not be easily available. In these cases recombinant methods to produce the peptides are a competitive alternative. 


\section{Recombinant protein expression using self-splicing inteins}

Inteins are a group of proteins with intrinsic self-cleavage ability originally found in yeast (Kane et al., 1990), and are the proteinaceous equivalents for introns in RNA. Inteins are cleaved from the precursor proteins with the concomitant joining of the intein-flanking polypeptides (the exteins) to form a functional protein (Paulus, 2000). Due to the controllable autocatalytic trait of the inteins, they are now used as fusion partners for recombinant protein expression (Chong et al., 1997; Chong et al., 1998). The advantage is that it is possible to use the intein in affinity purification and then through a controlled protein cleavage obtain the protein of interest in a native form without any extraneous amino acid sequences. The intein cleavage occurs under mild conditions and avoids the use of proteinases or peptide bond-cleaving chemicals, which often have adverse effects on recombinant proteins (Chong et al., 1997).

The intein cleavage occurs through a series of intramolecular reactions at the intein-extein junction. The rearrangement of the peptide bonds results in the formation of a reactive thioester, which can be cleaved with thiol compounds, such as cysteine or dithiotreitol (Paulus, 2000). Mutant inteins displaying cleavage activity in the absence of thiols have also been isolated (Mathys et al., 1999). The cleavage activity of these proteins can be modulated by $\mathrm{pH}$ and temperature control. An additional benefit of the inteins as a fusion partner is that the unique reactivity of the thioester bond can be exploited for a selective modification of the expressed protein. By utilizing this trait, site-selective biotinylation (Lesaicherre et al., 2002), C-terminal amidation (Cottingham et al., 2001), backbone cyclization via a native peptide bond between the $\mathrm{N}$ - and the C-terminus of the protein (Evans et al., 1999; Scott et al., 1999) and addition of non-natural amino acids (Severinov and Muir, 1998) into recombinant proteins has been accomplished.

The self-cleavage of the inteins makes them attractive fusion partners for peptide expression. Furthermore, minimal inteins with full cleavage activity have only 130-160 amino acids (Derbyshire et al., 1997; Mathys et al., 1999; Wood et al., 1999). With a minimal sized fusion partner, the protein expressing cells do not have to consume their metabolic reserves for expressing large unwanted proteins thus maximising the peptide yield.

\section{Biosynthetic methods for incorporation of novel amino acids}

Phage display has been highly successful in the identification of surrogate ligands for various proteins, because it does not require any structural knowledge about the target. Nor are extensive screenings required as with the chemical compound libraries. Typically a few microtiter wells coated with submicrogram quantities of the target protein are sufficient to yield the desired peptide ligands. However, more and more structural information of the proteins is becoming available and the availability of compound libraries increases together with the development of improved screening methodologies, including the in silico screenings (Bajorath, 2002; Engels and Venkatarangan, 2001). These advancements are significantly simplifying and accelerating the discovery of small-molecule ligands. Furthermore, the inherent proteinaceous nature of the biological display library-derived ligands poses another problem as peptides are often rapidly degraded and/or cleared from the circulation and may require extensive modifications to extend their utility in vivo (Adessi and Soto, 2002; Lien and Lowman, 2003). 
The ability of $E$. coli to incorporate nonproteinogenic amino acids into polypeptides has been known for decades (Cowie and Cohen, 1957; Fenster and Anker, 1969; Hagen et al., 1978; Hagen et al., 1979; Rennert and Anker, 1963), but this property has only recently been exploited for the modification of recombinant proteins. The two main strategies are the residue-specific incorporation of nonnatural amino acids by misaminoacylation of transferRNAs (tRNAs) (Kiick et al., 2001; Kirshenbaum et al., 2002; Niemz and Tirrell, 2001) and the site-directed incorporation, which utilizes non-cognate amber suppressor tRNA/aminoacyl-tRNA synthetase pairs (Noren et al., 1989; Wang et al., 2001). The significance of these methodologies is that by incorporation of nonnatural amino acid residues proteins or peptides can be modulated to have enhanced metabolic and/or thermal stability and/or increased activity (Tang et al., 2001; Tang and Tirrell, 2001). Alternatively, novel functional groups such as fluorescent probes (Cornish et al., 1994), amino acids with side chains containing chemically modifiable groups (Kiick et al., 2002) or photoaffinity labels for cross-linking (Chin et al., 2002) can be added. In the context of phage display the addition of novel amino acids could increase the peptide diversity leading to the discovery of peptides with higher activity and other desired properties, such as increased stability.

In addition to aforementioned two methodologies, it is possibly to modify ribosomes to accept D-amino acid isomers instead of the normal L-amino acids (Dedkova et al., 2003). This approach utilizes ribosomes that have been formed when a mutated $23 \mathrm{~S}$ ribosomal RNA is expressed in high levels in E. coli. These mutant ribosomes, which tolerate D-amino acids can be isolated and used in protein synthesis in vitro. The ribosomes are, to some extent, able to accept amino acids with peptide-backbone modifications. These approaches concentrating on the modification of ribosomes may significantly contribute to the chemical diversity of the in vitro display systems (Frankel et al., 2003). In principle, non-ribosomal peptide synthesis is another possible route for the biosynthesis of highly modified peptides (Cane et al., 1998; Velkov and Lawen, 2003). However, the existing applications have been focused on the modification of naturally-occurring non-ribosomally synthesized peptides and it may be difficult to adopt this methodology for the synthesis of custom peptides.

\section{Residue-specific incorporation of non-canonical amino acids by misaminoacylation}

The misaminoacylation of tRNAs with amino acid analogues requires that the protein expression host, typically E. coli, is auxotrophic for the amino acid to be replaced. This means that the host must be deficient of synthesizing a particular amino acid. Many amino acid auxotrophic bacterial hosts are available or they can be readily prepared by mutagenesis. The misaminoacylation systems rely on the culture of these auxotrophic bacteria in a defined culture medium. Before the induction of protein expression, the bacteria are changed to a culture medium lacking the amino acid to be replaced, and a suitable amino acid analogue is added (Ibba and Hennecke, 1995; Kiick et al., 2001; Tang et al., 2001). The tRNAs to the particular amino acid are misaminoacylated with the analogues and subsequently incorporated into the expressed proteins. One noteworthy application of this method is the ability to incorporate selenomethionine into proteins. This has significantly helped in solving X-ray structures by the multiwavelenght anomalous diffraction method (Budisa et al., 1995; Hendrickson et al., 1990).

Although the residue-specific method is simple and allows the incorporation of a wide variety of amino acid analogues, the disadvantages of this methodology are obvious. First, a 
defined culture medium is needed, which typically results in significantly decreased protein yields. Second, the amino acid analogues must be structurally similar to the parental amino acid so that the aminoacyl-tRNA synthetase will accept them and attach them to the tRNAs. Third, this method replaces a single amino acid rather than expands the amino acid repertoire. One additional issue of concern is that some amino acid analogues are toxic to the cells thus reducing their utility in protein expression, although tolerant mutant bacteria can be isolated (Bacher and Ellington, 2001). Of the many amino acid analogues that can be incorporated by misaminoacylation, fluorinated analogues of tyrosine, tryptophan, phenylanine, and leucine have been the most widely used (Hagen et al., 1978; Minks et al., 1999; Minks et al., 2000; Rennert and Anker, 1963). Indeed, fluorine substitution offers many advantages. Due to the small size of the fluorine atom, the fluorinated analogues fit well to the active site of the aminoacyl-tRNA synthetases resulting in a high misaminoacylation rate. The fluorine atom also changes fluorescence properties of the aromatic amino acids allowing monitoring of the chemical environment of these residues (Minks et al., 2000). Additionally, the fluorine-substituted amino acids are more hydrophobic than the normal amino acids (Yoder and Kumar, 2002). As a result, proteins with fluoroamino acids often show an increase in thermal stability (Tang et al., 2001; Tang and Tirrell, 2001) and/or increased resistance to proteases and improved bioavailability (Hsieh et al., 1987). For these reasons, fluorine substitutions are also used in chemical compounds as a final push to increase their activity as exemplified by the MMP- $2 / \alpha_{\mathrm{V}} \beta_{3}$ integrin interaction-inhibiting molecule (Boger et al., 2001).

The constrains of the misaminoacylation system can be relaxed in several ways. Significant incorporation of structurally diverse phenylalanine analogues was achieved by overexpression of the wild type aminoacyl-tRNA synthetase (Kiick et al., 2000). Another strategy involves mutant synthetases with an enlarged substrate-binding site to accommodate those analogues that would not otherwise fit to the active site (Ibba and Hennecke, 1995). A third strategy is to modify the hydrolytic editing activity of the aminoacyl-tRNA synthetases so that the misaminocylation is not recognized as an error (Doring et al., 2001). An interesting modification of the misaminoacylation system is not to replace a single amino acid with an analogue, but to re-assign only a single codon to code for an analogue and thus break the degeneracy of the genetic code (Kwon et al., 2003). This approach may become highly useful as it does not replace an amino acid completely but expands the amino acid repertoire.

\section{Site-directed incorporation of unnatural amino acids}

In the site-directed approach the aim is to modify a single site in the protein rather than replace all amino acid residues. The key requirements for the site-directed incorporation of amino acid analogues are 1) a codon assigned for the site-selective insertion, 2) a tRNA that does not interact with the endogenous aminoacyl-tRNA synthetases, and 3) a method to acylate the corresponding tRNA with a desired amino acid analogue (reviewed by AnthonyCahill and Magliery, 2002). Typically, the codon that is re-assigned for the insertion is one of the three existing stop codons UAG, amber; UGA, opal; or UAA, ochre, the amber suppression being the most commonly used. It is also possible to assign four base-pair codons as a signal for the analogue incporporation (Magliery et al., 2001). The first sitedirected amino acid incorporation methods relied on chemically acylated tRNAs, which were added to the in vitro translation systems (Noren et al., 1989). Subsequently, through microinjection of aminoacylated tRNAs, membrane proteins could be tagged in vivo with 
unnatural amino acids in Xenopus oocytes (Nowak et al., 1995). The next step was to make this system even more simple and effective by selecting for aminoacyl-tRNA synthetases, which could aminoacylate the tRNAs in vivo. As mentioned above, this required that the new aminoaminoacyl-tRNA synthetase could not use any naturally occurring amino acid as a substrate. These goals were achieved by the preparation of aminoacyl-tRNA synthetase mutant libraries from yeast and archaebacteria, and double-selection schemes with a negative and positive selection step to eliminate those enzymes that were capable of using naturally occurring amino acids and enriching those that were able to utilize the unnatural ones (Liu and Schultz, 1999).

These findings and technological developments have led to the site-directed amino acid incorporation into proteins expressed in bacteria (Furter, 1998; Wang et al., 2001). A further refinement of this system is to evolve the bacteria to autonomouly synthesize the required amino acid analogue. A bacterial strain that contains the aminoacyl-synthetase incorporating $p$-aminophenylalanine and the biosynthetic gene to produce the $p$-aminophenylanine has been developed (Mehl et al., 2003). One of the interesting applications of the site-specific incorporation is the possibility to attach glycosyl groups into E. coli proteins (Liu et al., 2003; Zhang et al., 2004). Such an approach could be very useful for the biotechnology industry due to the simplicity and efficacy of $E$. coli expression. In addition, the sitedirected amino acid analogue incorporation can also be directly applied to eukaryotic expression systems, including mammalian cells (Chin et al., 2003; Sakamoto et al., 2002).

The residue- and site-specific strategies can be viewed as two complementary methods. Whereas the global misaminoacylation strategy aims to change the overall properties of the proteins, the site-directed incorporation strategy allows more subtle and specialized changes in the proteins (Link et al., 2003). It can be speculated that a combination of these strategies could be used to produce extensively modified recombinant proteins for pharmaceutical and other applications.

\section{Current approaches to increase the chemical diversity of biological display libraries}

The application of the amino acid analogue incorporation technologies into biological display libraries is expected to combine the beneficial features of biological display libraries and combinatorial chemistry, namely the powerful biological selections with the large chemical diversity. There has already been significant progress towards increasing the chemical diversity of the biological display libraries beyond the twenty canonical amino acids.

Using in vitro phosphorylation of phage display peptide libraries, kinase substrates have been isolated. In this approach, phage that carry a kinase recognition sequence are phosphorylated and are enriched using phosphospecific antibodies (Gram et al., 1997; Schmitz et al., 1996). A similar approach has been utilized to identify phosphatase substrate sequence specificities (Walchli et al., 2004). Another in vitro amino acid modification approach is the ligation of synthetic unnatural amino acid containing peptides into phage displayed partially randomized proteins (Dwyer et al., 2000). Selenocysteine can be incorporated into phage particles by using a specific, naturally occurring selenocysteine insertion sequence. The selenocysteine residue can be selectively alkylated without affecting other residues including cysteines, thus offering a possibility for a site-specific modification 
of a single amino acid in the displayed polypeptide (Sandman and Noren, 2000). It is also conceivable that the other noncanonical proteinogenic amino acid, pyrrolysine, could be similarly incorporated into phage particles (Namy et al., 2004; Srinivasan et al., 2002). The site-specific amino acid analogue incorporation method has also been applied to phage display. Although the purpose was to use phage as a tool to select for potent amber suppressor tRNA/aminoacyl-tRNA synthetase pairs for efficient incorporation of unnatural amino acids (Pastrnak and Schultz, 2001), this work demonstrates the feasibility of this approach in the context of phage display. Similarly, the misaminoacylation method can be utilized for the incorporation of amino acid analogues into phage proteins. This was already demonstrated in the 1970's, when fluorinated tyrosine and phenylalanine analogues were added to the major coat protein of the M13 phage for NMR analysis (Dettman et al., 1982; Hagen et al., 1978; Hagen et al., 1979). The most advanced approach of increasing the chemical diversity of the phage display libraries involves attachment of a synthetic compound library on phage particles in such a manner that information about the chemical structure is encoded in the phage genome. Using this approach, folate receptor binding compounds were identified (Woiwode et al., 2003).

Ribosome display, tRNA display and mRNA display systems also have the potential to incorporate various amino acid analogues, either by sense or nonsense suppression or chemical derivatization (Frankel et al., 2003). Using the latter method, a peptide-penicillin library was constructed and screened for active penicillin-derivatives (Li and Roberts, 2003). Currently these in vitro display systems have the largest potential to incorporate amino acid analogues, because structurally diverse amino acid analogues can be conveniently linked to the tRNAs by chemical means, and because the in vitro systems do not suffer from toxicity problems caused by the nonnatural amino acids.

A particularly exciting combination of phage display and small-molecule compound libraries is to first select for peptides binding to a target protein and then screen a smallmolecule library for compounds that compete with the phage peptide binding. If reasonably diverse compound libraries are available, this may be the most straightforward path from the phage display peptides to drug candidates as it circumvents the need for the tedious chemical modifications of the peptides. The feasibility of this approach was verified in three different binding assays using phage display-derived peptides and known chemical inhibitors of Haemophilus influenzae tyrosyl-tRNA synthetase (Hyde-DeRuyscher et al., 2000). A library of 250000 compounds has now been screened using this methodology to identify E. coli FtsZ/ZipA protein-protein interaction inhibitors. Among the screened compounds, 29 hits were found (Kenny et al., 2003). This approach offers a direct way to assay for small molecules in the absence of structural, or practically any other information about the target protein. In addition, target validation can be conveniently done with the peptides before conducting large screening programs to search for the small-molecule compounds (Kay et al., 1998). 


\section{GELATINASES AND OTHER MATRIX METALLOPROTEINASES}

The gelatinases A and B, also known as matrix metalloproteinase- 2 and -9 or type IV collagenases are members of the matrix metalloproteinase family. The matrix metalloproteinases are a group of zinc-dependent metalloenzymes containing about 25 members in vertebrates. These enzymes participate in the turnover of extracellular matrix (ECM) and together the MMPs are able to degrade any of the matrix components (Sternlicht and Werb, 2001). The MMPs are not only involved in the mechanical removal of structural proteins in the extracellular matrix. They are also able to regulate multiple cellular functions including cell growth, apoptosis, angiogenesis, invasion, metastasis and immune response by cleaving growth factor-precursors, cell adhesion molecules and other bioactive proteins (Egeblad and Werb, 2002). Of the MMPs, a specific subset, the gelatinases (MMP-2 and MMP-9) have been intensively studied in cancer and other diseases. MMP-2 is abundantly expressed in normal fibroblasts, endothelial and epithelial cells as well as in many transformed cells (Giannelli et al., 1997; Hipps et al., 1991; Partridge et al., 1997; Vartio and Vaheri, 1981). MMP-9 expression is observed in normal leukocytes as well as in transformed cells (Murphy et al., 1980; Sopata and Wize, 1979; Vartio et al., 1982). The genes encoding the gelatinases have been cloned (Huhtala et al., 1990; Huhtala et al., 1991) and these enzymes can be purified with gelatin-affinity chromatography (Hibbs et al., 1985; Johansson and Smedsrod, 1986; Vartio and Vaheri, 1981; Vartio et al., 1982). In addition, gelatin zymography is a simple and highly sensitive technique, which allows relatively specific detection of the gelatinases and their activation status in biological samples (Hibbs et al., 1985). This property has significantly aided in linking the gelatinases into various biological processes. MMP-2 and MMP-9 are highly similar enzymes in many respects, but significant differences exist in the regulation of expression, glycosylation, proenzyme activation and substrate selectivity. For example, MMP-2 is a 72-kDa nonglycosylated protein, whereas the 92-kDa MMP-9 contains two N-glycosylated sites in the prodomain and the catalytic domain (Kotra et al., 2002) and a number of O-linked glycans (Mattu et al., 2000; Rudd et al., 1999). Furthermore, MMP-9 exists in plasma as a monomer, complexed with neutrophil lipocalin and as a dimer, whereas MMP-2 is strictly monomeric. Despite their largely overlapping functions, MMP-2 and MMP-9 may even have opposing biological activity as illustrated by the finding that MMP-2 promotes platelet aggregation, but MMP-9 inhibits the same process (Fernandez-Patron et al., 1999).

\section{Physiological and pathological roles of gelatinases}

Gelatinases play a role in a wide variety of physiological and pathological conditions, among which their role in cancer has been the most extensively studied. The gelatinases are required in invasive prosesses during reproduction, growth and development, leukocyte mobilization and inflammation, and wound healing. Increased gelatinase activity has been observed in a variety of pathological conditions including cancer, inflammation, infective diseases, degenerative diseases of the brain and vascular diseases (Van den Steen et al., 2002).

During reproduction the cells of the implanting embryo secrete gelatinases and other MMPs (Alexander et al., 1996; Behrendtsen et al., 1992). Consequently in some MMP-9 knockout mice strains a reduced breeding efficiency has been observed (Dubois et al., 2000). Although MMPs are widely expressed in the developing embryos, all single MMP knockout animals generated so far are viable, with only minor developmental defects. The MMP-9 
deficient mice show a delayed vascularization and ossification of the hypertrophic zones in cartilage resulting in moderate skeletal abnormalities. This phenotype is similar, athough less severe than the phenotype of the membrane type (MT)1-MMP knockout mice (Holmbeck et al., 1999; Vu et al., 1998). Despite the high level expression of MMP-9 in leukocytes, no major immunodeficiences have been observed in MMP-9 deficient mice (Van den Steen et al., 2002). However, young MMP-9 deficient mice are resistant to experimental autoimmune encephalomyelitis (Dubois et al., 1999). The gelatinases are also implicated in cardiovascular diseases. Loss of MMP-9 gene in atherosclerosis-prone mice reduced the growth of atherosclerotic lesions, and protected mice from the destruction of the atherosclerotic media implicating that MMP-9 is intimately involved in the pathogenesis of atherosclerosis (Luttun et al., 2004). The phenotype of MMP-2 deficient mice is relatively mild with minor defects in developmental angiogenesis and in the skeleton and joints (Corry et al., 2002; Itoh et al., 1998). However, tumor angiogenesis and tumor growth in the MMP2 deficient mice is highly reduced (Itoh et al., 1998). Significantly, MMP-2/MT1-MMP double knockout results in death of the mice immediately after birth with respiratory failure, abnormal blood vessels, and immature muscle fibers (Oh et al., 2004). In contrast, MMP2/MMP-9 knockout mice are viable (Baluk et al., 2004; Corry et al., 2004). These gelatinase double-knockout mice have been tested in a mycoplasma infection model in the airways. These gelatinase-deficient mice did not differ from their wild type littermates in the inflammatory response, except for that they could not induce gelatinase expression. The gelatinase-deficiency did not affect leukocyte influx into the airway lumen and lung mucosa, neither was the infection-associated microvascular remodelling affected by the lack of gelatinases (Baluk et al., 2004). However, in another inflammation model, although the accumulation of inflammatory cells in the lungs was not affected, a decreased number of inflammatory cells was found in the airway lumen of the MMP-9 and the double knockout mice due to a defect in the transepithelial chemokine gradient formation (Corry et al., 2004). It will be interesting to see the effect of the double knockout on tumor development and metastasis.

The gelatinases participate also in wound repair (Legrand et al., 1999; Mohan et al., 2002; Salonurmi et al., 2004) and are typically expressed from the beginning to the end of the healing process (Salo et al., 1994). Gelatinases and other MMPs are also able to participate in the regulation of apoptosis. MMP-9 has been observed to decrease cancer-cell apoptosis (Bergers et al., 2000), whereas developmental apoptosis is augmented ( $\mathrm{Vu}$ et al., 1998). MMP-2, or more specifically a C-terminal naturally occurring fragment of MMP-2 can induce apoptosis in tumor and endothelial cells (Bello et al., 2001). In addition, mice deficient in MMP-2, -3 or -9 show reduced hepatocyte apoptosis in a lethal hepatitis model (Wielockx et al., 2001).

\section{Structural features of matrix metalloproteinases}

MMPs can be grouped to eight classes, based on their domain structure (Figure 2). All MMPs contain a N-terminal predomain that is required for the correct secretion of these enzymes. The predomain is followed by a prodomain. The prodomain forms an essential contact with the catalytic zinc ion and maintains the latency of the MMPs (see below). The prodomain is followed by a catalytic domain, which contains the characteristic signatures for zinc-dependent metalloenzymes. The catalytic center of MMPs contains a zinc-binding HEBXHXBGBXHS motif, where $\mathrm{H}$ is histidine, $\mathrm{E}$ is glutamic acid, $\mathrm{B}$ is a bulky hydrophobic amino acid, $G$ is glycine, $X$ is variable amino acid and $S$ is serine. The serine 
can also be replaced by a threonine in a few MMPs, such as MMP-11 (Stocker et al., 1995). There is also an absolutely conserved methionine residue located on the opposite site of the zinc ion as compared to the HEBXHXBGBXHS motif. However, the role of this conserved methionine is unclear as serine or leucine mutants of this residue in MMP-2 show identical proteolytic activity towards various substrates (Butler et al., 2004). All MMPs except MMP7, MMP-23 and MMP-26 contain a hemopexin/vitronectin-like domain (Gomis-Ruth, 2004), which is linked to the catalytic domain by a short linker or a hinge region. The role of the hinge region in MMPs is unclear, although it has been reported that mutations in the MMP-8 hinge region affect autoproteolysis and substrate specificity (Knauper et al., 1997). MMP-9 has additionally a unique collagen V-like insertion between the catalytic domain and the C-terminal domain. The function of this insertion is unknown, but it contains most of the O-linked glycans of MMP-9 (Mattu et al., 2000; Rudd et al., 1999). The hemopexin/vitronectin-like C-terminal domain is responsible for multiple protein-protein interactions. It binds tissue inhibitors of matrix metalloproteinases (TIMPs), certain MMP substrates and is involved in the activation of some MMPs. The hemopexin-like domain also participates in the homodimerization of MMP-9 (Cha et al., 2002) and MT1-MMP (Lehti et al., 2002). Also heterodimers of MMP-9 and MMP-1 can form through the Cterminal domain interactions (Goldberg et al., 1992). Whereas MMP-7 and MMP-26 lack the hemopexin -like domain completely, MMP-23 has a cysteine- and proline-rich interleukin-1 type II receptor -like domain instead of a hemopexin-like domain. Furthermore, MMP-23 is bound to the cell surface through a unique N-terminal signal anchor (Pei et al., 2000).

\section{1) Minimal domain MMPs}

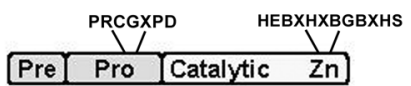

) Simple hemopexin-like domain-containing MMPs

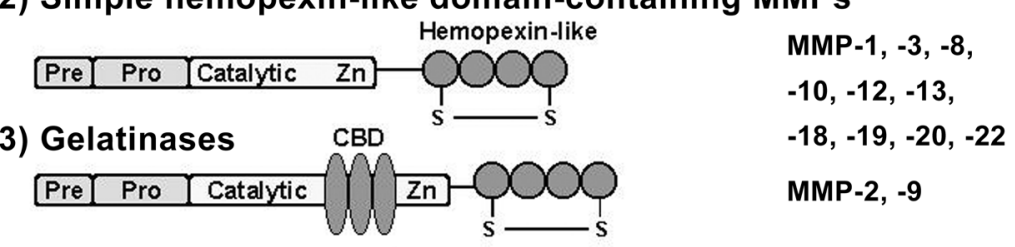

4) Furin-activated secreted MMPs

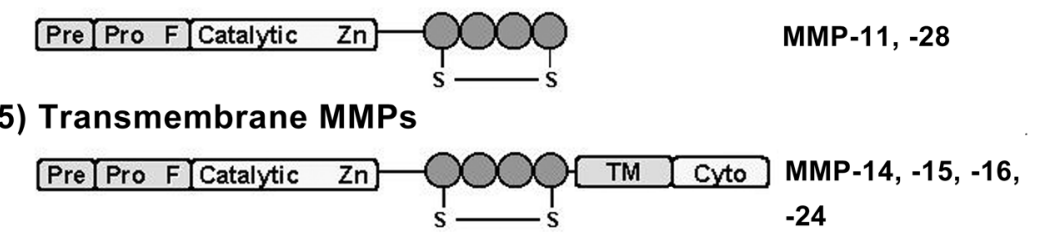

6) GPI-linked MMPs

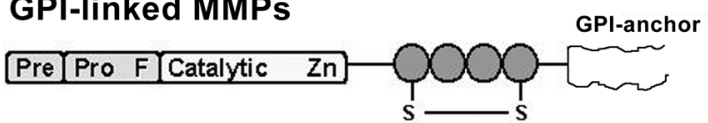

7) Vitronectin-like insert, linker-less MMPs

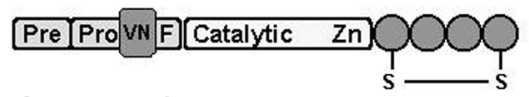

8) Cysteine/proline-rich IL-1 receptor-like domain MMPs

MMP-17, -25

MMP-21
Figure 2. Domain structure of MMPs. Pre, signal sequence; Pro, propeptide with the cysteine switch sequence; $\mathrm{Zn}$, zinc-ion binding site with the consensus sequence indicated; CBD, collagen/gelatin binding domain; $F$, furincleavage site; TM, transmembrane domain; Cyto, cytoplasmic domain; Vn, vitronectinlike insertion; IL-1R-like, interleukin-1 receptor like domain. Modified from Sternlicht and Werb (2001). 
Other extra domains that are not common to all members of MMPs include the collagenbinding domain (CBD) of gelatinases and the transmembrane domains of MT-MMPs. The CBD domain is composed of three fibronectin type II like repeats and is involved in binding of collagenous substrates and elastin (Steffensen et al., 1995), fatty acids (Berton et al., 2001) and thrombospondins (Bein and Simons, 2000). Although most of the MMPs are secreted proteins, six of them contain a transmembrane domain that is used to anchor these proteins on the cell surface. These MT-MMPs have a single pass transmembrane domain and a short cytoplasmic domain (MMP-14, -15, -16 and -24) or a glycophosphatidyl inositol (GPI) insertion signal (MMP-17 and -25).

The first complete MMP structure to be solved was that of proMMP-2 (Figure 3) (Morgunova et al., 1999). The proMMP-2 has also been crystallized with the tissue inhibitor of matrix metalloproteinase (TIMP)-2 (Morgunova et al., 2002). The structures of many other MMP domains have been solved and these provide a scaffold for the development of MMP-binding small molecules. For example, three different partial structures of MMP-9 with the level of $2.5 \AA$ resolution or better have been published. One is a C-terminally deleted proMMP-9 construct lacking the collagen V-like region and the hemopexin-like domain (Elkins et al., 2002). A catalytic domain of MMP-9 lacking the collagen-binding domain has been crystallized in the presence of a hydroxamate inhibitor (Rowsell et al., 2002). The C-terminal domain is the third resolved MMP-9 structure and this structure reveals the mechanism of MMP-9 dimerization (Cha et al., 2002). Together, these structures span the whole proMMP-9 except for the collagen $\mathrm{V}$-like region.

Figure 3. Structure of proMMP-2. The different domains as well as the individual fibronectin type II (Fnll) repeats are shown. ProMMP-9 differs from proMMP-2 primarily by having the collagen $\mathrm{V}$-like hinge region between the catalytic domain the C-terminal hemopexin/vitronectin-like domain. The effect of the collagen $\mathrm{V}$-like insertion to the overall structure of MMP-9 is not known. The proMMP-2 structure was generated with Swiss-PdbViewer v3.7 from the PDB entry 1CK7 (Morgunova et al., 1999).

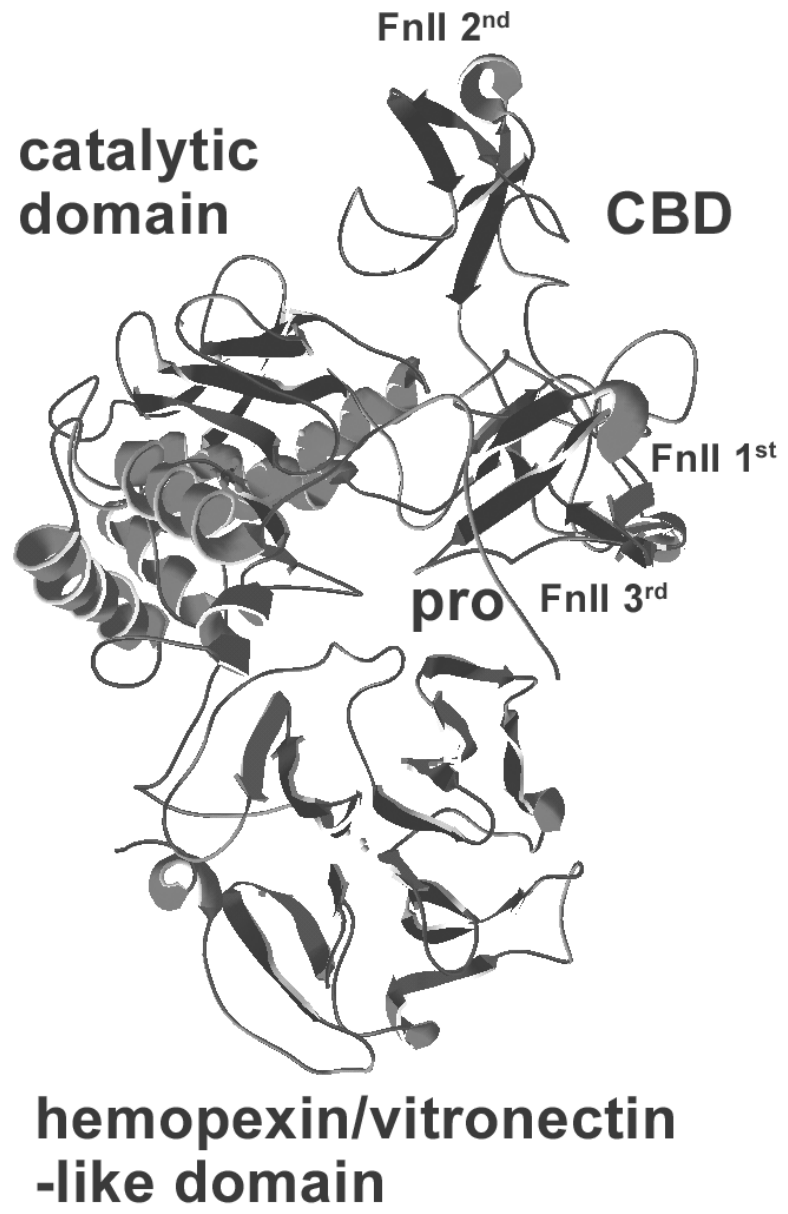




\section{Gelatinase substrates}

Gelatinase substrates include a wide variety of proteins including ECM proteins, proteinases, proteinase inhibitors, blood clotting factors, chemotactic molecules, latent growth factors and growth factor binding proteins, cell surface receptors, adhesion molecules and even intracellular substrates (Table 1). However, the relevance of these events in vivo is unclear at present. The concensus cleavage sequences of both MMP-2 and MMP-9 have been mapped with the substrate phage (Chen et al., 2002; Kridel et al., 2001). The substrate specificities of MMP-2 and MMP-9 are similar but not identical. The most notable difference is the ability of MMP-2 to degrade native type I collagen. The difference in the substrate specificity of gelatinases has been attributed to the S2 subsite in the catalytic site, where MMP-9 contains an aspartic acid, and MMP-2 a glutamic acid (Chen et al., 2003). The catalytic domains of MMP-2 and MMP-9 also differ in their S1' binding pockets (Elkins et al., 2002; Rowsell et al., 2002). These minor differences in the catalytic domain have functional consequences in the substrate selectivity of the gelatinases. The amino acid residue fitting to the S2 subsite in the gelatinase substrates appears to be a major selectivity determinant based on the cleavage sequences obtained with the substrate-phage (Table 2). Indeed, some substrates show over 200-fold selectivity towards MMP-2 (Chen et al., 2002; Kridel et al., 2001).

Table 1. Gelatinase substrates

\begin{tabular}{|c|c|c|c|}
\hline Matrix substrates & MMP-2 & MMP-9 & Reference \\
\hline Denatured collagens (gelatins) & yes & yes & (Morodomi et al., 1992; Okada et al., 1990) \\
\hline Native collagen type: I & yes & no & (Aimes and Quigley, 1995) \\
\hline III & yes & no & (Berton et al., 2000) \\
\hline IV & yes & yes & (Morodomi et al., 1992) \\
\hline V & yes & yes & (Morodomi et al., 1992) \\
\hline VII & yes & & (Seltzer et al., 1989) \\
\hline $\mathrm{X}$ & yes & & (Cole et al., 1993) \\
\hline $\mathrm{XI}$ & yes & & (Smith et al., 1991) \\
\hline XVIII & & yes & (Ferreras et al., 2000) \\
\hline Aggrecan & yes & yes & (Fosang et al., 1992) \\
\hline BM-40/SPARC/ Osteonectin & yes & yes & (Sasaki et al., 1997) \\
\hline Brevican & yes & no & (Nakamura et al., 2000) \\
\hline Decorin & yes & no & (Imai et al., 1997) \\
\hline Elastin & yes & yes & (Murphy et al., 1991) \\
\hline Entactin/nidogen & no & yes & (Mayer et al., 1993; Sires et al., 1993) \\
\hline Fibrillin & yes & yes & (Ashworth et al., 1999) \\
\hline Fibrin & & yes & (Lelongt et al., 2001) \\
\hline Fibrinogen & yes & yes & (Bini et al., 1996; Lelongt et al., 2001) \\
\hline Fibronectin & yes & no & (Okada et al., 1990) \\
\hline Laminin & yes & yes & $\begin{array}{l}\text { (Giannelli et al., 1997; Morodomi et al., } \\
\text { 1992) }\end{array}$ \\
\hline Link protein & yes & yes & (Nguyen et al., 1993) \\
\hline NG2 proteoglycan & & yes & (Larsen et al., 2003) \\
\hline Neurocan & yes & & (Turk et al., 2001) \\
\hline Tenascin & yes & no & (Siri et al., 1995) \\
\hline Vitronectin & yes & yes & (Imai et al., 1995) \\
\hline
\end{tabular}


Table 1. continued

\begin{tabular}{|c|c|c|c|}
\hline Bioactive substrates & MMP-2 & MMP-9 & Reference \\
\hline a1-proteinase inhibitor & & yes & (Liu et al., 2000) \\
\hline a2-macroglobulin & yes & yes & (Arbelaez et al., 1997) \\
\hline aB-crystallin & & yes & (Starckx et al., 2003) \\
\hline Amyloid protein precursor & yes & & (LePage et al., 1995) \\
\hline Big endothelin-1 & yes & yes & (Fernandez-Patron et al., 2002) \\
\hline $\begin{array}{l}\text { Calcitonin gene-related peptide } \\
\text { (CGRP) }\end{array}$ & yes & & (Fernandez-Patron et al., 2000) \\
\hline Complement protein $\mathrm{C} 1 \mathrm{q}$ & yes & yes & (Ruiz et al., 1999) \\
\hline $\begin{array}{l}\text { Connective tissue-activating peptide- } \\
\text { III (CTAP-III) }\end{array}$ & & yes & (Van den Steen et al., 2000) \\
\hline Eph B1 tyrosine kinase receptor & yes & no & (Chen et al., 2002) \\
\hline $\begin{array}{l}\text { Epithelial-cell derived neutrophil } \\
\text { activating peptide-78/CXCL5 (ENA- } \\
\text { 78) }\end{array}$ & & yes & (Van Den Steen et al., 2003) \\
\hline $\begin{array}{l}\text { Fibroblast growth factor receptor } \\
\text { (FGFR) }-1\end{array}$ & yes & no & (Levi et al., 1996) \\
\hline Galectin-3 & yes & yes & (Ochieng et al., 1994) \\
\hline $\begin{array}{l}\text { Granulocyte } \\
\text { chemotactic protein-2 } \\
\text { /CXCL6 (GCP-2) }\end{array}$ & & yes & (Van Den Steen et al., 2003) \\
\hline Growth-regulated oncogene (GRO)- $\alpha$ & & yes & (Van den Steen et al., 2000) \\
\hline Insulin & & yes & (Descamps et al., 2003) \\
\hline $\begin{array}{l}\text { Insulin-like growth factor binding } \\
\text { proteins (IGFBP) }\end{array}$ & yes & yes & $\begin{array}{l}\text { (Fowlkes et al., 1994; Manes et al., 1999; } \\
\text { Thrailkill et al., 1995) }\end{array}$ \\
\hline $\begin{array}{l}\text { Intercellular adhesion } \\
\text { molecule (ICAM)-1 }\end{array}$ & & yes & (Fiore et al., 2002) \\
\hline Interferon (IFN)- $\beta$ & & yes & (Nelissen et al., 2003) \\
\hline $\begin{array}{l}\text { Interferon-inducible protein-10 (IP- } \\
\text { 10/CXCL-10) }\end{array}$ & & yes & (Van den Steen et al., 2003) \\
\hline Interleukin receptor IL-2R $\alpha$ & no & yes & (Sheu et al., 2001) \\
\hline KiSS-1 protein/metastin & yes & yes & (Takino et al., 2003) \\
\hline Kit-ligand & & yes & (Heissig et al., 2002) \\
\hline $\begin{array}{l}\text { Monocyte chemoattractant protein } \\
\text { MCP-3 }\end{array}$ & yes & no & (McQuibban et al., 2002) \\
\hline $\begin{array}{l}\text { Monokine induced by interferon IFN-Y } \\
\text { (MIG/CXCL-9) }\end{array}$ & & yes & (Van den Steen et al., 2003) \\
\hline Myelin basic protein & yes & yes & (Chandler et al., 1995) \\
\hline Myosin heavy chain & yes & yes & (Rouet-Benzineb et al., 1999) \\
\hline Plasminogen & yes & yes & $\begin{array}{l}\text { (O'Reilly et al., 1999; Patterson and Sang, } \\
\text { 1997) }\end{array}$ \\
\hline Platelet factor (PF)-4 & & yes & (Van den Steen et al., 2000) \\
\hline Poly (ADP-ribose) polymerase (PARP) & yes & & (Kwan et al., 2004) \\
\hline Pregnancy zone protein & yes & yes & (Arbelaez et al., 1997) \\
\hline Pro-IL-1 $\beta$ & yes & yes & (Schonbeck et al., 1998) \\
\hline Pro-IL-8 & & yes & (Van den Steen et al., 2000) \\
\hline MMP-1 (trypsin-activated) & yes & & (Crabbe et al., 1994) \\
\hline Pro-MMP-2 & yes & & (Crabbe et al., 1993) \\
\hline Pro-MMP-9 & yes & yes & (Fridman et al., 1995; Ray et al., 2003) \\
\hline Pro-MMP-13 & yes & & (Knauper et al., 1996) \\
\hline Pro-TGF- $\beta 1$ & yes & yes & (Yu and Stamenkovic, 2000) \\
\hline Pro-TNF- $\alpha$ & yes & yes & (Gearing et al., 1994) \\
\hline Pro-urokinase & yes & & (Prager et al., 2003) \\
\hline Stromal cell derived factor (SDF)-1 & yes & yes & (McQuibban et al., 2001) \\
\hline Substance P & & yes & (Backstrom and Tokes, 1995) \\
\hline Troponin & yes & & (Wang et al., 2002) \\
\hline Urokinase receptor & yes & no & (Andolfo et al., 2002) \\
\hline
\end{tabular}


Gelatinase binding to native collagens and gelatin occurs primarily via the CBD (Allan et al., 1995), whereas other MMPs, eg. MMP-3 utilizes the C-terminal domain for collagen binding (Allan et al., 1991). However, gelatin binds also to the C-terminal domain of the gelatinases (Collier et al., 2001; Roeb et al., 2002). It has been shown that binding of type I collagen to the C-terminal domain and the catalytic domain of MMP-2 is sufficient for collagenolysis, whereas subsequent gelatinolysis requires the participation of the CBD (Patterson et al., 2001). The C-terminal domain of gelatinases may also bind noncollagenous substrates (McQuibban et al., 2000). The residues contributing to the gelatin binding in the CBD have been identified by site-directed mutagenesis, and are located in the second fibronectin type II module of both gelatinases (Collier et al., 1992; Tordai and Patthy, 1999). These gelatin binding residues in MMP-2 have been thoroughly examined by NMR using gelatin-mimicking (proline-proline-glycine) ${ }_{n}$ peptides (Briknarova et al., 2001; Briknarova et al., 1999; Gehrmann et al., 2002). Peptides binding to the recombinant CBD and the individual fibronectin type repeats of MMP-2 have been isolated using phage display, but they do not show any significant similarity to sequences found in collagens or other potential substrates (Trexler et al., 2003).

The three fibronectin type II repeats form a three-pronged fishhook -like structure in proMMP-2 (Morgunova et al., 1999), and this conformation may be needed for the unwinding and complete degradation of the triple helical collagens (Overall, 2002). In the proMMP-2 structure, the prodomain peptide PIIKFPGDVA interacts intramolecularly with the putative gelatin-binding site of the third fibronectin type II repeat via contacts that involve propeptide amino acid residues $\mathrm{Ile}^{35}, \mathrm{Phe}^{37}$, and $\mathrm{Asp}^{40}$ (Morgunova et al., 1999). This binding may represent an additional mechanism in maintaining the latency of the progelatinases. It is also of interest to note that the relative affinities of the substrates to the gelatinases may vary depending on the activation status of the enzyme. ProMMP-9 binds type I collagen with a higher affinity than active MMP-9, whereas the opposite is true for type IV collagen recognition (Allan et al., 1995). The biological significance of these differences in the affinities is unclear at present.

Table 2. The consensus cleavage sites of the gelatinases

\begin{tabular}{|c|c|c|c|c|c|}
\hline & \multirow{2}{*}{$\begin{array}{l}\text { Consensus } \\
\text { cleavage site }^{a}\end{array}$} & \multirow{2}{*}{$\begin{array}{l}\text { Example } \\
\text { peptide }\end{array}$} & \multicolumn{3}{|c|}{ Selectivity } \\
\hline & & & vs.MMP-9 & vs.MMP-7 & vs.MMP-13 \\
\hline MMP-2, group I & $\mathrm{PXX}^{\prime} \mathrm{X}_{\mathrm{Hy}}$ & AKPRA'LTA & 2 & 21 & 14 \\
\hline II & $\mathrm{I} / \mathrm{LX} X^{\prime} X_{\mathrm{Hy}}$ & LRLA'AITA & 14 & 6 & 13 \\
\hline III & $\mathrm{X}_{\mathrm{Hy}} \mathrm{SX} X^{\prime} \mathrm{L}$ & NRYSS'LTA & 40 & 84 & 24 \\
\hline IV & $H X X^{\prime} X_{H y}$ & HMHAA'LTA & 100 & n.d. & n.d. \\
\hline MMP-9, group I & $\mathrm{PR}(\mathrm{S} / \mathrm{T})^{\prime} \mathrm{X}_{\mathrm{Hy}}(\mathrm{S} / \mathrm{T})$ & KGPRQ'ITA & n.a. & 14 & 12 \\
\hline II & XXG'L(K/R)X & GSG'LKA & n.a. & 1 & 0.3 \\
\hline III & $\mathrm{XRR}^{\prime} \mathrm{X}_{\mathrm{Hy}}(\mathrm{I} / \mathrm{L}) \mathrm{X}$ & GRR'LLSR & n.a. & n.d. & n.d. \\
\hline
\end{tabular}

\section{Regulation of MMP activity}

In order to avoid unwanted tissue damage it is crucial to accurately control the protease activity. For this reason, protease activity is typically regulated at multiple levels including transcription, secretion, activation, and by the action of proteinase inhibitors. MMPs including the gelatinases are no exception in this respect. 


\section{Expression and secretion of gelatinases and other MMPs}

A major difference between MMP-2 and MMP-9 is their differential regulation of expression (Huhtala et al., 1991). Typically, MMP-2 is rather constitutively expressed with only modest up or downregulation under various conditions (Birkedal-Hansen et al., 1993). Instead, MMP-9 expression is highly inducible and under the control of growth factors, chemokines and other stimulatory signals (Hipps et al., 1991). These differences can be traced to the promoter elements of the gelatinases (Sato and Seiki, 1993; Van den Steen et al., 2002; Westermarck and Kähäri, 1999). The promoter of MMP-9 is similar to most other MMPs, whereas MMP-2 promoter lacks many of the inducible promoter elements such as binding sites for the AP-1 and ETS transcription factors (Westermarck and Kähäri, 1999). The differential regulation of MMP-2 and MMP-9 expression is evident in cells treated with protein kinase $\mathrm{C}$ activators such as phorbol esters. Whereas the expression of MMP-9 is stimulated several fold, MMP-2 expression is slightly downregulated (Huhtala et al., 1991). Cytokines and growth factors that activate MMP-9 expression typically act via the mitogenactivated protein kinase (MAPK) pathway, which includes the ERK 1/2, JNK/SAPK 1/2 and p38 proteins (Westermarck and Kahari, 1999). These inducers include epidermalgrowth factor (EGF), platelet-derived growth factor (PDGF), hepatocyte growth factor/scatter factor (HGF/SF), basic fibroblast growth factor (bFGF), transforming growth factor (TGF)- $\alpha$ and $\beta$, amphiregulin, tumor necrosis factor (TNF)- $\alpha$, interleukin (IL)- $1 \alpha$ and $\beta$, interferon (IFN)- $\alpha$ and $\gamma$ (Van den Steen et al., 2002). Due to the highly inducible nature of MMP-9 by growth factors and cytokines, MMP-9 promoter activity can be detected in all invasive tumors in mice carrying a $\beta$-galactosidase gene under the control of MMP-9 promoter (Kupferman et al., 2000).

MMP-1, -2, -3 and MT1-MMP, but not MMP-9 expression, can also be stimulated by the extracellular matrix metalloproteinase inducer (EMMPRIN) (Caudroy et al., 2002). EMMRPIN is a cell surface glycoprotein belonging to the immunoglobulin superfamily. In addition to the MMP expression stimulating activity, EMMPRIN acts as a cell surface receptor for MMP-1 (Guo et al., 2000). Other proteins that are able to regulate the activity of MMPs include the three small integrin-binding ligand N-linked glycoproteins (SIBLINGs); bone sialoprotein, osteopontin and dentin matrix protein-1. Bone sialoprotein specifically binds MMP-2, while osteopontin binds MMP-3, and dentin matrix protein-1 binds MMP-9. Interestingly, binding of the SIBLINGS to the MMPs activates the proenzymes and reduces their susceptibility to the MMP-inhibitors (Fedarko et al., 2004).

Besides the growth factor and chemokine-induced signals, integrin and extracellular matrix -mediated signals regulate the expression of the gelatinases and other MMPs. It was initially observed that antibodies blocking $\alpha_{5} \beta_{1}$ integrin-mediated adhesion induced MMP expression in fibroblasts (Werb et al., 1989). Later studies revealed that integrin-mediated signals are general regulators of MMP expression, as $\alpha_{2} \beta_{1}$ integrin regulates MMP-1 expression (Riikonen et al., 1995; Dumin et al., 2001), antibodies to $\alpha_{3} \beta_{1}$ integrin-tetraspanin complexes induce MMP-2 expression (Sugiura and Berditchevski, 1999), and $\alpha_{M} \beta_{2}$ and $\alpha_{3} \beta_{1}$ integrin ligation stimulates MMP-9 expression (Wize et al., 1998; Larjava et al., 1993). The effects on MMP expression are very specific. For example, $\alpha_{4} \beta_{1}$ integrin ligation by vascular cell adhesion molecule-1 in T cells induces only MMP-2 and not MMP-9, but ligation of the same integrin to a fibronectin-derived CS-1 peptide stimulates the expression of both gelatinases (Yakubenko et al., 2000). Although speculative, it seems that the integrin-matrix interactions induce selective expression of those MMPs that are the most suitable for the modification of the underlying matrix. 
Leukocytes, which have to rapidly adhere to the blood vessel endothelium and extravasate into tissues, have developed an additional mechanism to control gelatinase activity. These cells have large amounts of protease-containing granules, which are rapidly delivered to the cell surface and excreted to the extracellular space after leukocyte activation. Due to a high MMP-9 content, one of these granule types is called as gelatinase granules (Borregaard, 1997; Cowland and Borregaard, 1999). Adherent cell types do not contain storage granules of gelatinases and are dependent on direct secretion of the newly synthesized enzymes. In endothelial and tumor cells, the gelatinases are secreted in specific transport vesicles containing also other proteins such as integrins and components of the plasminogen activation system (Dolo et al., 1999; Ginestra et al., 1997; Taraboletti et al., 2002). Integrins appear to be required for the delivery of these vesicles. For example, MMP-9 was not secreted into the cell culture medium of keratinocytes in the absence of $\alpha_{3} \beta_{1}$ (DiPersio et al., 2000). Similarly, antisense expression of $\alpha_{V}$ integrin reduced MMP-9 expression in EpsteinBarr virus infected B lymphocytes (Huang et al., 2000) and expression of $\alpha_{V} \beta_{6}$ in colon carcinoma increased secreted MMP-9 (Agrez et al., 1999; Niu et al., 1998). Also urokinaseplasminogen activator receptor (UPAR) regulates MMP-9 secretion. Antisense expression of uPAR abrogates MMP-9 expression, but not MMP-2 or integrin expression (Aguirre Ghiso et al., 1999; Ahmed et al., 2003). An inverse correlation of integrin expression and MMP expression has been observed in HT1080 fibrosarcoma cells where overexpression of MT1MMP significantly reduced and antisense expression increased $\alpha_{\mathrm{V}} \beta_{3}$ integrin expression (Monea et al., 2002).

\section{Proenzyme activation}

The gelatinases are secreted as proenzymes, and they need to be activated for full catalytic activity. The activation of the gelatinases and other soluble MMPs occurs on the cell surface or in the extracellular milieu, whereas MT-MMPs may also be activated intracellularly by furin-like proprotein convertases (Sato et al., 1996; Yana and Weiss, 2000; Zucker et al., 2003). In general, proteases can be activated via multiple mechanisms and commonly a reciprocal activation pattern is observed, i.e., an activated protease can activate the zymogen of its activator (Ellis, 2003). Hence, it is highly difficult to define the initiator protease of the activation cascades and also how the first enzyme in the cascade is activated. One possibility is that some of the proenzymes are sufficiently active for the initial cleavage.

The MMPs become catalytically active when the propeptide of an MMP is cleaved or the conformation of the propeptide is disrupted. The "cysteine switch" principle is essential for understanding the MMP activation process (Figure 4). MMPs contain a conserved peptide sequence PRCGXPD in the prodomain. The cysteine residue coordinates with the catalytic zinc ion and blocks the entry of a catalytically essential water molecule to the active site (Van Wart and Birkedal-Hansen, 1990). The various activation mechanisms of MMPs all disrupt the interaction of the critical cysteine with the catalytic zinc atom either by removing the prodomain or by modifying the cysteine residue in the prodomain. This allows the entrance of a water molecule in the catalytic site and results in the formation of an active catalytic centre. The cysteine switch mechanism explains why proteases, conformational perturbants such as heat, denaturants or substrate binding, heavy metals and organomercurials, oxidants and alkylating agents are able to activate MMPs (Van Wart and Birkedal-Hansen, 1990; Visse and Nagase, 2003). 

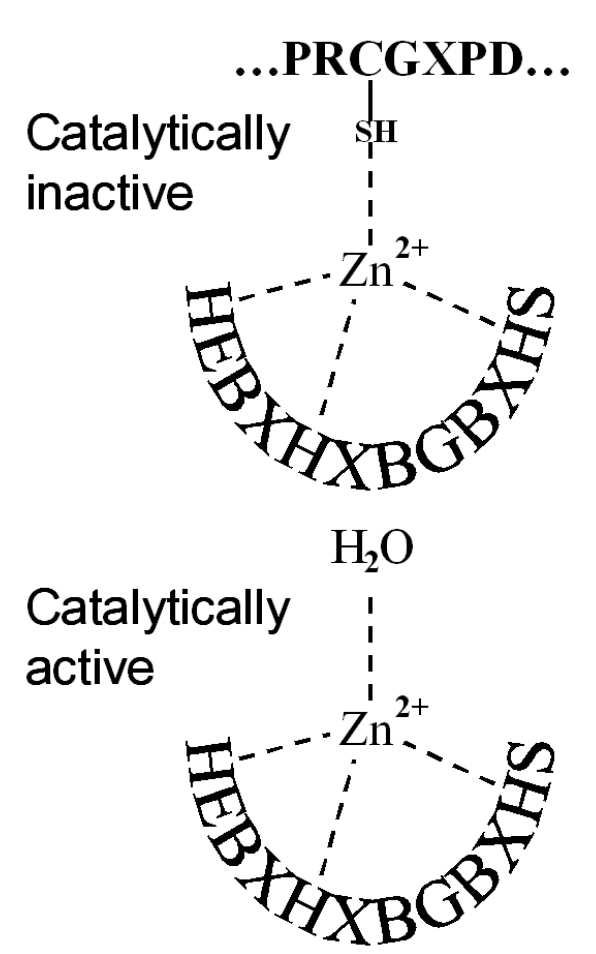

Figure 4. The architecture of the catalytic site of MMPs. The thiol group from the cysteine side chain in the conserved PRCGXPD sequence present in the propeptide coordinates with the catalytic zinc ion in the latent MMPs. During MMP activation a water molecule replaces the thiol group resulting in a catalytically active enzyme.

The main activation route of MMP-2 on the cell surface is by the formation of a molecular complex containing MMP-2, MT1-MMP and TIMP-2 (Murphy and Crabbe, 1995; Strongin et al., 1995; Lehti et al., 1998). The N-terminal domain of TIMP-2 binds to and inhibits MT1-MMP, whereas the C-terminal domain of the same TIMP-2 molecule binds the hemopexin-like domain of MMP-2 forming a ternary complex. An adjacent TIMP-free MT1-MMP subsequently cleaves the MMP-2 to the intermediate 64-kDa form by cleaving the $\mathrm{Asn}^{37}$-Leu ${ }^{38}$ bond located in a readily accessible bait region between the first and the second $\alpha$ helix of the prodomain. This intermediate form is then processed to the fully mature $62-\mathrm{kDa}$ form through cleavage of the $\mathrm{Asn}^{80}-\mathrm{Tyr}^{81}$ bond by an already active MMP-2 molecule (Murphy and Crabbe, 1995; Strongin et al., 1995). Only the active MT1-MMP binds MMP-2 on the cell surface, thus regulation of MT1-MMP activation is an important control point to regulate MMP-2 activity (Lehti et al., 1998). Although TIMP-2 is normally required for the MMP-2 activation, higher TIMP-2 levels lead to inhibition of MMP-2 activation. On the other hand, soluble MT1-MMP activates MMP-2 with a high efficiency in the absence of TIMP-2 (Pei and Weiss, 1996). Integrins are also involved in the activation process directly or indirectly as $\alpha_{V} \beta_{3}$ and $\beta_{1}$ integrin activating antibodies modulate MMP-2 activation (Yan et al., 2000). The direct involvement is supported by the finding that the intermediate active MMP-2 is capable of $\alpha_{\mathrm{V}} \beta_{3}$ integrin binding, and this interaction could thus affect MMP-2 activation (Brooks et al., 1996; Deryugina et al., 2001). Alternatively, integrin-mediated signals may lead to the increase in MT1-MMP expression and activity and thus contribute to the MMP-2 activation. Endothelial cells cultured in a three-dimensional (3D)-collagen type I matrix express MT1-MMP with concomitant activation of MMP-2 whereas only a minor fraction of MMP-2 is activated in a 2D-collagen culture (Haas et al., 1998). In addition, HT1080 cells cultured on fibronectin, but not on laminin-1 show increased activation of MMP-2. The activation involves integrin activity as immobilized antibodies to the fibronectin receptor $\alpha_{5} \beta_{1}$ similarly induce MMP-2 activation (Stanton et al., 1998).

MMP-2 can also be activated by MMP-1 and MMP-7, and the activated anti-coagulant protein C (Nguyen et al., 2000; Sang et al., 1996), but not with MMP-3, trypsin, 
chymotrypsin, plasmin, plasma kallikrein, thrombin, neutrophil elastase, cathepsin $\mathrm{G}$ or thermolysin (Okada et al., 1990). However, proteases that do not directly activate MMP-2 can do so by activating MT1-MMP (Shamamian et al., 2000). Cell surface activation of MMP-2 can also occur through a plasminogen-mediated mechanism, which requires the presence of MT1-MMP, but not MT1-MMP activity (Monea et al., 2002). MMP-2 activation can also be induced with the lectin concanavalin A (Overall and Sodek, 1990). The effect is likely mediated by inhibition of MT1-MMP endocytosis from the cell surface (Jiang et al., 2001). Intracellular activation of MMP-2 has also been observed, but the mechanism has not been elucidated (Lee et al., 1997).

The physiological activation mechanism(s) of MMP-9 still remain(s) poorly understood. Plasminogen/MMP-3 mediated activation (Ramos-DeSimone et al., 1999) and activation by trypsin-2 (Sorsa et al., 1997) appear to be the most potent activators of MMP-9. Plasminogen/MMP-3 mediated activation involves activation of plasminogen to plasmin by urokinase plasminogen activator (UPA). UPA is bound to its cell surface receptor UPAR and cleaves plasminogen. Plasmin is an efficient activator of proMMP-3, which then activates MMP-9 by sequential cleavage of the $\mathrm{Glu}^{40}-\mathrm{Met}^{41}$ and $\mathrm{Arg}^{87}-\mathrm{Phe}^{88}$ bonds (Murphy and Crabbe, 1995). In vivo, MMP-3 deficiency does not impair MMP-9 activation (Lijnen et al., 1998), however other MMPs may complement MMP-9 activation in these mice. MMP-9 can also be directly activated by plasmin but this is a relatively inefficient process. However, the plasmin-mediated activation is potentiated on the cell surface (Mazzieri et al., 1997). Plasmin clearly contributes to in vivo activation of MMP-9 as the uPA-deficient mice show reduced activation of MMP-9, as well as that of MMP-3, -12 and -13 (Carmeliet et al., 1997). In vitro, MMP-9 can also be activated using MMP-2 (Fridman et al., 1995), MMP-3 (Ogata et al., 1992), MMP-7 (von Bredow et al., 1998), MMP-10 (Nakamura et al., 1998), MMP-13 (Knauper et al., 1997) and MMP-26 (Zhao et al., 2003), which all yield the fully active $82 \mathrm{kDa}$ MMP-9.

The human tumor-associated trypsin-2 is an efficient activator of MMP-9 (Sorsa et al., 1997). MMP-9 could be activated at a 1:1000 trypsin to proMMP-9 ratio. Similarly to the MMP-3 mediated activation, the propeptide of MMP-9 was completely removed by cleavage of the $\mathrm{Arg}^{87}-\mathrm{Phe}^{88}$ bond (Sorsa et al., 1997). Recently, it was reported that MMP-9 can be activated by MT1-MMP/MMP-2 and MMP-3 on the cell membranes more efficiently than in solution (Toth et al., 2003). Chymotrypsin-like proteinase in human skin (Han et al., 2002) and mast cell derived $\alpha$ and $\beta$ chymase (Coussens et al., 1999; Fang et al., 1997) have also been reported to activate MMP-9. More complexity comes from the finding that the proMMP-9 displays significant catalytic activity in the presence of the propeptide (Bannikov et al., 2002). Substrate binding appears thus to be sufficient to trigger the cysteine switch and provides one possible solution to the dilemma that active MMP-9 is often not found in the tissues despite the catalytic activity of MMP-9. Neutrophils are also able to activate MMP-9 via an oxidative mechanism (Peppin and Weiss, 1986). Additionally, it has been observed that MMP-9 can be activated by S-nitrosylation ( $\mathrm{Gu}$ et al., 2002). Both gelatinases can be activated via free radical-generating 2- and 4hydroxyestradiols (Paquette et al., 2003). Organomercurials such as aminophenylmercuric acetate (APMA) are commonly used as MMP activators. With APMA, the propeptide of MMP-9 is not completely removed. Furthermore, a prolonged incubation with APMA results in the autolytic processing of the C-terminal domain with three successive cleavages (Triebel et al., 1992). 


\section{GELATINASE INHIBITORS}

\section{Naturally occuring gelatinase inhibitors}

Both naturally occurring and synthetic MMP inhibitors have been identified and characterized. Some of them show significant selectivity towards the gelatinases (Table 3). The physiological inhibitors of gelatinases include $\alpha 2$-macroglobulin and the tissue inhibitors of MMPs (TIMPs). $\alpha 2$-macroglobulin is an abundant plasma protein and effectively inhibits the activity of most proteinases, including the MMPs (Sottrup-Jensen and Birkedal-Hansen, 1989). Binding to $\alpha 2$-macroglobulin is an efficient indicator of MMP activation status as only the activated enzymes bind it (Morodomi et al., 1992). The a2macroglobulin may play an important role in the endocytic removal of proteolytic enzymes (Moestrup et al., 1993). The inhibitor-MMP complexes are internalized into cells via the low-density lipoprotein receptor-related protein (LRP) and are eventually degraded. However, MMP-9 can also be internalized as a monomer or TIMP-1 complex and MMP-2 with thrombospondin-2 apparently in the absence of $\alpha 2$-macroglobulin (Hahn-Dantona et al., 2001; Yang et al., 2001).

TIMPs are relatively small, cysteine rich proteins. They form high-affinity 1:1 complexes with the MMPs. TIMPs-1, 2, 3 and 4 vary in tissue specific expression and their ability to inhibit various MMPs (Egeblad and Werb, 2002). For example, TIMP-1 inhibits MMP-9 with a high affinity, whereas TIMP-2 inhibits MMP-2 (O'Connell et al., 1994; Olson et al., 1997). TIMPs also inhibit the activity of other metalloproteinases, namely members of the ADAM (a disintegrin and metalloproteinase) family (Amour et al., 2000; Amour et al., 1998; Egeblad and Werb, 2002). Studies with TIMP-2 knockout mice indicate that the dominant function of TIMP-2 in vivo is the activation of proMMP-2 (Wang et al., 2000). The crystal structure of proMMP-2 with TIMP-2 reveals the structural basis for this interaction required for MMP-2 activation. The C-terminal hemopexin-like domain of MMP-2 interacts with the C-terminal domain of TIMP-2, whereas the catalytic domain of MMP-2 and the MMP inhibitory N-terminal domain of TIMP do not form contacts in this structure (Morgunova et al., 2002). The MMP-2/TIMP-2 complex also reveals why TIMP-1 does not interact with MMP-2. This is because TIMP-1 lacks the critical C-terminal MMP-2 interacting residues present in TIMP-2 (Morgunova et al., 2002). Despite the MMPinhibitory activity of TIMPs, several studies have showed that a high level of TIMP-1 or -2 correlate with a poor prognosis in many types of cancer (Fong et al., 1996; Murashige et al., 1996; Ree et al., 1997; Yoshizaki et al., 2001). It is not known, whether this is due to an attempt to compensate for the increased MMP levels or an independent cause as TIMPs do have other functions independent on MMP inhibition. Under some conditions, the TIMPs can inhibit tumor-cell apoptosis as well as promote cell growth and angiogenesis. For example, TIMP-4 can upregulate an anti-apoptotic protein Bcl- $\mathrm{X}_{\mathrm{L}}$ and stimulate mammary tumorigenesis (Jiang et al., 2001). The growth promoting activities of TIMPs are not well understood, but are observed in many cell types and appear to be independent of MMPinhibitory activity (Nemeth et al., 1996). TIMPs have also MMP-independent cancerinhibiting functions. TIMP-2 may directly inhibit endothelial cell proliferation and angiogenesis by acting through $\alpha_{3} \beta_{1}$ integrin and causing a decrease in tyrosine phosphatase activity associated with this integrin (Seo et al., 2003). TIMP-3 can directly inhibit angiogenesis through blockage of VEGF binding to the VEGF receptor-2 (Qi et al., 2003). An interesting observation is that TIMP-1, in addition to the extracellular milieu, can also be found in the nucleus (Zhao et al., 1998), and may even be specifically translocated there from the cell membrane (Ritter et al., 1999). Recently, also MMP-2 was found to be present 
in the nucleus, and a pro-apoptotic nuclear protein, poly (ADP-ribose) polymerase was cleaved by MMP-2 in vitro suggesting that MMP-2 could partially substitute for caspases in the apoptotic cascade (Kwan et al., 2004).

Table 3. Inhibitors and negative regulators of gelatinases

\begin{tabular}{|c|c|c|c|}
\hline Inhibitor & $\begin{array}{l}\text { Mechanism of } \\
\text { action }\end{array}$ & Other targets $^{a}$ & Reference \\
\hline TIMP-1 & catalytic activity & $\begin{array}{l}\text { Most MMPs, ADAM-10, ADAMTS- } \\
4\end{array}$ & (Egeblad and Werb, 2002) \\
\hline TIMP-2 & catalytic activity & Most MMPs, ADAMTS-4 & (Egeblad and Werb, 2002) \\
\hline TIMP-3 & catalytic activity & $\begin{array}{l}\text { Most MMPs, ADAM-10, }-12,-17 \text {, } \\
\text { ADAMTS-4, }-5\end{array}$ & (Egeblad and Werb, 2002) \\
\hline TIMP-4 & catalytic activity & most MMPs, ADAMTS-4 (partly) & (Egeblad and Werb, 2002) \\
\hline a2-macroglobulin & $\begin{array}{l}\text { catalytic activity, } \\
\text { clearance }\end{array}$ & most proteases & (Morodomi et al., 1992) \\
\hline $\begin{array}{l}\text { Procollagen C- } \\
\text { terminal proteinase } \\
\text { enhancer (PCPE) }\end{array}$ & catalytic activity & & (Mott et al., 2000) \\
\hline $\begin{array}{l}\text { Tissue factor } \\
\text { pathway inhibitor-2 }\end{array}$ & $\begin{array}{l}\text { catalytic activity, } \\
\text { activation }\end{array}$ & serine proteases, other MMPs & (Herman et al., 2001) \\
\hline Endostatin & $\begin{array}{l}\text { inhibition of } \\
\text { activation, catalytic } \\
\text { activity }\end{array}$ & MT1-MMP & (Kim et al., 2000) \\
\hline RECK & catalytic activity & MT1-MMP & (Takahashi et al., 1998) \\
\hline Thrombospondins & $\begin{array}{l}\text { inhibition of } \\
\text { activation }\end{array}$ & & $\begin{array}{l}\text { (Rodriguez-Manzaneque } e t \\
\text { al., 2001) }\end{array}$ \\
\hline Neovastat & catalytic activity & $\begin{array}{l}\text { VEGF, induction of endothelial cell } \\
\text { apoptosis }\end{array}$ & (Dupont et al., 1998) \\
\hline Matlystatin & catalytic activity & & (Tanzawa et al., 1992) \\
\hline Aspirin & $\begin{array}{l}\text { reduction of } \\
\text { expression }\end{array}$ & Cyclooxygenases & (Jiang et al., 2001) \\
\hline $\begin{array}{l}\text { Epigallocatechin-3- } \\
\text { gallate }\end{array}$ & $\begin{array}{l}\text { inhibition of } \\
\text { activation, catalytic } \\
\text { activity, reduction of } \\
\text { expression }\end{array}$ & $\begin{array}{l}\text { MMP-1, -12, -13 MT1-MMP,67- } \\
\text { kDa laminin receptor }\end{array}$ & (Demeule et al., 2000) \\
\hline $\begin{array}{l}\text { Long chain fatty } \\
\text { acids }\end{array}$ & $\begin{array}{l}\text { catalytic activity } \\
\text { (exosite inhibition) }\end{array}$ & neutrophil elastase, plasmin & (Berton et al., 2001) \\
\hline $\begin{array}{l}\text { Prinomastat AG3340, } \\
\text { (non-peptidomimetic } \\
\text { hydroxamate) }\end{array}$ & catalytic activity & MT1-MMP, MMP-13 & (Shalinsky et al., 1999) \\
\hline $\begin{array}{l}\text { CT1166 } \\
\text { (peptidomimetic) }\end{array}$ & catalytic activity & MMP-3 & (Hill et al., 1995) \\
\hline $\begin{array}{l}\text { Ro 28-2653 } \\
\text { (Pyrimidine-2,4,6- } \\
\text { Trione) }\end{array}$ & catalytic activity & MT1-MMP, MMP-8 & (Grams et al., 2001) \\
\hline $\begin{array}{l}\text { Chemically modified } \\
\text { tetracyclines }\end{array}$ & $\begin{array}{l}\text { catalytic activity, } \\
\text { inhibition of } \\
\text { oxidative activation, } \\
\text { reduction of } \\
\text { expression }\end{array}$ & MT1-MMP & (Sorsa et al., 1998) \\
\hline $\begin{array}{l}\mathrm{N} \text {-sulfonylamino acid } \\
\text { derivatives }\end{array}$ & catalytic activity & MT1-MMP & (Tamura et al., 1998) \\
\hline Bisphosphonates & $\begin{array}{l}\text { catalytic activity, } \\
\text { reduction of } \\
\text { expression }\end{array}$ & $\begin{array}{l}\text { MMP-1, }-3,-7,-8,-12,-13 \text {, and } \\
\text { MT1-MMP }\end{array}$ & (Teronen et al., 1999) \\
\hline Dithiol inhibitors & catalytic activity & MT1-MMP & (Bernardo et al., 2002) \\
\hline $\begin{array}{l}\text { Cysteine switch } \\
\text { peptide }\end{array}$ & catalytic activity & MMPs & (Fotouhi et al., 1994) \\
\hline $\begin{array}{l}\text { CTTHWGFTLC } \\
\text { peptide }\end{array}$ & catalytic activity & & (Koivunen et al., 1999) \\
\hline TSRI265 & $\begin{array}{l}\text { docking of MMP-2 } \\
\text { to } \alpha_{v} \beta_{3} \text { integrin }\end{array}$ & & (Silletti et al., 2001) \\
\hline
\end{tabular}


MMP inhibiting proteins containing domains with structural similarity to TIMPs have been identified. The C-terminal fragment of the progollagen $\mathrm{C}$-terminal proteinase enhancer protein was purified from human brain tumor cells due to its MMP-inhibitory activity, but it is a less potent inhibitor than the TIMPs (Mott et al., 2000). The noncollagenous NC1 domains of collagen type IV are another protein domains with structural similarities to TIMPs (Netzer et al., 1998). Among the NC1 domains of collagen type IV, the $\alpha 3$ chain $\mathrm{NC} 1$ domain is the most potent inhibitor of angiogenesis and tumor growth (Petitclerc et al., 2000). However, the domain also contains RGD-dependent and RGD-independent recognition sites for $\alpha_{V} \beta_{3}$ and $\alpha_{V} \beta_{5}$ integrins and can regulate angiogenesis through integrinmediated signalling (Pedchenko et al., 2003). Tissue factor pathway inhibitor-2, despite being a serine protease inhibitor, can also inhibit MMPs, including the gelatinases (Herman et al., 2001).

Endostatin is a collagen XVIII derived 20-kDa proteolytic fragment with anti-angiogenic and anti-tumor properties (O'Reilly et al., 1997). The protease responsible for the generation of endostatin in vivo is likely cathepsin L (Felbor et al., 2000), but also cathepsin B and MMPs, including MMP-3, MMP-9, MMP-12, MMP-13 and MMP-20 release endostatin in vitro (Ferreras et al., 2000). Endostatin acts as an inhibitor of MMP-2 activation (Kim et al., 2000) as well that of MMP-9 and MMP-13 (Nyberg et al., 2003). It also inhibits the catalytic activity of MMP-2 and MT1-MMP (Kim et al., 2000).

The RECK protein (reversion-inducing cysteine-rich protein with Kazal motifs) is another inhibitor of MMPs, and is the only known membrane-bound MMP inhibitor. RECK is a $110-\mathrm{kDa}$ glycoprotein expressed in many normal tissues, but is absent from transformed and tumor-derived cells (Takahashi et al., 1998). RECK-transfected HT1080 fibrosarcoma cells accumulated only low levels of proMMP-9 in the culture medium and purified RECK bound MMP-9 specifically and inhibited the enzymatic activity of MMP-9 (Takahashi et al., 1998). RECK is also a negative regulator of MMP-2 and MT1-MMP in vivo decreasing angiogenesis and tumor growth. Interestingly, in contrast to MMP deficient animals, deletion of a functional RECK gene is lethal (Oh et al., 2001).

Thrombospondin-1 (TSP-1) is an extracellular 450-kDa glycoprotein with anti-angiogenic properties (Qian et al., 1997). TSP-1 directly binds MMP-9 and inhibits its activation both in vitro and in vivo (Rodriguez-Manzaneque et al., 2001). Consequently, TSP-1-deficient mice show increased angiogenesis and tumor growth, which can be linked to an increased association of vascular endothelial growth factor with VEGFR-2 and appearance of active MMP-9 (Rodriguez-Manzaneque et al., 2001). Contrasting activities have also been reported, as TSP-1 upregulates MMP-9 expression and stimulates invasion of endothelial cells in vitro (Qian et al., 1997). Yeast two-hybrid assays revealed that the thrombospondin type 1 repeats in TSP-1 and TSP-2 interact with the collagen-binding domain of MMP-2 and MMP-9 indicating the potential inhibition mechanism (Bein and Simons, 2000).

A few naturally occuring small-molecule inhibitors of gelatinases have been identified. Neovastat is a shark cartilage extract with anti-angiogenic activity through inhibition of MMPs, although the exact nature of the active ingredient in the extract has not been reported (Dupont et al., 1998). Neovastat has multiple modes of action as it additionally inhibits many VEGF-dependent events in vivo (Falardeau et al., 2001). Matlystatins are produced by an actinomycete strain Actinomadura atramentaria and inhibit gelatinases with an $\mathrm{IC}_{50}$ value less than $1 \mu \mathrm{M}$ (Tanzawa et al., 1992). Aspirin (acetylsalicylic acid) reduces MMP-9 expression and causes inhibition of Epstein-Barr virus latent membrane protein-1 
induced invasiveness of tumor cells in vivo (Murono et al., 2000). Aspirin also suppresses MMP-2 production and reduces in vitro invasiveness of tumor cells (Jiang et al., 2001). However, aspirin does not appear to directly inhibit gelatinases. A potential mechanism for the inhibition is the induction of the RECK protein (Liu et al., 2002). A polyphenolic compound in green tea, epigallocatechin-3-gallate, is a potent inhibitor of gelatinases, but it is not gelatinase selective as it inhibits also other MMPs (Demeule et al., 2000).

Long-chain fatty acids with 10 to 18 carbon atoms inhibit both gelatinases, but only weakly other MMPs as their binding site is in the collagen-binding domain (Berton et al., 2001). In general, the long and unsaturated fatty acids appear to be more potent than the short saturated ones (Berton et al., 2001). However, the long-chain fatty acids are not gelatinase selective gelatinase, as they also inhibit other proteinases including neutrophil elastase and plasmin (Ashe and Zimmerman, 1977; Higazi et al., 1994).

\section{Synthetic gelatinase inhibitors}

Most of the synthetic MMP inhibitors target the catalytic site of the MMPs and act by chelating the catalytically essential zinc ion. Due to the huge interest in the therapeutic intervention of MMPs in cancer, over a hundred small molecule MMP inhibitors have been designed and synthesized (Whittaker et al., 1999). The zinc binding groups that have been utilized in MMP inhibitors include carboxylates, aminocarboxylates, sulfhydryls, thiols, phosphoric acid derivatives and hydroxamates (Whittaker et al., 1999). From these, the hydroxamate-based inhibitors are the most widely used. Batimastat (BB-94) was the first synthetic MMP inhibitor and showed potent antitumor activity in mice (Davies et al., 1993). It is a non-orally bioavailable peptidomimetic hydroxamate inhibitor based on the MMP cleavage site in collagens (Whittaker et al., 1999). The first inhibitors were followed by orally bioavailable inhibitors, such as marimastat. Many non-peptidomimetic MMP inhibitors have also been developed and tested in clinical trials, these include the compounds BAY12-9566, AG3340 and BMS-275291 (Whittaker et al., 1999). Prinomastat (AG3340) is a rather selective gelatinase inhibitor, inhibiting MMP-1, -7 and -11 much less efficiently. However, it shows picomolar affinity to MT1-MMP and MMP-13 (Shalinsky et al., 1999). Other selective active-site inhibitors of gelatinases have also been synthesized (Tamura et al., 1998). These N-sulfonylamino acid derivatives are orally bioavailable and effectively suppress tumor growth in a mouse model, but their inhibitory profile towards other MMPs has not been completely elucidated (Tamura et al., 1998). Two related active site inhibitors with a dithiol structure have been identified as selective gelatinase inhibitors (Bernardo et al., 2002; Rosenblum et al., 2003). Due to the dithiol moiety in these chemicals, they induce a conformational change in the gelatinases, which is not easily reversible (Bernardo et al., 2002).

It is highly difficult to synthesize specific active site inhibitors for an individual MMP. This is because the catalytic sites of MMPs show remarkable similarity, which is also reflected by the overlapping substrate specificity of the MMPs. The X-ray structures of several MMPs have established that the S1' subsite in the catalytic site is the main determinant of the substrate specificity as well as a selectivity determinant for the inhibitors. Based on the S1' subsites, MMPs can be divided into deep pocket and shallow pocket containing inhibitors. MMP-2, -3, -8, -9, -13 and MT1-MMP contain a deep pocket, whereas MMP-1 and MMP-7 have a shallow S1' pocket (Zucker et al., 2000). Other investigators further divide the MMPs into intermediate pocket containing enzymes, where MMP-2, -8, -9 and - 
26 are classified as intermediate ones (Park et al., 2003). Differences in other sites such as the S2 site can be further utilized in order to increase the selectivity of the inhibitors (Chen et al., 2003; Kridel et al., 2001).

Tetracyclines, which in addition to their antimicrobial activity inhibit inflammatory cell migration and chemotaxis to sites of inflammation, act also as MMP inhibitors. The ability of tetracyclines to inhibit MMPs is independent of their anti-microbial activity (Sorsa et al., 1998). The tetracyclines act on two levels, they suppress the gelatinase expression (Seftor et al., 1998) and directly inhibit gelatinase activity trough a zinc-chelating effect (Sorsa et al., 1998). The tetracycline derivatives have entered clinical trials as MMP-inhibitors (Cianfrocca et al., 2002). Clodronate and other bisphosphonates have been developed to treat bone diseases due to their ability to inhibit bone resorption. However, it has been also found that these compounds directly inhibit MMP activity (Teronen et al., 1999).

The cysteine switch peptide MRKPRCGVPDVG from the prodomain of MMP-3 was the first peptide used to block the enzymatic activity of the MMPs (Fotouhi et al., 1994), whereas the phage display-derived CTTHWGFTLC (CTT) peptide was the first gelatinaseselective peptide inhibitor (Koivunen et al., 1999). The CTT peptide did not inhibit the activity of MT1-MMP, MMP-8 or MMP-13. The mechanism how CTT inhibits gelatinase activity is not known. The CTT peptide was enriched in a biopanning with active MMP-9 and was the most potent inhibitor among the peptides containing a WGF motif. The CTT peptide inhibited the migration of several cell lines in vitro and retarded tumor progression in mouse models. It also exhibited a strong tumor homing ability in comparison to the normal tissues (Koivunen et al., 1999). The targeting capability of CTT was further demonstrated with liposomes coated with the CTT peptide. These liposomes efficiently targeted gelatinase-expressing cancer cells in vitro (Medina et al., 2001). The CTT peptide has also been used to modify the natural tropism of adenovirus for a therapeutic gene delivery in a rabbit restenosis model (Turunen et al., 2002). In addition, CTT peptide has been used to localize gelatinase activity in tissue samples using in situ zymography (Pirilä et al., 2001), and to evaluate the contribution of gelatinases in various biological processes including vasoconstriction, epithelial-mesenchymal transition and hepatitis (Cheng and Lovett, 2003; Fernandez-Patron et al., 2000; Franzke et al., 2002).

There are several RNA-based strategies to inhibit the gelatinases. Ribozymes, RNA molecules with catalytic activity, have been utilized to inhibit translation of the gelatinases. Importantly, the MMP-9 down-regulated cells retained their tumorigenicity but were no longer able to metastasize (Hua and Muschel, 1996; Sehgal et al., 1998). MMP-2 has also been targeted with a ribozyme approach. MMP-2, but not MMP-9 was found to be necessary for glomerular mesangial cell proliferation and differentiation (Turck et al., 1996). Similarly, adenoviral delivery of antisense mRNA of MMP-9 effectively suppressed tumor xenograft growth in vivo (Lakka et al., 2002). Small interfering RNAs have also been used to specifically silence MMP-9. Lack of MMP-9 caused a decrease in spreading of Ewings sarcoma cells, inhibition of chemotactic migration towards fibronectin and induction of E-cadherin mediated cell aggregation (Sanceau et al., 2003). Therapeutic inhibition of MMPs may also be achieved by other indirect means. These include targeting of extracellular factors, signal-transduction pathways or nuclear factors that are required for the transcriptional activation of MMPs. Another possibility is to inhibit the activity of the MMP activating proteases (reviewed by Overall and Lopez-Otin, 2002). However, those strategies aiming at suppressing MMP transcription may have to take into account that the stromal cells are often the producers of the MMPs in the tumor. 


\section{CELL MIGRATION AND INVASION}

\section{Regulation of cell migration}

Cell migration is a complex process involving many types of components both intra- and extracellularly and the signalling events linking these. More specifically, cancer cell migration can be viewed as a process regulated by matrix-degrading proteinases, integrins and other cell adhesion molecules (Chapman, 1997). As migration is a critical event in cancer progression and especially metastasis, inhibition of cell migration represents an attractive therapeutic target. The basic concepts in cell migration are well established, but the details how these processes are regulated and executed are far from clear. The main principle of cell migration is simple; the cell must convert the intracellular forces generated by the rearrangement of the actin cytoskeleton to cell body translocation (Lauffenburger and Horwitz, 1996). The cells typically migrate in response to migratory signals. This cellular response can be either non-directional movement (chemokinesis) or directed migration along a chemical concentration gradient of the signal inducer (chemotaxis). In the initial phase of cell migration, the cells polarize and extend membrane processes such as lamellipodia and filopodia at the cell front. Lamellipodia are broad, sheet-like structures whereas filopodia are thin cylindrical needle-like projections (Lauffenburger and Horwitz, 1996). Invadopodia are a specialized form of small needle-like projections in invasive cells being located beneath the cells rather than in the cell edge in a two-dimensional culture (Chen and Wang, 1999). The invadopodia have been characterized as highly dynamic structures where proteolytic degradation takes place (Chen, 1996; Chen and Wang, 1999; Mueller et al., 1999). Localized matrix degradation takes place also in the leading edge of the membrane extensions together with concomitant formation of nascent adhesive contacts by integrins and other adhesion molecules (Lauffenburger and Horwitz, 1996; Regen and Horwitz, 1992). These nascent adhesive sites may further develop to mature focal contacts, which are a highly dynamic protein network containing over fifty different proteins (Zamir and Geiger, 2001).

Integrins are one of the key players in the regulation of cell migration. Integrins are a large family of heterodimeric cell adhesion molecules composed of an $\alpha$ chain and a $\beta$ chain (Figure 5). Various combinations of the $\alpha$ and $\beta$ chains bind specific cell surface and ECM ligands and transmit signals between the outside and inside of the cells (Giancotti and Ruoslahti, 1999; Hynes, 2002). For example, $\alpha_{5} \beta_{1}$ binds fibronectin, whereas the $\alpha_{V} \beta_{5}$ is a vitronectin receptor. The ligand binding to integrins typically occurs through negatively charged residues present in the ligand such as the RGD motif (Arnaout et al., 2002; Hynes, 2002). The binding site of these ligands is either the I domain in the $\alpha$ subunit of integrins or a binding pocket formed by the $\alpha$ and $\beta$ subunit together as visualized in the $\alpha_{v} \beta_{3}$ integrin structure with the RGD peptide (Xiong et al., 2002). I domain-containing integrin $\alpha$ subunits include $\alpha_{1}, \alpha_{2}, \alpha_{10}, \alpha_{11}, \alpha_{\mathrm{L}}, \alpha_{\mathrm{X}}, \alpha_{\mathrm{D}}, \alpha_{\mathrm{M}}$ and $\alpha_{\mathrm{E}}$. All the other integrin $\alpha$ subunits lack an I domain (Hynes, 2002). Many integrins recognize a three amino acid motif RGD present in certain matrix proteins (Ruoslahti, 1996). RGD containing peptides have also been found by biopanning with these integrins (Healy et al., 1995; Koivunen et al., 1993; Koivunen et al., 1995), whereas leukocyte $\beta_{2}$ integrins recognize an LLG motif found by phage display (Koivunen et al., 2001). Studies with the leukocyte-specific $\beta_{2}$ integrins have provided important information about integrin structure and function (Gahmberg, 1997). These integrins are also clinically highly relevant. Consequently, antagonists of the leukocyte 
integrins $\alpha_{\mathrm{L}} \beta_{2}$ and $\alpha_{\mathrm{M}} \beta_{2}$ are being developed for the treatment of various autoimmune diseases and inflammatory conditions (Bansal et al., 2003; Shimaoka and Springer, 2003).

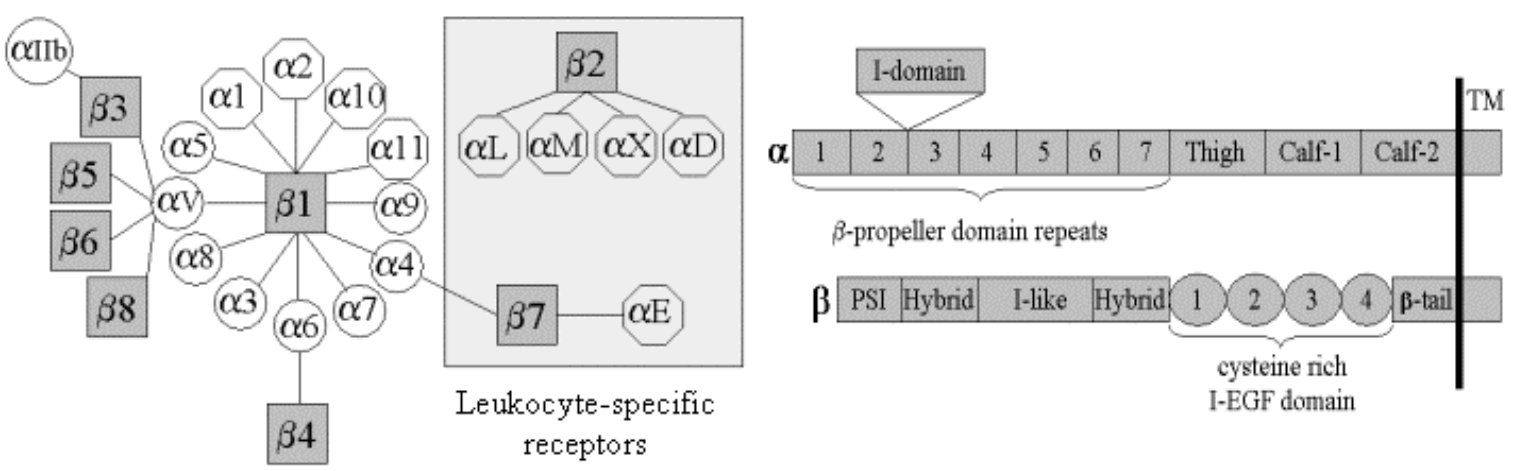

Figure 5. Different combinations of integrin $\alpha$ and $\beta$ subunits, and the domain organisation of the subunits. The I domain containing $\alpha$ subunits are shown with octagons and the leukocytespecific integrins are shaded with the grey box. PSI, plexin/semaphorin/integrin -homology domain; TM, transmembrane domain. Figure modified from (Hynes 2002, Shimaoka and Springer, 2003).

The formation of the adhesive contacts is regulated by members of the Rho subfamily of GTPases, including Cdc42, Rac and Rho (Hall, 1994). For example, the Rho protein directly controls the formation of focal adhesions and actin stress fibers (Ridley and Hall, 1992). Not surprisingly, members of the rho family have frequently been associated with tumor metastasis (Clark et al., 2000; Jaffe and Hall, 2002; Suyama et al., 2003). Formation of the adhesive structures is accompanied by tyrosine phosphorylation of cytoskeleton-associated proteins such as focal adhesion kinase (FAK), paxillin and tensin, which are important mediators of intracellular signalling (Weisberg et al., 1997). FAK is a $125-\mathrm{kDa}$ non-receptor tyrosine kinase that can be activated through integrin-mediated signals and regulates multiple functions such as cell motility, survival and proliferation (Giancotti and Ruoslahti, 1999; Parsons et al., 2000). Increased FAK expression is a potent marker for the invasiveness of human tumors (Owens et al., 1995) and inhibition of FAK signalling through overexpression of a dominant negative mutant causes tumor dormancy (Aguirre Ghiso et al., 1999). FAK enhances cell motility and invasion by distinct mechanisms. FAK negative fibroblasts are defective in migration, but expression of viral Src-protein restores the motility through a reactivation of signalling through Src. The Src kinase associates with uPAR and integrins (Wei et al., 1999). Src acts by linking FAK to integrins, such as $\alpha_{\mathrm{V}} \beta_{5}$ in VEGF mediated signalling (Eliceiri et al., 2002). However, invasion of FAK negative fibroblasts and expression of the gelatinases is not restored by v-Src. To become invasive, fibroblast cells require transient accumulation of FAK in lamellipodia and formation of FAK-Src-p130Cas-Dock180 signalling complex together with Rac activation (Hsia et al., 2003). The authors suggested that FAK activity is required for the synchronization of cell motility and invasive behaviour. Recently, MT1-MMP and MT3-MMP activity was linked to proteolytic cleavage of FAK in vascular smooth muscle cells. However, it was not established if the cleavage was directly caused by the MT-MMPs (Shofuda et al., 2004).

Generation of new adhesive sites is necessary but not sufficient for cell migration. The cells also need a mechanism to release the adhesions in the rear of the cells. In migrating fibroblasts, a major part of the integrins is left on the substratum by a mechanism called "membrane ripping" (Chen, 1981; Lauffenburger and Horwitz, 1996). The rest of the integrins are released from the substratum and re-distributed on the cell surface or 
endocytosed (Palecek et al., 1996). The mechanism of the rear release potentially involves multiple mechanisms, including mechanical stress from the cytoskeleton and signalling pathways regulating integrin affinity (Lauffenburger and Horwitz, 1996). Proteases and protease inhibitors may contribute to this process. For example, the plasminogen activator inhibitor-1 can directly cause cellular detachment by inactivating the integrins (Czekay et al., 2003). Only a few other proteins have been shown to directly participate in cell detachment, namely tenascin-C, thrombospondin-1 and -2 and SPARC (secreted protein, acidic and rich in cysteine) (Murphy-Ullrich, 2001). The proteases can also indirectly modulate the affinity and hence the detachment of the cells by processing the extracellular matrix (Giannelli et al., 1997) or by cleaving integrin associated molecules (Andolfo et al., 2002; Montuori et al., 2002).

Thus, adhesion and detachment controlled by integrin-ligand interactions are one of the key regulators of cell migration. Migration of cells and the speed of migration can be regulated by ligand levels, integrin levels and the integrin-ligand affinities. Experimentally, the migration speed is biphasic, too little or too much adhesion strength will decrease the cell velocity, irrespective if this has been obtained by increasing ligand or integrin concentration or the integrin affinity to the ligand (Palecek et al., 1997). Indeed, these studies suggest that relatively small changes in integrin expression or affinity can substantially alter the speed of migration. Furthermore, inhibition of cell migration can thus be obtained not only with integrin-function blocking antibodies but also with antibodies that induce the activation of integrins (Palecek et al., 1997) and proteases that change the affinity of the matrix ligand to the integrin (Schenk and Quaranta, 2003).

A possible complication in cancer therapy with cell migration inhibiting agents is that the migration mechanisms utilized by the cancerous cells and non-neoplastic cell are highly similar or identical. Migration of non-neoplastic cells is required for example in embryogenesis, inflammation and wound healing. Hence, inhibition of these activities may have detrimental side-effects (Friedl and Brocker, 2000; Lauffenburger and Horwitz, 1996).

\section{Multiple roles of proteinases in cell migration and invasion}

Tumor invasion is defined as penetration of the tissue barriers, such as the basement membrane by the migrating cancerous cells (Dano et al., 1985; Mignatti and Rifkin, 1993; Wolf et al., 2003). As discussed above, cell migration and invasion are distinct but coordinately regulated phenomena (Hsia et al., 2003). During the tumor progression, invasive capacity is required at multiple steps. Tumor cells frequently invade the surrounding tissue when the tumor starts to grow. Next, the capillary endothelial cells must invade the tumor and create the tumor blood vessels. Thereafter, some tumor cells intravasate into the blood circulation for metastasis, whereas the host immune cells invade the tumor. Last, the tumor cells must arrest in the distant organs, extravasate and migrate into the new metastatic site and start the invasive cycle again (Mignatti and Rifkin, 1993). Typically, alternating cycles of proteolysis and its inhibition occur in the tissues in order to control the protease activity. It was originally thought that the protease activity is only required for the degradation of the underlying matrix. It has now become evident that proteases also generate promigratory signals by cleavage of latent growth factors or by disrupting cell-cell contacts mediated by E-cadherin (Figure 6). The gelatinases actively participate in the activation of latent growth factors, MMP-9 being able to release active VEGF and TGF- $\beta$, thus promoting angiogenesis and tumor growth (Bergers et al., 2000; Yu 
and Stamenkovic, 2000). The proteases can also release protein fragments and growth factors with chemotactic activity from the ECM, and expose migration promoting cryptic epitopes (Schenk and Quaranta, 2003; Stetler-Stevenson and Yu, 2001).

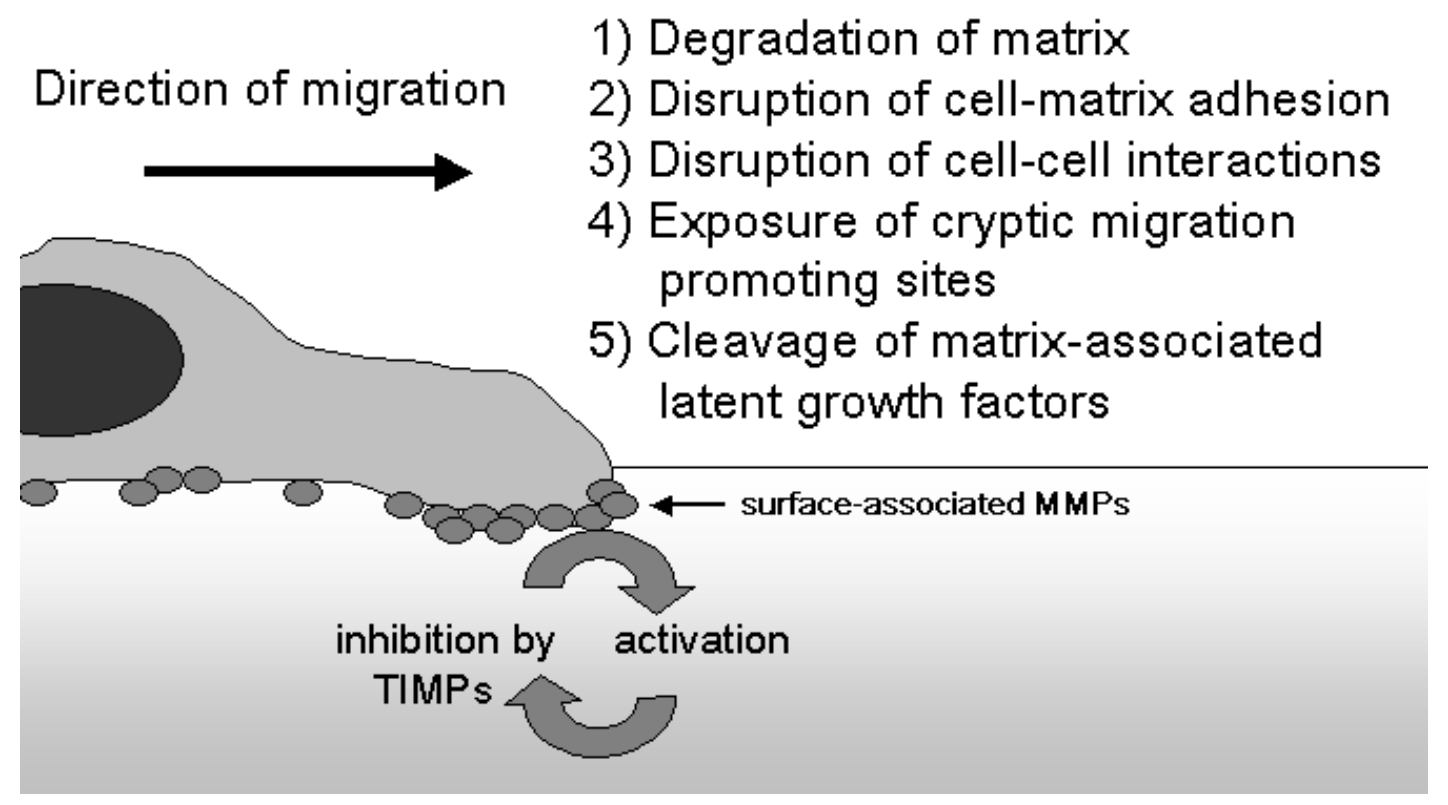

Figure 6. Functions of MMPs in cell migration and invasion.

Although proteases clearly stimulate cell migration in several ocassions, current evidence suggests that protease activity per se is not always essential. It has become clear that protease-independent migration strategies exist. Recently it was shown that catalytically inactive MT1-MMP mutant supported cell migration similarly to the wild type enzyme and the cell migration supporting activity was accounted for the catalytic domain and the Cterminal domain (Cao et al., 2004). Other investigators have similarly suggested a migration inducing ability of MMP-9 independent of the catalytic activity (Sanceau et al., 2003). Despite complete pharmacological inhibition of protease activity, many cells continue to migrate by utilizing existing pathways in the matrix and migrating by an ameboid-like movement. For example, T cells, HT1080 fibrosarcoma cells and MDA-MB-231 breast carcinoma cells can migrate in this manner (Wolf et al., 2003). Whether proteaseinteractions that are independent of the proteolytic activity are required in this kind of migration is not known. The protease-independent mode of cell migration may explain the observations that MMP-9 is required for neutrophil transmigration through the endothelium in some but not all in vivo models (Betsuyaku et al., 1999; D'Haese et al., 2000).

The gelatinases are also linked to the cell spreading and cytoskeletal changes during cell migration. Activated RhoA, the regulator of focal adhesions is necessary, but not sufficient for invasion (Stam et al., 1998). MMP-9 colocalizes with RhoA, which is a regulator of cell spreading in endothelial cells and expression of a constitutively active RhoA increases MMP-9 secretion (Abecassis et al., 2003). However, these results are contradictory to the results with RNA interference of MMP-9, which show that RhoA is inactive in the presence of MMP-9 and inhibition of MMP-9 expression decreases cell spreading (Sanceau et al., 2003). It has been shown that inhibition of Rho by overexpression of a dominant negative mutant inhibits invasion but as well overexpression of Rho reduces invasiveness (Banyard et al., 2000). These contradictory results may be explained by the finding that fluctuating levels of active Rho, rather than constitutively active Rho, are required for efficient invasion 
(Lin et al., 1999). Other evidence for the involvement of gelatinases in cell spreading comes from the studies with MMP inhibitors. MMP-2 inhibition by overexpression of TIMP-2 causes extensive spreading of cells (Ray and Stetler-Stevenson, 1995). Many proteases, including the gelatinases accumulate into focal adhesions. For example, the gelatinases are found in the focal adhesions of endothelial cells (Partridge et al., 1997), and both TIMPs and chemical MMP-inhibitors stabilize the focal adhesion contacts of fibroblasts (Ho et al., 2001). Conversely, overexpression of MMPs may destabilize focal adhesions (Shofuda et al., 2004). It has been suggested that MMP inhibitors augment cell adhesion by preventing cadherin cleavage and stabilize cell-cell contacts by inhibiting ECM degradation and thereby maintain integrin-ECM adhesion and focal contact assembly (Ho et al., 2001).

\section{Cell surface associations of the gelatinases}

Controlling of the proteolytic activity at the cell surface greatly facilitates cell migration and invasion (Werb, 1997). Docking of the proteases on the cell surface provides a direct mechanism by which cells can utilize and direct the proteolytic activity into correct substrates. The cell surface binding may additionally protect the proteases from the action of soluble inhibitors by steric hindrance, although some inhibitors like TIMP-2 have relatively free access to the cell surface. It has been also shown that proteases may be released from the cells in such a high concentration that the extracellular inhibitor concentration is locally exceeded. Consequently a portion of the proteases remains uninhibited and is capable of focalized pericellular proteolysis for a short duration. This phenomenon has been called "quantum proteolysis" (Liou and Campbell, 1996).

As mentioned earlier, stimulation of tumor cells with phorbol esters or growth factors induces proMMP-9 secretion. A small part of the secreted proMMP-9 is consequently observed on the cell surface of endothelial cells (Olson et al., 1998; Partridge et al., 1997), keratinocytes (Mäkelä et al., 1998), breast epithelial (Olson et al., 1998; Toth et al., 1997) and breast cancer cells (Mira et al., 1999), neutrophils (Gaudin et al., 1997; Owen et al., 2003), and many types of cancer cells including pancreatic (Zucker et al., 1990), ovarian (Ellerbroek et al., 2001) and prostate cancer (Festuccia et al., 2000), mammary carcinoma (Yu and Stamenkovic, 1999; Yu and Stamenkovic, 2000), promyelotic leukemia (Fiore et al., 2002) and fibrosarcoma cells (Mazzieri et al., 1997). The cell surface-bound gelatinases play a role in cell migration. For example, human bronchial epithelial cells secrete MMP-9 in an actin-dependent manner to the leading edge of migrating cells. MMP-9 activity in these cells was required specifically for cell migration and not adhesion or spreading (Legrand et al., 1999). Although the mechanisms by which the MMP-9 is localized on the cell surface appear to be redundant, there are a few important similarities. First, it appears that the cell-surface bound MMP-9 is often free of TIMP-1. This has been observed in breast epithelial cells as well as in neutrophils (Owen et al., 2003; Toth et al., 1997). Second, in most studies cell surface localized MMP-9 is found in the proenzyme form (Gaudin et al., 1997; Mazzieri et al., 1997; Olson et al., 1998; Toth et al., 1997; Zucker et al., 1990). The inhibitor-free proenzyme is thought to be highly susceptible for activation.

Multiple binding mechanisms of MMP-2 and -9 on the cell surface have been identified (Figure 7) On the surface of MCF10A breast epithelial cells, HT1080 fibrosarcoma and other tumor cells, proMMP-9 can associate with the $\alpha 2(\mathrm{IV})$ chain of collagen type IV, whereas the affinity of MMP-2 to this collagen chain is much lower (Olson et al., 1998; Toth et al., 1999). This interaction is likely mediated through the collagen-binding domain 
as TIMP-1 does not inhibit this interaction (Olson et al., 1998). The CBD of MMP-2 is also utilized for binding to the cell surface of normal fibroblasts. In a coculture system, fibronectin present on the surface of cancer cells competes with the fibroblast-associated MMP-2 liberating soluble MMP-2 (Saad et al., 2002). On the fibroblast surface, MMP-2 binds to collagens, likely the $\alpha 1$ and $\alpha 2$ chains of type I collagen, with a possible involvement of $\beta_{1}$ integrins binding to these collagen chains (Steffensen et al., 1998). Again, the CBD-mediated cell surface association of MMP-2 appears to be TIMPindependent, as the activation of MMP-2 on fibroblasts is markedly elevated by competing the cell surface bound MMP-2 with recombinant CBD (Steffensen et al., 1998).

A specific splicing variant of the hyaluronan receptor CD44 is involved in cell-surface association of MMP-9 in mouse mammary carcinoma and human melanoma cells. Disruption of this binding by overexpression of a soluble CD44 inhibits tumor invasion in vivo ( $\mathrm{Yu}$ and Stamenkovic, 1999). MT1-MMP may regulate this interaction as it proteolytically processes CD44. Curiously, this cleavage results in enhanced cell migration (Kajita et al., 2001). The interactions of CD44 with MMP-9 are complex, as it has been observed that in osteoclast-like cells hyaluronan binding to CD44 downregulates MMP-9 expression (Spessotto et al., 2002). The interaction mechanism of CD44 with MMP-9 is not known, however, MT1-MMP utilizes the hemopexin-like domain for its interaction with CD44 (Mori et al., 2002). MMP-9 interacts with CD44 in invadopodia (Bourguignon et al., 1998). These are the same cellular structures, which also contain MMP-2 and $\alpha_{\mathrm{V}} \beta_{3}$ complexes (Deryugina et al., 2001) together with MT1-MMP (Nakahara et al., 1997). In leukemic cells, proMMP-9 has been observed to interact with intercellular adhesion molecule-1 (ICAM-1), which was identified as a substrate for MMP-9 (Fiore et al., 2002). Shedding of ICAM-1 by MMP-9 was found to enhance tumor cell resistance to natural killer cell-mediated cytotoxicity indicating an additional mechanism whereby MMP-9 may affect tumor growth (Fiore et al., 2002).

Another cell surface receptor for the gelatinases is the low-density lipoprotein-related scavenger receptor (LRP). As mentioned before, MMP-2 and -9 can be internalized through this receptor (Hahn-Dantona et al., 2001; Yang et al., 2001). Although in most cases the gelatinases promote cell migration and invasion, other activities of the gelatinases may counteract this effect. For example, MMP-9 has been found to inhibit corneal reepithelialization by controlling cell replication (Mohan et al., 2002). Thus, it is not clear how the endocytic removal of gelatinases affects cell migration and invasion. The RECK protein is another cell-surface receptor for MMP-9 and MMP-2 although direct experimental evidence demonstrating the interactions of gelatinases with RECK is still lacking. Because RECK inhibits MMP activity, it probably preferentially binds the active gelatinases. Other receptors for gelatinases may also exist. MT2-MMP mediated activation of MMP-2 involves TIMP-independent C-terminal domain interactions on the cell surface, but the receptor has not been identified (Morrison et al., 2001).

Integrins not only recognize various structural proteins, but they also act as receptors for proteases, including the MMPs. The $\alpha_{\mathrm{V}} \beta_{3}$ integrin recognizes the C-terminal domain of MMP-2 and is able to localize MMP-2 on the cell surface. More specifically, MMP-2 localizes with MT1-MMP, TIMP-2 and $\alpha_{\mathrm{V}} \beta_{3}$ integrin in specific membrane microdomains called caveolae as well as in invadopodia and in the leading edge of the migrating cells (Nabeshima et al., 2000; Puyraimond et al., 2001). In some models, the cell surface activity of MMP-2 was found to be dependent on the $\alpha_{\mathrm{V}} \beta_{3}$ integrin interaction and this interaction was necessary for tumor angiogenesis (Brooks et al., 1998; Brooks et al., 1996). Delivery of 
the MMP-2 C-terminal domain as a recombinant protein or via viral infection also potently suppressed angiogenesis (Pfeifer et al., 2000). The C-terminal domain of MMP-2 appears to be a naturally occuring proteolytic fragment and an inhibitor of pericellular MMP-2 activity (Bello et al., 2001; Brooks et al., 1998). A small molecule inhibitor named TSRI265 has been identified as a compound being able to block the interaction of MMP-2 and $\alpha_{V} \beta_{3}$ integrin, but it did not inhibit MMP-2 activity. A labelled derivative of TSRI265 bound to the $\alpha_{V} \beta_{3}$ integrin and not to MMP-2. Similarly to the C-terminal domain of MMP-2, TSRI265 inhibited angiogenesis indicating that MMP-2 must be localized on the cell surface to perform at least some of its biological functions (Boger et al., 2001; Silletti et al., 2001).

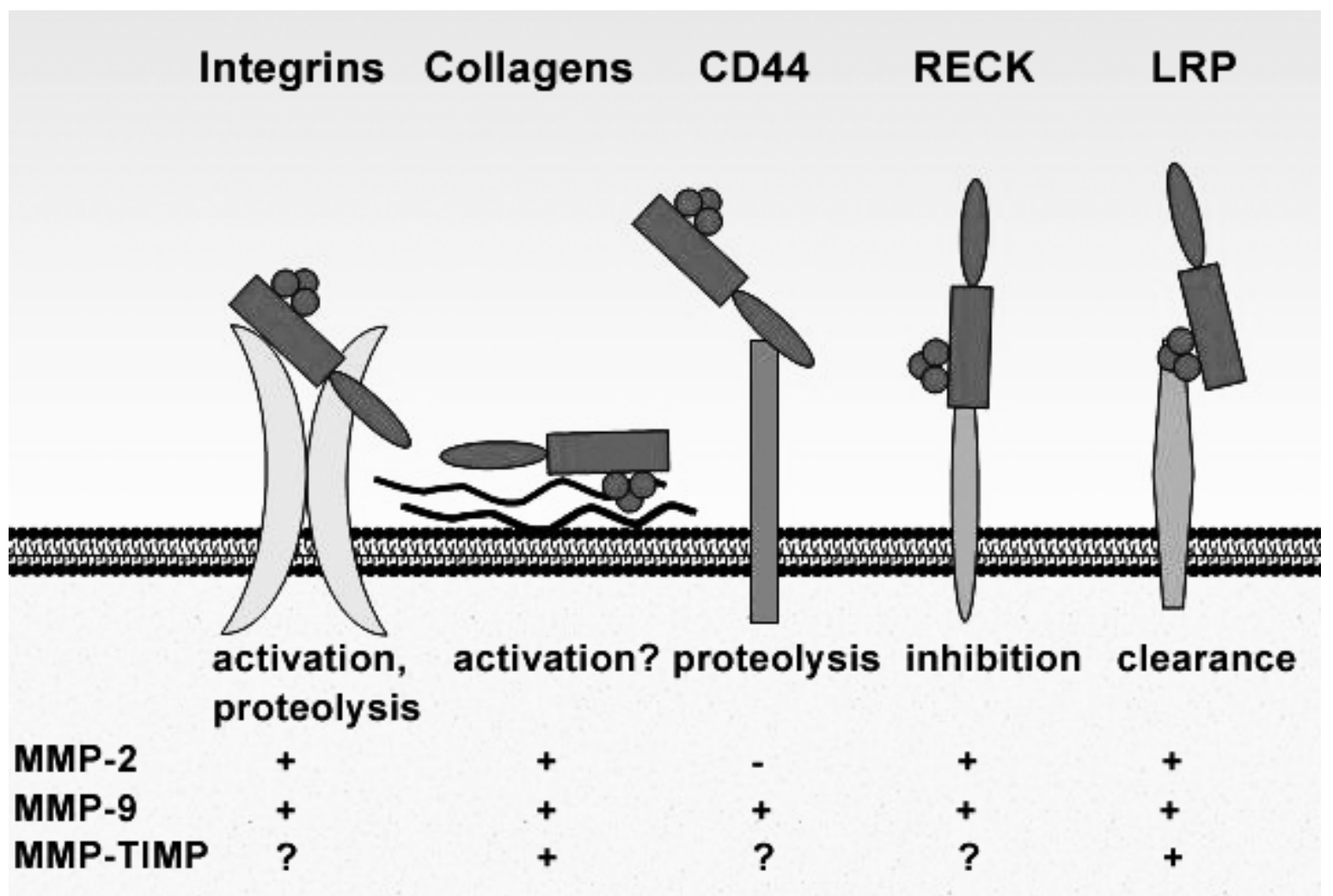

Figure 7. Cell surface interactions of the gelatinases. Many of the functions and interaction mechanisms are still hypothetical. Additional binding partners such as MT1-MMP (Zucker et al., 2003) have been omitted for simplicity (see text for details).

Interestingly, the binding of MMP-2 C-terminal domain to the $\alpha_{V} \beta_{3}$ integrin is RGDindependent and does not compete with vitronectin binding to the $\alpha_{v} \beta_{3}$. Neither does the TSRI265 affect vitronectin binding (Brooks et al., 1998; Silletti et al., 2001). RGDindependent binding to $\alpha_{\mathrm{v}} \beta_{3}$ integrin is not unique for MMP- 2 as the binding of tumstatin, a proteolytic fragment of type IV collagen $\alpha 3$ chain is RGD-independent (Maeshima et al., 2001). Unfortunately, the binding sites of MMP-2 and tumstatin in the integrin have not been elucidated. The presence of two or more binding sites in the $\alpha_{V} \beta_{3}$ integrin suggests that integrins are able to gather many proteins to a single complex. This is apparently a necessary trait for efficient cell migration.

MMP-9 has been observed to associate with $\alpha_{5} \beta_{1}$ integrin in epithelial cells grown on fibronectin. The association could be modulated with gangliosides, suggesting that MMP-9 localizes to specific cell surface lipid microdomains (Dolo et al., 2000; Wang et al., 2003). However, direct interactions of MMP-9 with $\alpha_{5} \beta_{1}$ integrin or any other integrins have not 
been demonstrated. Activated $\alpha_{V} \beta_{3}$ functionally co-operates with MMP-9, because MMP-9 stimulates $\alpha_{V} \beta_{3}$-dependent migration of breast cancer cells (Rolli et al., 2003). Similarly, cooperation of $\alpha_{3} \beta_{1}$ integrin and MMP-9 in tumor invasion has been observed (Morini et al., 2000). MMP-9 also cooperates with $\alpha_{v} \beta_{5}$ integrin. Insulin like growth factor-1 (IGF-1) mediated induction of cell migration coincides with MMP-9 expression and activity in MCF-7 breast cancer cells. The migration was specifically inhibited by a broad spectrum MMP inhibitor (Mira et al., 1999). However, MMP-9 is not absolutely required for the IGF1 mediated migration, as CS-1 melanoma cells transfected with $\alpha_{v} \beta_{5}$ integrin also migrate in response to the IGF-1 stimulation (Brooks et al., 1997) despite not expressing MMP-9 and only minimally MMP-2 (Brooks et al., 1998). Of considerable interest is the finding that there are two angiogenic pathways, which are differentially regulated by the $\alpha_{\mathrm{V}} \beta_{3}$ and $\alpha_{\mathrm{V}} \beta_{5}$ integrins (Friedlander et al., 1995). Angiogenesis induced by bFGF and TNF- $\alpha$ is mediated through $\alpha_{V} \beta_{3}$ integrins, whereas VEGF, TGF- $\alpha$ and phorbol ester acting through protein kinase $C$ act through $\alpha_{V} \beta_{5}$ integrins. Given that MMP-2 interaction with $\alpha_{V} \beta_{3}$ integrins is critical for the angiogenic response, it is possible that MMP-9 could be similarly involved in the $\alpha_{V} \beta_{5}$ integrin mediated angiogenic response as MMP-9 expression is induced by protein kinase $\mathrm{C}$ activation.

\section{Other proteases in cell migration and invasion}

Cell surface protease activity is typically observed in highly migratory cells and it is localized in the leading edge of the migrating cells as well as invadopodia (Artym et al., 2002; Mueller et al., 1999). After the initial finding that MMP-2 can directly interact with the $\alpha_{\mathrm{V}} \beta_{3}$ integrin, it has been found that several other proteases function similarly. MMP-1 binds to $\alpha_{1} \beta_{1}$ and $\alpha_{2} \beta_{1}$ integrins (Stricker et al., 2001), whereas MT1-MMP associates with $\beta_{3}$ or $\beta_{1}$ integrins on endothelial cells depending on the extracellular matrix (Galvez et al., 2002). Other proteases than MMPs may also interact with integrins. These include UPA/uPAR, which may interact with several integrins (Aguirre Ghiso et al., 1999; Carriero et al., 1999; Wei et al., 2001; Wei et al., 1996; Xue et al., 1997), elastase with $\alpha_{M} \beta_{2}$ integrin (Cai and Wright, 1996), snake venom disintegrin/metalloproteinase with $\alpha_{2} \beta_{1}$ integrin (Ivaska et al., 1999) and ADAMs with several integrins (Bax et al., 2004; Bridges et al., 2002; Nath et al., 1999).

The serine protease urokinase-plasminogen activator (UPA) together with its receptor UPAR is one of the most characterized proteolytic systems. Initially uPA and UPAR were thought to be mainly responsible for plasminogen activation on the cell surface, but later studies have shown that uPA and UPAR have also direct and essential roles in cell adhesion, migration, differentiation and proliferation. Many of these functions appear to be signallingdependent processes and independent on the proteolytic activity (Blasi and Carmeliet, 2002). uPA is expressed as a single chain precursor, which may be activated by many proteases including plasmin, trypsin or the gelatinases (Dano et al., 1985; Ellis, 2003; Koivunen et al., 1989; Prager et al., 2003). The single chain uPA can be cleaved either to a $52 \mathrm{kDa}$ or $33 \mathrm{kDa}$ active two-chain molecule. uPAR is a GPI-anchored membrane protein and responsible for the localized plasminogen activation on the cell surface. More specifically, uPA and UPAR are localized in focal contacts and at areas of cell-cell contacts (Pöllänen et al., 1988; Pöllänen et al., 1987). The binding of uPA to uPAR greatly accelerates plasminogen activation because the receptor-bound uPA appears to mediate the assembly of catalytically favored complexes with cell-associated plasminogen (Ellis et al., 1999). The ability of uPAR to mediate various signals inside the cells in the absence of an intracellular domain is still incompletely understood. It appears that the signalling is largely 
mediated through integrins, which interact with UPAR on the cell surface. It was initially found that uPAR binds to $\beta_{1}$ and $\beta_{2}$ integrins and regulates integrin functions (Wei et al., 1996). Later studies revealed that $\beta_{3}$ and $\beta_{5}$ integrins also associate with uPAR (Aguirre Ghiso et al., 1999; Carriero et al., 1999; Wei et al., 2001; Xue et al., 1997). Interestingly, the association of UPA/uPAR system with integrins is dependent on the ECM and directly influences the migration of cells (Wei et al., 2001; Xue et al., 1997). Expression levels of uPAR in vivo are directly related to their invasiveness and the rate of the tumor growth. UPAR deficient cells cease to grow and enter a state of dormancy in vivo (Yu et al., 1997) and this effect is mediated by the $\alpha_{5} \beta_{1}$ integrin and MAPK signalling (Aguirre Ghiso et al., 1999). Down-regulation of uPAR also reduces the intravasation of tumor cells in a chicken chorionallantoic membrane (CAM) model (Kim et al., 1998).

uPA and its receptor uPAR are also able to regulate the activation-state of the integrins. The order of events occuring on the cell surface is far from clear. Some studies show that integrin activation by antibodies or divalent cations blocks uPA and MMP-2 activation and that MMP-2 activates uPA (Prager et al., 2003; Yan et al., 2000). This suggests that uPA and MMPs are activated prior to integrin activation and remain associated to the integrins. Furthermore, uPAR can be cleaved by uPA (Hoyer-Hansen et al., 1992) or MMPs in vitro (Andolfo et al., 2002; Koolwijk et al., 2001) and the cleaved uPAR form is also found in invasive tumor xenografts (Solberg et al., 1994). The cleavage occurs between domains 1 and 2 impairs uPA and integrin binding (Montuori et al., 2002) and exposes a chemotactic epitope (Fazioli et al., 1997) suggesting that this cleavage is one of the cell migration regulating events on the cell surface.

A number of other serine proteases have been implicated in tumor invasion. Trypsin-2 is a tumor-associated protease (Koivunen et al., 1989), which activates MMP-1, -3, -8, -9 and 13 (Moilanen et al., 2003; Sorsa et al., 1997) and pro-urokinase (Koivunen et al., 1989). Trypsin activation of MMP-9 enhances tongue carcinoma cell invasion in the CAM model (Nyberg et al., 2002). Seprase is a membrane-bound serine-protease with gelatinase activity (Monsky et al., 1994). It associates with $\alpha_{3} \beta_{1}$ integrins on collagen type I matrix in the invadopodia of invasive melanoma cells (Mueller et al., 1999). In addition, seprase associates with uPAR and dipeptidyl peptidase IV regulating invasion and migration of fibroblast cells (Ghersi et al., 2002). Seprase activity is also associated with increased metastasis in vivo (Iwasa et al., 2003). Hepsin is a type II transmembrane serine protease normally expressed in hepatocytes (Leytus et al., 1988). Microarray analyses have consistently found it to be one of the most upregulated genes in advanced prostate cancer (Dhanasekaran et al., 2001; Magee et al., 2001). Other transmembrane serine proteases implicated in cancer progression are membrane-type serine protease-1/matriptase in breast cancer (Shi et al., 1993), TMPRSS2 in prostate cancer (Afar et al., 2001) and TMPRSS4 in pancreatic cancer (Gress et al., 1997).

Cathepsin B, a lysosomal cysteine protease can be localized on the surface of invasive cells through an interaction with annexin II tetramer (Mai et al., 2000). Cathepsin B can degrade many ECM proteins as well as activate other proteases on the cell surface (Podgorski and Sloane, 2003). ADAMs (A disintegrin and metalloproteinase) and ADAMTS (ADAM with a thrombospondin motif) are a large family of proteins involved in fertilization, neurogenesis, myogenesis and inflammatory response (reviewed in Primakoff and Myles, 2000 and Tang, 2001). The major diffrerence between ADAMs and ADAMTS is that ADAMs are membrane proteases, whereas the ADAMTs are soluble ECM proteins (Primakoff and Myles, 2000). Interestingly, only slightly more than half of the 
ADAMS/ADAMTS are functional metalloproteases due to mutations in the active site. Maybe the most important of these catalytically active proteases is tumor TNF- $\alpha$ converting enzyme (TACE/ADAM-17). It is a major inflammatory protease and several inhibitors have been developed to target it (Black, 2002). In addition, ADAMs may function as adhesion molecules through their disintegrin and cystein-rich domains. In contrast to MMPs, which have widely overlapping substrate specificities, ADAMs have much less common substrates. The ADAMs have also been implicated as binding partners with integrins. For example, ADAM-28 interacts with $\alpha_{4} \beta_{1}$ integrin (Bridges et al., 2002), ADAM-15 binds to both $\alpha_{V} \beta_{3}$ and $\alpha_{5} \beta_{1}$ integrins (Nath et al., 1999) and TACE/ADAM-17 associates with $\alpha_{5} \beta_{1}$ integrin (Bax et al., 2004). 


\section{GELATINASES IN TUMOR PROGRESSION}

Enhanced expression of MMP-2 and MMP-9 has been observed in cancers of breast, colon, lung, skin, ovary and prostate among others (reviewed by Egeblad and Werb, 2002). Increased gelatinase expression in these cancers is often accompanied with increased invasiveness and metastasis as well as decreased overall survival. Interestingly, MMP expression may be dependent on the stage of the cancer. In melanoma, increased expression of MMP-9 is found in the early steps, but at a later stage the opposite is true (van den Oord et al., 1997). In breast and colon cancer MMP-9 expression has been correlated with both increased and decreased survival and formation of distant metastasis (Pacheco et al., 1998; Scorilas et al., 2001; Takeha et al., 1997; Zeng et al., 1996). The current view is that the gelatinases and other MMPs are needed at multiple stages during the tumor progression and different tumors may utilize different MMPs. The steps where MMPs are involved include the growth of the primary tumor, angiogenesis, intravasation of the tumor cells, migration and invasion of the metastatic cells in the secondary organ as well as initiation and support of the tumor growth in the metastatic site (Figure 8). Furthermore, MMPs may either promote or suppress tumor progression by cleaving various bioactive substrates (see below). Thus, the final effect of gelatinases on tumor progression is highly context-dependent.

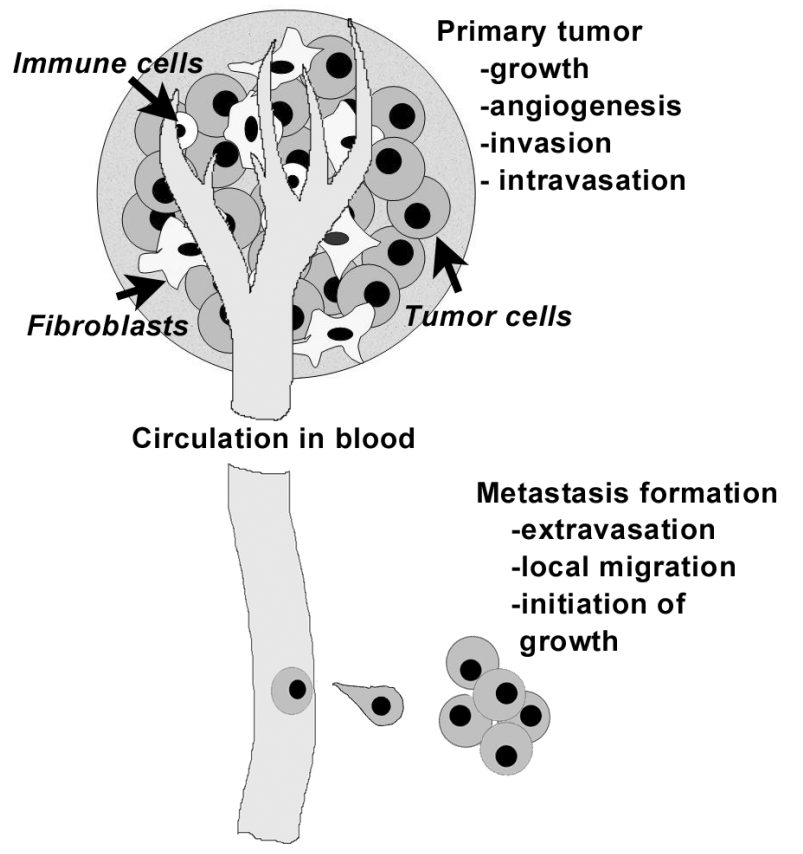

Figure 8. Schematic representation of the steps in tumor progression. Gelatinases or other MMPs have been implicated to play a role in most of these steps. Note that tumors contain in their stromal compartment nonmalignant cells such as fibroblasts and immune/inflammatory cells, which may act as a source for MMPs and other molecules to nourish the tumor development.

\section{Angiogenesis}

Angiogenesis, the formation of new blood vessels is a crucial process during the development. Angiogenesis was also realized to be a necessary step for tumor progression as without the nutrient and oxygen supply from the new blood vessels tumors ceased to grow beyond a certain size (reviewed by Folkman, 1992; Veikkola and Alitalo, 1999). This conception has yielded a myriad of strategies to inhibit tumor growth by angiogenesis modulators. These include protease inhibitors, inhibitors of growth factors and growth factor receptors, integrin inhibitors, inhibitors of signalling cascades and many other agents with undefined mechanisms (Cristofanilli et al., 2002). MMPs and especially the gelatinases are 
critically involved in angiogenesis in vitro (Schnaper et al., 1993; Seftor et al., 2001) and in vivo (Itoh et al., 1998; Vu et al., 1998). The exact mechanisms how the gelatinases contribute to angiogenesis still remain obscure and may include multiple pathways. For example, TGF- $\beta$ or phorbol ester-induced MMP-9 expression and proteolytic potential did not directly correlate with endothelial cell motility and in vitro angiogenesis (Puyraimond et al., 1999). The most direct evidence for gelatinases in angiogenesis comes from the studies of the initial steps in tumor angiogenesis. In a model of carcinogenesis of pancreatic islets in RIP1-Tag2 transgenic mice, MMP-2 and MMP-9 were found to be upregulated in angiogenic lesions. The upregulation of the gelatinases resulted in the release of bioactive VEGF, which is a major promoter of angiogenesis. Using MMP-2 and MMP-9 knockout mice, the switching from the quiescent to the angiogenic stage was found to be due to MMP-9 activity. MMP-2 deficiency did not impair the angiogenic switch but reduced the rate of tumor growth (Bergers et al., 2000). In the tumor tissue the main source of MMP-9 appeared to be the tumor-infiltrating inflammatory cells (Bergers et al., 2000; Coussens et al., 2000). However, the angiogenic switching is not solely due to to MMP-9 activity as cysteine cathepsin inhibitors also inhibit this process (Joyce et al., 2004).

In a retinal neo-vascularization model MMP-9 activity was required to expose a cryptic promigratory control site in collagen (Hangai et al., 2002). A monoclonal antibody HUIV26 recognizes an epitope in denaturated collagen type IV. Exposure of this epitope is required for angiogenesis in vivo and is associated with $\alpha_{V} \beta_{3}$ binding to collagen type IV. In contrast to retina, in melanoma vasculature the appearance of HUIV26 epitope is concomitant with the appearance of active MMP-2 (Xu et al., 2001). MMPs are also involved in developmental angiogenesis as MMP-9 and MT1-MMP deficient mice show skeletal abnormalities due to a delayed vascular invasion of the cartilage (Holmbeck et al., 1999; Vu et al., 1998; Zhou et al., 2000).

\section{Metastasis}

Metastasis is the spread of cancer cells from the primary tumor to the new metastatic sites via blood or lymph vessels (reviewed by Stacker et al., 2002). Metastasis is a highly inefficient but a deadly process (Weiss, 1990). It was long thought that some tumor cells aquire new mutations not initially present in the primary tumor cells making them metastatic. Later on, it was suggested that metastatic cells have an intrinsic signature pattern, the "poor-prognosis signature" that accounts for their metastatic behaviour. A recent study has merged these metastasis theories by showing that in addition to a poor-prognosis signature, the metastatic cells must activate additional genes, which are not activated in the primary tumor (Kang et al., 2003). Although no single gene has been identified as a major regulator of metastasis in all tumors, many animal models indicate a critical role for the MMPs, including MMP-2 and MMP-9. For example, gene expression analysis of human tumors has linked MMP-9 with a poor prognosis in breast cancer (van 't Veer et al., 2002). In an experimental metastasis assay, intravenously injected melanoma or lung carcinoma cells showed significantly decreased number of metastatic colonies in MMP-9 deficient mice (Itoh et al., 1999). These results highlight the importance of host-derived MMPs in the metastatic process. Indeed, MMP-2 and MMP-9 are often derived from the stromal cells such as fibroblasts, myofibroblasts, immune cells and endothelial cells surrounding the tumor cells, and this appears to be a common theme for most MMPs (Nelson et al., 2000; Polette et al., 1994). Other experiments have shown contribution of MMP-9 in the lung metastasis induced by VEGF receptor-1. In these experiments MMP-9 was upregulated in 
premetastatic lung endothelial cells as well as macrophages and MMP-9 deficiency led to a marked reduction in metastasis (Hiratsuka et al., 2002).

Intravasation, the invasion of the tumor cells to the blood circulation is a critical step in the metastatic process. Using the CAM assay, uPA and MMP-9, have been identified as critical players in the intravasation process (Kim et al., 1998). Intravasation was dependent on both urokinase and MMP-9, as in the absence of UPA or MMP-9, tumor cells showed only low levels of intravasation. These proteases may act in concert with the integrins as $\alpha_{V} \beta_{5}$ is also required for tumor cell dissemination in the CAM model (Brooks et al., 1997). Furthermore, $\alpha_{\mathrm{V}} \beta_{5}$, but not $\alpha_{\mathrm{V}} \beta_{3}$ mediated migration and metastasis requires growth factor-mediated tyrosine kinase signalling, such as the action of insulin-like growth factor (Filardo et al., 1995; Klemke et al., 1994), which also upregulates uPA and MMP-9 (Dunn et al., 2001; Mira et al., 1999). The proteins implicated in the metastatic process form an interesting functional loop. First, receptor bound uPA is essential for $\alpha_{V} \beta_{5}$ mediated cell migration (Yebra et al., 1996). On the other hand SPARC protein is required for $\alpha_{V} \beta_{5}$ dependent metastasis to bone (De et al., 2003). SPARC also induces MMP-1, -3 and -9 expression (Tremble et al., 1993), and the MMPs may be activated by uPA-mediated processes (Mazzieri et al., 1997; Ramos-DeSimone et al., 1999). Furthermore, SPARC can be proteolytically processed by MMPs (Sasaki et al., 1997), and the MMP-cleaved SPARC fragments modulate cell proliferation, migration and angiogenesis (Sage et al., 2003).

In contrast, extravasation, the entrance of tumor cells from the circulation to the tissues, is not critically dependent on the MMP activity. TIMP-1 overexpressing melanoma cells do not have any defect in the extravasation ability, but do show a decreased number and size of metastases after extravasation indicating a critical role for MMPs in the subsequent tumor growth (Koop et al., 1994). Similar results have also been obtained using tumor cell lines with low and high metastatic potential in mice and in the CAM assay. No difference in the extravasation rate was observed, whereas subsequent migration in the tissue and the rate of tumor cell proliferation were different (Koop et al., 1996; Morris et al., 1994). Thus whereas the spreading of the tumor cells into various organs from the primary tumor appears to be an efficient process, the subsequent growth of these metastasized cells is a rate-limiting step. Quantitative measurements on the individual steps in metastasis have confirmed that the ability of intravasation and the ability for growth expansion in the secondary organs are indeed the rate-limiting steps (Zijlstra et al., 2002). The mechanisms controlling the growth of the cancer cells in the secondary organs are not known in a detail. It is implausible that the cells would continuously proliferate in the metastatic site as it can take years before tumors can be detected in secondary organs. It thus seems that the cells either enter a state of dormancy or there is a balance of continuous proliferation and apoptosis in the micrometastasis. Both models are supported by experimental evidence. The tumor dormancy might be due to specific downregulation of cell surface molecules such as uPAR (Aguirre Ghiso et al., 1999) and angiogenic signals may then be used to terminate the dormant state (Udagawa et al., 2002). MMPs apparently also participate at this step as TIMP-1 expression by the tumor cells, but not by the surrounding tissue, significantly affects the initiation and growth of the tumors (Soloway et al., 1996). There are also evidence supporting the continuos proliferation/apoptosis model of metastases (Barnhill et al., 1998). 


\section{Cancer-associated inflammation}

It has been estimated that over $15 \%$ of the malignancies have an infectious origin (Kuper et al., 2000). In addition to these, the leukocytes participate in the tumor progression also in the absence of infectious agents. Chronic inflammation associated with some cancers can further stimulate cancer progression due to the release of MMPs from the inflammatory cells (Coussens et al., 1999; Coussens et al., 2000). The critical role of MMP-9, apparently derived from leukocytes, in the initiation of angiogenesis was discussed above. Tumor cells produce an array of cytokines and chemokines that induce leukocyte infiltration to the tumor. The same cytokines and chemokines may also promote tumor growth by an autocrine mechanism. The tumor-infiltrated leukocytes, including neutrophils, dendritic cells, macrophages, eosinophils, mast cells and lymphocytes can extensively modify tumor microenvironment by producing additional cytokines, reactive oxygen species, proteases including MMPs, interferons and other compounds (Coussens and Werb, 2002). However, inflammation in cancer is a two-bladed sword as the compounds relased by the inflammatory cells may either suppress or enhance tumor progression. Thus, it appears that a delicate balance of pro- and anti-tumor activity defines the fate of the tumors; both excessive and inadequate exploitation of the inflammatory components produced by leukocytes and the other stromal cells is detrimental to the tumor growth (Coussens and Werb, 2002).

Chemokines are important mediators of leukocyte recruitment into the tumors. In addition to their role in the regulation of directional migration of leukocytes, chemokines are also able to directly modify endothelial and tumor cell chemotaxis and thus affect migratory and invasive behaviour of the tumor. Some chemokines may inhibit directly or indirectly angiogenesis, whereas others are proangiogenic. Furthermore, chemokines may be proteolytically processed to regulate their activity. For example, monokine induced by interferon IFN- $\gamma$ (MIG), platelet factor (PF)-4, interferon-inducible protein-10 (IP10/CXCL-10) and stromal-cell derived factor (SDF)-1 are angiostatic chemokines (Moore et al., 1998), which can be cleaved by the gelatinases thus potentially enhancing angiogenesis. Similarly, the gelatinases can process angiogenic chemokines including granulocyte chemotactic protein (GCP)-2, epithelial-cell derived neutrophil activating peptide (ENA)78, growth-regulated oncogene (GRO)- $\alpha$ and pro-interleukin-8 (Moore et al., 1998) (see Table 1). Some chemokines also participate in the homing of tumor cells into the metastatic sites (Muller et al., 2001; Nathanson, 2003). Hence, the gelatinases may affect metastasis also by regulating chemokine activity. The gelatinases, especially MMP-9, have been implicated in the negative regulation of immune response to cancer by cleaving the interleukin- $2 \alpha$ receptor, activation of TGF- $\beta$ and by shedding of ICAM-1 (Fiore et al., 2002; Sheu et al., 2001; Yu and Stamenkovic, 2000). MMP-2 is able to proteolytically process monocyte chemoattractant proteins and suppress inflammation in vivo (McQuibban et al., 2002).

As the action of inflammatory cells is intimately involved in tumor progression, the use of anti-inflammatory agents, such as the cyclo-oxygenase inhibitors is an attractive approach for anti-cancer therapy (Liu et al., 2002; Williams et al., 1999). Another possibility could be the prevention of leukocyte migration into the tumors. The selective expression of $\beta_{2}$ integrins in leukocytes might provide a suitable therapeutic window, although leukocytes may also utilize other integrins for migration (Worthylake and Burridge, 2001). 


\section{Dual role of gelatinases in cancer}

Although inhibition of gelatinases in many cases results in regression of tumor growth, the gelatinases, especially MMP-9 can also stimulate tumor growth. Based on the data obtained with MMP and TIMP knockout animals, as well as with various in vitro models and clinical trials, it has become evident that gelatinases and other MMPs have both pro- and antiangiogenic properties. Furthermore, it has become evident that there are tissue- and tumor specific differences in the use of MMPs and TIMPs (reviewed by Egeblad and Werb, 2002). The dual role of MMPs in cancer is somewhat similar to the role of $\alpha_{\mathrm{V}}$ integrins. The $\alpha_{\mathrm{V}}$ integrin antagonists effectively suppress tumor angiogenesis in in vitro and in animal models (Brooks et al., 1994; Friedlander et al., 1995; Hammes et al., 1996). However, the knockout mice of $\alpha_{V} \beta_{3}$ and $\alpha_{V} \beta_{5}$ alone or in combination show increased angiogenesis and tumor growth (Hynes, 2002; Reynolds et al., 2002). Although genetic ablation of all $\alpha_{\mathrm{V}}$ integins is detrimental in mice, some of the pups were born alive. Extensive vasculogenesis was also observed implicating that these integrins are not the sole regulators of blood vessel formation (Bader, 1998).

One of the anti-angiogenic activities of the MMPs, is the generation of angiostatin, a proteolytic fragment of plasminogen. As it name implies it is a negative regulator of angiogenesis. Of the MMPs, MMP-2, $-7,-9$ and -12 can generate angiostatin. This may be a crucial factor in controlling the growth rate of certain tumors. Tumors grown in integrin $\alpha_{1}$ knockout mice were significantly smaller than those grown in wild type mice, these tumors also showed remarkably reduced angiogenesis and increased plasma angiostatin. In further experiments elevated levels of MMP-7 and MMP-9 were found to be responsible for angiostatin generation (Pozzi et al., 2000) and low plasma levels of MMP-9 were associated with increased angiogenesis (Pozzi et al., 2002). A similar effect would propably be observed by MMP-9-generated endostatin (Ferreras et al., 2000). A third anti-angiogenic protein generated by MMP-9 is tumstatin, a fragment from the noncollagenous domain of collagen type IV $\alpha 3$ chain. Interestingly, physiological concentrations of tumstatin effectively suppress tumor growth. MMP-9 deficient mice showed an accelerated rate of tumor growth after an initial lag period. This increased tumor growth was linked to the absence of tumstatin (Hamano et al., 2003). Tumstatin knockout mice similarly showed increased tumor growth associated with enhanced tumor angiogenesis. Interestingly, angiogenesis associated with the normal development and tissue repair was normal (Hamano et al., 2003). As discussed above, proteolytic processing of chemokines may be an additional level where the gelatinases may either suppress or enhance tumor progression. It is currently unclear to what extent this activity of gelatinases affects tumor growth. Other MMPs, however, have been implicated to play a role in the regulation of tumor-associated inflammation. MMP-8 deficient mice show an increased incidence of skin tumors in a chemical carcinogenesis model (Balbin et al., 2003). These mice also developed the tumors more rapidly than the wild-type mice and the observed effect could be tracked to a sustained inflammatory response in the tumor (Balbin et al., 2003). 


\section{THERAPEUTIC POSSIBILITIES WITH THE MMP INHIBITORS}

Despite the beneficial effects of MMP inhibitors in the in vitro assays and in animal models, most clinical trials so far have been disappointing. The reasons for these failures have been widely analyzed (Coussens et al., 2002; Hidalgo and Eckhardt, 2001; Overall and LopezOtin, 2002; Pavlaki and Zucker, 2003). The main reason for the failures of MMP-inhibitors is that most clinical studies involved advanced stage cancers. It has now been established that the MMP-inhibitors such as marimastat are not particularly effective at the later stages as also shown with the mouse models (Bergers et al., 1999). Another reason for the failures is that no reliable biomarkers have been available for the evaluation of the efficacy of MMP inhibitors. Thus it has been utmost difficult or impossible to show that the inhibitors would have sufficiently inhibited their targets (Coussens et al., 2002). For example, analysis of the serum or plasma levels of MMPs has been largely uniformative (Hidalgo and Eckhardt, 2001), but the recently developed MMP-activity imaging agents may alleviate this problem (Bremer et al., 2001). A further difficulty in the evaluation of MMP-inhibitor activity in the clinical trials is that the MMP inhibitors are not cytotoxic. Thus they do not directly cause shrinkage of the established tumors. This requires setting of new endpoints in the evaluation of drug efficacy.

Moreover, the first clinical studies were initiated when there was only a limited knowledge on the role of various MMPs in cancer and they utilized broad-spectrum inhibitors of MMPs with little or no specificity towards individual proteases. Whether the more selective inhibitors will show any more benefit in the clinical trials is still a major question, as even a single MMP can have both pro- and anti-tumor activities. In accordance with this, the observations that some MMP-deficient mice show increased tumor growth, is a major concern (Balbin et al., 2003; Coussens et al., 2000; Hamano et al., 2003). Thus alternative and/or more selective MMP-inhibition strategies are likely to be required, but even their success cannot be guaranteed.

Despite the poor success of most MMP inhibitor trials, important data has been accumulated supporting the development of MMP inhibitors as therapeutic agents. Phase I studies, which aim to evaluate the safety of the tested drug-candidates have established that the MMP inhibitors are in general safe and well tolerated. The main adverse effect of MMP-inhibitors has been musculoskeletal pain (Rosemurgy et al., 1999). The effect is propably due to inhibition of ADAMs, as those MMP inhibitors with a reduced activity to the ADAMs do not show these side-effects (Brown, 2000). Furthermore, not all trials have been failures. Marimastat has shown modest increase in overall survival of patients with gastric cancer and pancreatic cancer (Bramhall et al., 2002; Pavlaki and Zucker, 2003).

MMP inhibitors are best viewed as anti-angiogenic agents for primary tumors and as agents to prevent metastasis. Before attempting cancer therapy with the MMP inhibitors, it would be beneficial to measure MMPs in tumors and blood as a means of identifying those who are likely to respond to the therapy (Zucker et al., 2000). However, as discussed above, the plasma levels of MMPs may not be sufficiently informative for this purpose. A combination therapy of MMP-inhibitor together with a cytotoxic drug seems to be a more attractive alternative. Some reports have indicated a synergistic effect of gelatinase selective inhibitors with apoptosis inducing agents in cell culture (Nyormoi et al., 2003). Animal models with a combination therapy have been promising, with reduced metastasis and increased survival (Haq et al., 2000; Liu et al., 2003; Yamamoto et al., 2003). As a consequence, several clinical studies with combination therapy are now in progress (Hoekstra et al., 2001; Nelson 
et al., 2000), but the first failures with the combination therapy have been already reported (Bramhall et al., 2002).

Several strategies have been suggested for the future MMP inhibitor trial development (Pavlaki and Zucker, 2003). These include inhibitors that can be administred for long periods of time without side-effects, combination of MMP inhibitors with other proteinase inhibitors, and inhibition of MMP synthesis, in addition to aforementioned design of more selective inhibitors and treating cancer at earlier stages. Alternatively, inhibition of localized MMP activity may be a potential inhibition approach as demonstrated by the inhibition of MMP-2 binding to the $\alpha_{V} \beta_{3}$ integrin without inhibition of MMP-2 activity (Silletti et al., 2001). The overexpression of MMPs in cancer provides also alternative therapeutic approaches, independent on MMP inhibition. Targeting of drugs encapsulated into liposomes coated with MMP-targeting compounds is one possibility. Another novel use of MMPs is to use prodrugs that become cytotoxic upon proteolytic cleavage by an MMP (Kratz et al., 2001; Mansour et al., 2003) or MMP-activatable gene therapy vectors (Peng et al., 1997). As it is now evident that MMPs promote cancer progression mainly at the early stages, it is crucial to identify the primary tumors as early as possible. The MMP inhibitors coupled to imaging agents could be used to detect small tumors due to their tumor-targeting properties (Bremer et al., 2001; Li and Anderson, 2003; Medina et al., 2001).

Finally, if cancer remains refractory to the treatment with the MMP inhibitors even after novel treatment strategies, various inflammatory, degenerative and vascular diseases could be other disease targets for these inhibitors. 


\begin{abstract}
AIMS OF THIS STUDY
It has recently become evident that despite the widely observed association of gelatinase activity with the invasive capacity of cancer cells, the active site inhibitors of MMPs do not show beneficial effects in the treatment of human cancers. This is at least partially due to the fact that the exact biological functions of these enzymes are not fully understood. Some MMPs have been shown to bind cell surface receptors, such as integrins and CD44, and these interactions appear to play an important role in the regulation of MMP functions. To gain more insight into the functions of the gelatinases in cell migration and invasion, I have utilized phage display to unravel molecular details about the cell-surface interactions of MMP-9. Phage display is a powerful tool to identify peptides that bind to proteins and other biomolecules. The major advantage of phage display is that it offers unique information about protein-protein interactions and simultaneously provides novel means to inhibit these. However, the inherent proteinaceous nature of the identified peptide ligands may limit their utility in drug development. With these issues in mind I have focused on the identification and development of novel inhibitors of gelatinase-mediated cancer cell migration and invasion, and extended the utility of phage display technology. During the course of the work, the following questions were raised:
\end{abstract}

1) How could phage display peptides be analysed more conveniently for activity and solubility properties, and could the activity and stability of the peptides be easily improved to extend the utility of these compounds?

2) Which integrins, if any, act as receptors for MMP-9 on the surface of leukemia and fibrosarcoma cells, and what is the mechanism of binding? Are there any additional cell-surface interactions?

3) Is prevention of cell-surface association of MMP-9 a feasible way to inhibit cancer cell migration and invasion?

4) Could the peptides displayed on a phage be directly used to identify potent small-molecule compounds, which would better suit for drug development? 


\section{MATERIAL AND METHODS}

A summary of the methods used will be given. Detailed methods can be found in the original publications.

Standard phage display. Phage display selections were made using a pool of random peptide libraries $\mathrm{CX}_{7-10} \mathrm{C}$ and $\mathrm{X}_{9-10}$ (Koivunen et al., 1999). The target proteins were immobilized on microtiter wells at a $2 \mu \mathrm{g} / \mathrm{ml}$ concentration. The phage library pool was added to the wells with or without a subtractive step with GST-coated wells. After three rounds of selection the phage sequences were determined by amplifying the peptide encoding sequences using primers F1-F 5'-TAA TAC GAC TCA CTA TAG GGC AAG CTG ATA AAC CGA TAC AAT-3' and F1-R 5'-CCC TCA TAG TTA GCG TAA CGA TCT-3'. The PCR products were sequenced using the primer F1-S 5'-TAA ACC GAT ACA ATT AAA GGC TC-3'. The phage binding specificity was tested with immobilized proteins ( $20 \mathrm{ng} /$ well). The phage $\left(10^{8}\right.$ transducing units/well) were allowed to bind in the absence or presence of competitor peptides $(20 \mu \mathrm{M})$ followed by washings with PBS- $0.05 \%$ Tween 20 (PBST). The bound phage were detected with peroxidase-conjugated anti-phage antibody (Amersham Biosciences).

Phage display with amino acid analogues. The fluorophage library was prepared from 15 $\mu 1$ aliquots of $\mathrm{CX}_{7} \mathrm{C}$ and $\mathrm{CX}_{8} \mathrm{C}$ libraries infected to tryptophan auxotrophic MB5F strain. The phage-infected bacteria were transferred to a mimimal medium and cultured in the presence of 5-fluorotryptophan and 6-fluorotryptophan. The fluorophage library (a $20 \mu 1$ aliquot) was added to the protein coated wells in $50 \mathrm{mM}$ Tris- $\mathrm{HCl}, 150 \mathrm{mM} \mathrm{NaCl}$ (pH 7.5) containing 1\% BSA. After extensive washings the bound phage were eluted and used to infect the MB5F strain. The infected bacteria were cultured overnight, transferred to M9 medium containing tryptophan and again cultured overnight. The bacteria were transferred into $100 \mathrm{ml}$ M9 medium with tryptophan and cultured until OD600 was 0.7-1.0. The bacteria were then resuspended into $100 \mathrm{ml}$ of $\mathrm{M} 9$ medium containing $0.5 \mathrm{mM}$ (as Lisomer) $5 \mathrm{FW}$ and $6 \mathrm{FW}$ and cultured overnight. The phage were collected using $\mathrm{PEG} / \mathrm{NaCl}$ precipitation.

Preparation of auxotrophic bacterial strains. In vitro assembled bacteriophage Mu DNA transposition complexes were used to mutagenize the parental bacterial strains (Lamberg et al., 2002). The transposition complexes were electroporated into E. coli ER2566 or MC1061 strains. Successful transpositions were identified by gain of antibiotic resistance. The clones obtained were replica-plated on M9 minimal plates and M9 plates containing the amino acid to be screened for auxotrophism. The final strains ER2566/Trp82 and MB5F are auxotrophic for tryptophan and the MB64F is auxotrophic for methionine.

Peptide biosynthesis. Recombinant peptides were cloned using oligonucleotides coding for the desired peptide or by PCR amplification from the peptide bearing phage using universal primers. The sequences for the universal primers are 5'CCTTTCTGCTCTTCCAACGCCGACGGGGCT-3' ${ }^{\prime}$ and 5'ACTTTCAACCTGCAGTTACCCAGCGGCCCC-3'. The insert was digested with SapI and PstI and ligated into similarly digested pTwin vector (New England Biolabs). The presence of the correct insert was verified by DNA sequencing. The plasmid encoding the intein-peptide fusion was transformed into $E$. coli ER2566 strain. The bacteria were cultured in LB medium containing $100 \mu \mathrm{g} / \mathrm{ml}$ ampicillin and protein expression induced with IPTG $(0.3 \mathrm{mM})$ The intein-peptide fusions were purified with chitin affinity chromatography. The 
intein-cleavage reaction was performed on-column by overnight incubation in $\mathrm{pH} 7.0$ buffer at room temperature. The free peptide was eluted, concentrated and purified with reversephase HPLC. The identity of each peptide was verified by mass spectrometry. Peptides were quantified using $o$-phthalaldehyde or HPLC analysis. Tryptophan analogue containing peptides were expressed in the tryptophan auxotrophic ER2566/Trp82 in a minimal medium in the presence of 5-hydroxy-L-tryptophan, 5-fluoro-DL-tryptophan, 6-fluoro-DLtryptophan or DL-7-azatryptophan.

Cell culture. Human HT1080 fibrosarcoma cells, human monocytic leukemia THP-1 cells, human acute myeloid leukemia OCI-AML-3, human histiocytic lymphoma U937 cells, human tongue squamous cell carcinoma cell line HSC-3, hamster melanoma cell line CS-1 and the $\beta_{5}$ integrin transfected derivative were maintained in DMEM or RPMI supplemented with 10\% FBS, L-glutamine, penicillin and streptomycin.

Cell adhesion, migration and invasion. Cell adhesion was studied by coating microtiter wells with various peptides or proteins. The cells were stimulated with $50 \mathrm{nM} \mathrm{PDBu}$ to activate the $\beta_{2}$ integrins. Nonadherent cells were removed by gentle washing with PBS or in some cases with $2.5 \mathrm{mM}$ EDTA in PBS and the adherent cells quantitated by measuring intracellular phosphatase activity using $p$-nitrophenyl phosphate as a substrate (Koivunen et al., 2001). Alternatively, THP-1 cells were stimulated with $20 \mathrm{nM} \mathrm{PDBu}$ and allowed to adhere on uncoated plastic overnight. Nonadherent cells were removed by washing with PBS followed by additional washes with $2.5 \mathrm{mM}$ EDTA in PBS. Migration of HT1080, THP-1 and OCI-AML-3 cells was studied using transwells coated with various proteins. The cells (20 000-50 000/100 $\mu$, depending on the experiment) were allowed to migrate in the presence of $10 \%$ serum containing culture medium. Invasion assays were conducted using Matrigel coated transwells (BD Biosciences). The cells migrated to the underside of the membrane were stained with crystal violet and counted under light microscope (Koivunen et al., 1999).

Activation of the gelatinases. In vitro activation of MMP-2 was done with APMA and MMP-9 with trypsin or MMP-3. For the activation of MMP-9, THP-1 cells (40 000/100 $\mu 1)$ or confluent HT1080 cells were incubated for $16 \mathrm{~h}$ in the presence or absence of $2.5 \mu \mathrm{g} / \mathrm{ml}$ plasminogen, $0.5 \mu \mathrm{g} / \mathrm{ml}$ pro-MMP-3, $40 \mathrm{nM} \mathrm{PDBu}$, and the peptides at a $200 \mu \mathrm{M}$ concentration unless otherwise indicated. Aliquots of the conditioned media were analyzed by gelatin zymography.

Gelatinase activity assays. Gelatinase inhibitory activity was determined using either one of the three assays: 1) The degradation of biotinylated gelatin was examined using a gelatinase activity kit according to the manufacturer's instructions (Roche). 2) The degradation of a MMP-2 specific fluorescent peptide substrate MCA-Pro-Leu-Ala-NvaDpa-Ala-Arg- $\mathrm{NH}_{2}$ (Calbiochem, $2.5 \mu \mathrm{M}$ final concentration) was analyzed using a MOS250 spectrofluorometer (Bio-Logic SA, Claix, France) with $330 \mathrm{~nm}$ excitation and $390 \mathrm{~nm}$ emission. 3) The degradation of $\beta$-casein was studied by incubating MMP- 2 with $0.1 \mathrm{mg} / \mathrm{ml}$ concentration of $\beta$-casein for $2 \mathrm{~h}$ at $37^{\circ} \mathrm{C}$ followed by analysis by SDS-PAGE.

Zymography. Gelatinases in the conditioned medium of the cultured cells were detected using gelatin co-polymerized in SDS-PAGE gels (Hibbs et al., 1985). Briefly, the samples were run in non-reducing conditions followed by washing of the gels to remove SDS and allow refolding of the gelatinases. The gels were then incubated in the gelatinase activity buffer for 16 hours followed by staining of the gels with coomassie brilliant blue. The 
gelatinase activity is visualized as white bands on a uniform blue background. uPA activity was detected using plasminogen/casein zymography as described (Myöhänen et al., 2001).

Expression of recombinant MMP and integrin domains. The following MMP constructs were cloned and expressed for this study. The collagen binding domain of MMP-9 $\left(\mathrm{Gly}^{204}\right.$ $\mathrm{Gly}^{373}$ ), the C-terminal domain of MMP-2 $\left(\mathrm{Glu}^{438}-\mathrm{Cys}^{631}\right)$ and MMP-9 (Asp ${ }^{494}-\mathrm{Asp}^{688}$ ) and proMMP-9 with the hinge region and C-terminus deleted (proMMP-9- $\Delta \mathrm{HC}, \mathrm{Ala}^{1}-\mathrm{Gly}^{424}$ ). These proteins were expressed in $E$. coli and purified using gelatin affinity or $\mathrm{Ni}^{2+}$-chelating affinity chromatography. The integrin $\beta_{5}$ I-EGF2+3 fragment $\left(\mathrm{Glu}^{476}-\mathrm{Asn}^{563}\right)$ was cloned from $\beta_{5}$ cDNA and expressed with an N-terminal His6-tag. Single point mutations to MMP$9, \beta_{5}$ I-EGF2+3 fragment and the $\alpha_{M}$ I domain were prepared by site directed mutagenesis.

ProMMP-9 and gelatin binding to leukocyte $\alpha_{M}$ integrin. ProMMP-9 binding to the $\alpha_{M} I$ domain in the presence of peptides was studied as described by immobilizing the I domain on microtiter wells and detecting bound MMP-9 with a monoclonal antibody to MMP-9 (clone GE-213, NeoMarkers). ProMMP-9 was competed with peptides or chemicals. Gelatin binding to the proMMP-9/ $\alpha_{\mathrm{M}} \beta_{2}$ integrin complex was studied by immobilizing the integrin $\alpha_{M} \beta_{2}$ or $\alpha_{\mathrm{IIb}} \beta_{3}$ as a control. ProMMP-9 was incubated for $2 \mathrm{~h}$ and the unbound proMMP-9 was washed away. Biotinylated gelatin was allowed to bind and was detected with streptavidin-peroxidase. The $\alpha_{M}$ I domain binding to proMMP-9 was also studied by immobilizing the MMP. Briefly, the catalytically inactive proMMP-9- $\Delta \mathrm{HC}-\mathrm{E}^{402} \mathrm{Q}$ mutant was prepared via site-directed mutagenesis from the wild-type proMMP-9- $\Delta \mathrm{HC}$ and coated on microtiter wells. Soluble $\alpha_{M}$ integrin I domain-GST fusion $(2.5 \mu \mathrm{g} / \mathrm{ml})$ was added in the presence or absence of peptides or compounds and incubated for one hour. Bound GST fusion was detected with anti-GST antibody and peroxidase-conjugated anti-goat antibody.

Gelatin and CBD binding assays. Recombinant CBD or human plasma fibronectin (Calbiochem) was immobilized in microtiter wells. The wells were saturated with 1\% BSAPBST. Biotinylated gelatin was added with or without the peptides at the concentrations indicated or with an excess of unlabelled gelatin and allowed to bind for $1 \mathrm{~h}$. Bound gelatin was detected with streptavidin-peroxidase. CBD binding to immobilized fibronectin, the $110-\mathrm{kDa}$ fragment of fibronectin (Upstate Biotechnology) or urea-denaturated human plasma vitronectin $(1 \mu \mathrm{g} /$ well $)$ was studied using biotinylated CBD $(5 \mu \mathrm{g} / \mathrm{ml})$ in $1 \%$ BSAPBST in the presence or absence of $20 \mu \mathrm{M}$ peptides.

Immunoprecipitation and western blotting. HT1080 cells were treated with $50 \mathrm{nM}$ PDBu for $3 \mathrm{~h}$ in serum-free medium, washed with PBS and lysed in $10 \mathrm{mM}$ Tris- $\mathrm{HCl}(\mathrm{pH} \mathrm{8.0)/140}$ $\mathrm{mM} \mathrm{NaCl} / 1 \%$ Triton X-100/1 mM PMSF followed by immunoprecipitation with anti-uPAR (399R, American Diagnostica, Greenwich, CT) or anti-MMP-9 (H-129, SantaCruz Biotechnology) or a control IgG. Integrins were immunoprecipitated with chain-spesific anti-integrin cytoplasmic domain antisera. The immunoprecipitates were resolved on an $8 \%$ SDS-PAGE gel, blotted and detected with anti-MMP-9 antibodies.

Immunofluorescence. HT1080 cells were allowed to adhere on vitronectin $(10 \mu \mathrm{g} / \mathrm{ml})$ in serum-free DMEM. Directional migration of the cells was stimulated by overlaying the cells with $0.5 \%$ agarose in DMEM and adding $5 \mu \mathrm{FBS}$ with PDBu (20 nM final concentration) to the one end of the wells. Cells were cultured overnight, washed with PBS, fixed with paraformaldehyde, permeabilized, and stained with the monoclonal anti-uPAR antibody (Ab3937, American Diagnostica) or anti- $\beta 5$ integrin IA9 and polyclonal MMP-9 antibodies (H-129). 
Pericellular proteolysis. Microtiter wells were coated with a mixture of fibronectin (10 $\mu \mathrm{g} / \mathrm{ml})$ and FITC-labelled gelatin $(100 \mu \mathrm{g} / \mathrm{ml})$ followed by saturation with $1 \%$ BSA in PBS. HT1080 cells (50 000 in $100 \mu 10.1 \% \mathrm{BSA} / \mathrm{DMEM})$ were incubated in the presence of 20 $\mathrm{nM}$ PDBu and the peptides or the MMP-2/MMP-9 selective inhibitor InhI (Calbiochem). As a control non-activated cells and medium without the cells were used. Gelatinolysis was measured as the increase of fluorescence from an aliquot of the conditioned medium.

uPAR clevage. Recombinant soluble human uPAR (suPAR, R\&D Systems) was digested with trypsin-activated MMP-9 in the presence of $10 \mu \mathrm{g} / \mathrm{ml}$ aprotinin with or without $10 \mathrm{mM}$ EDTA. The samples were incubated for 16 hours in $37^{\circ} \mathrm{C}$ and analyzed by western blotting with anti-uPAR antibodies. uPAR cleavage on the surface of HT1080 cells, THP-1 or OCIAML-3 cells was studied in a serum-free medium with or without $20 \mathrm{nM}$ PDBu for $48 \mathrm{~h}$ in the presence or absence of inhibitors. The cells were washed with PBS, incubated with 50 $\mathrm{mM}$ glycine- $\mathrm{HCl}(\mathrm{pH} 3.0) / 100 \mathrm{mM} \mathrm{NaCl}$ to extract cell surface bound urokinaseplasminogen activator (uPA) and MMPs, and neutralized with $500 \mathrm{mM}$ Hepes (pH 7.5)/100 $\mathrm{mM} \mathrm{NaCl}$. Membrane proteins were enriched by Triton X-114 phase separation and analyzed by western blotting with antibodies to uPAR.

${ }^{125}$ I-C domain binding to CS-1 melanoma cells. The MMP-9 C domain was labelled with ${ }^{125} \mathrm{I}$ to a specific activity of $0.06 \mu \mathrm{Ci} / \mathrm{pmol}$. The labelled domain retained $40 \%$ of the $\mathrm{CRV}$ peptide-binding activity as shown by the phage-binding assay. The CS-1 cells were washed with $2.5 \mathrm{mM}$ EDTA in PBS and suspended in $20 \mathrm{mM}$ Hepes $(\mathrm{pH} 7.5) / 150 \mathrm{mM} \mathrm{NaCl} / 1 \mathrm{mM}$ $\mathrm{MnCl} 2 / 0.2 \mathrm{mM} \mathrm{CaCl} 2 / 0.5 \% \mathrm{BSA}$. After a preincubation of $1.5 \times 10^{6}$ cells on ice for $30 \mathrm{~min}$ with the competitors, ${ }^{125}$ I-labelled $\mathrm{C}$ domain $\left(1 \times 10^{6} \mathrm{cpm}\right)$ was added and incubated for three hours on ice. The cells were transferred to tubes containing $200 \mu 1$ of dibutyl phthalate/cyclohexane mixture $(23: 2 \mathrm{vol} / \mathrm{vol})$, centrifuged $7500 \mathrm{xg}$ for 10 minutes and snapfrozen. The bottom of the tube containing the cells were cut and analyzed with a gammacounter.

Human tumor xenograft growth in vivo. The animal studies were approved by the ethical committee of Helsinki University. HSC-3 tumors were established by administering $5 \times 10^{6}$ tumor cells in PBS in both flanks of the Hsd:Athymic Nude-nu mice. After three days, the mice received five daily injection of $0.8 \mathrm{mg} / \mathrm{ml} \mathrm{CRV} \mathrm{or} \mathrm{the} \mathrm{scrambled} \mathrm{peptide} \mathrm{or} \mathrm{the} \mathrm{vehicle}$ (PBS) in a $200 \mu \mathrm{l}$ volume. Three-dimensional caliper measurements were taken twice a week and the tumor volumes calculated. Mice were sacrificed when the tumor volume reached $1000 \mathrm{~mm}^{3}$. For the staining of the tumor vasculature, frozen tissue sections were stained with anti-CD31 antibody (MEC 13.3, BD Biosciences).

Small molecule compound library screening. A combinatorial library of 10000 small molecules was purchased from ChemBridge (San Diego, CA). A competition assay with the DDGW peptide bearing phage was set up by immobilizing $20 \mathrm{ng} / \mathrm{well}$ recombinant $\alpha_{\mathrm{M}} \mathrm{I}$ domain-GST fusion in 96-well plates. The compounds were used in pools comprising eight compounds, each at a $5 \mu \mathrm{M}$ concentration and DMSO at a $1.25 \%$ concentration. After preincubation of the compounds in the wells, DDGW phage was added $\left(3 \times 10^{8}\right.$ transducing units/well). Phage binding was detected with an anti-phage antibody and pools with inhibitory activity were re-tested as single compounds. 


\section{RESULTS AND DISCUSSION}

Brief descriptions of the main results are given together with additional views that are not emphasized in the original publications due to space limitations. Detailed discussions are found in the original publications.

\section{Biosynthesis of phage display peptides (I, III-IV).}

Inteins were examined as a fusion partner for phage display peptide expression in E. coli. A $\mathrm{pH} /$ temperature cleavable intein was used to obtain cyclic disulfide-bonded peptides, thus avoiding the use of thiol compounds. As a proof of principle, the gelatinase inhibitor peptide CTTHWGFTLC (CTT) was cloned and expressed. Synthetic and recombinant CTT peptides were identical in all gelatinase inhibition assays thus validating the intein expression strategy for the phage display peptides. Both peptides inhibited MMP-2 with an $\mathrm{IC}_{50}$ of 20 $\mu \mathrm{M}$. The significance of the intein expression system is that is allows rapid preparation of soluble peptides after a phage display selection. This markedly helps the analysis of phage binding specificity and allows initial characterization of the usefulness of the selected peptides. We also prepared recombinant single alanine mutants from the CTT peptide to study which amino acid residues are critical for the gelatinase inhibitory activity. The central amino acids Trp, Gly and Phe were found to be the most critical, whereas other mutations reduced the activity less than $20 \%$. With the knowledge that tryptophan is the most essential residue for gelatinase activity we aimed at replacing this residue with unnatural analogues in order to improve the activity of the CTT peptide. We further designed universal primers, which allow direct PCR-based cloning of phage-displayed peptides into the intein vector. These primers incorporate ADGA and GAAG peptide sequence into the $\mathrm{N}$ - and the $\mathrm{C}$-terminus of the expressed peptide, respectively. The universal primers were used in subsequent phage display selections where the DDGW, PPC and CRV peptides were identified.

\section{Increase of serum stability of gelatinase inhibitor peptide CTT by incorporation of a 5-fluorotryptophan (I)}

Unnatural amino acids have not been previously incorporated into recombinant peptides. We isolated a tryptophan auxotrophic mutant $E$. coli from a library of MuA transposition mutagenized bacteria. With this bacterial strain designated ER2566/Trp82 and the intein expression system, we were able to obtain peptides containing 5- and 6-fluorotryptophan and 5-hydroxytryptophan. None of these modified peptides showed a better gelatinase inhibitory activity than the parental CTT peptide. As the analogue containing peptides may be proteolytically more stable than peptides containing only normal amino acids, we studied the stability of the peptides in human serum by immunoblotting. Unfortunately, only the stability of the 5F-Trp CTT could be analyzed as the other analogue containing peptides were not recognized by an anti-CTT peptide antibody. The 5F-Trp CTT peptide was found to be 6-fold more stable than the parental peptide. It was further found to be a more potent inhibitor of HT1080 fibrosarcoma cell invasion in normal human serum, but not in heatinactivated fetal bovine serum, apparently due to the increased serum stability. In this assay, the 6F-Trp CTT was also included, but did not show any beneficial effect compared to the parental CTT peptide. 


\section{Incorporation of amino acid analogues into phage display libraries (II)}

We reasoned that it would be even more advantageous to incorporate amino acid analogues into phage display libraries than into preselected peptides. This would allow direct isolation of peptides with improved activity and/or stability. We thus isolated auxotrophic mutants of E. coli MC1061. This strain was selected as it is commonly used for phage library preparation and also as it is naturally auxotrophic for leucine. The isolated tryptophan and methionine auxotrophs were further modified by adding a $\mathrm{F}$ pilus resulting in strains designated MB5F and MB64F, respectively. Formation of infective phage particles in the presence of various amino acid analogues was then studied. The fluorinated tryptophan analogues well supported the phage production. Norleucine, a methionine analogue, was also well incorporated and supported the production of infective phage particles. Leucine analogue incorporation was unsuccessful. The incorporation of tryptophan analogues was directly confirmed by the distinct fluorescence emission spectra of the fluorotryptophan containing phage particles.

We prepared a 5F-Trp and 6F-Trp containing peptide library by infecting our standard $\mathrm{CX}_{7} \mathrm{C}$ and $\mathrm{CX}_{8} \mathrm{C}$ libraries into the $\mathrm{MB} 5 \mathrm{~F}$ strain. The resulting library now containing an extended amino acid repertoire was used in a biopanning with MMP-2 and MMP-9 Cterminal domains. After three rounds of selection with the fluorophage library, the MMP-9 C domain showed a 19-fold enrichment compared to the BSA control. Out of nine peptides, there were four containing a tryptophan residue, thus a potential fluorotryptophan. Although we were successful in the incorporation of amino acid analogues, this method is still in its infancy. A limiting factor is that only some amino acid analogues are incorporated thus constraining the diversity of the libraries that may be constructed. However, even a single amino acid analogue may significantly change the properties of the polypeptides (Hsieh et al., 1987; Tang et al., 2001; Tang and Tirrell, 2001). To further improve the system, the phage infectivity of the auxotrophic hosts should also be increased. Despite these limitations, this concept offers important advantages for the development of more diverse peptide libraries.

\section{Inhibition of MMP-9 interaction with $\alpha_{M}$ integrin I domain by phage display peptides containg a DDGW motif (III)}

It has been previously identified that proMMP-1 binds to the $\alpha_{2}$ integrin I domain (Dumin et $a l ., 2001)$ and that the binding involves the hemopexin-like C-terminal domain and the adjacent linker region in MMP-1 (Stricker et al., 2001). We thus investigated if leukocyte integrin I domains could bind MMPs. Furthermore, we were interested in developing peptide inhibitors for such an interaction. Biopanning with $\alpha_{M}$ integrin I domain identified a predominant peptide motif with $(\mathrm{D} / \mathrm{E})(\mathrm{D} / \mathrm{E})(\mathrm{G} / \mathrm{L}) \mathrm{W}$ sequence. Similarity search to this motif identified that many MMPs have a related, well-conserved sequence present in their catalytic domain, MMP-2 and MMP-9 showing the best matches with the phage sequences. The suspected binding site in the catalytic domain was further confirmed in a pepspot membrane assay spanning the whole proMMP-9 sequence. The peptide containing the $(\mathrm{D} / \mathrm{E})(\mathrm{D} / \mathrm{E})(\mathrm{G} / \mathrm{L}) \mathrm{W}$-like sequence was the dominant peptide motif binding to the $\alpha_{\mathrm{M}} \mathrm{I}$ domain.

The $\alpha_{M} \beta_{2}$ integrin is a highly promiscuos receptor binding a multitude of proteins and other biomolecules including DNA. The ability to bind multiple ligands has been attributed to the 
sequence $\mathrm{Lys}^{245}-\mathrm{Arg}^{261}$ present in the I domain (Yakubenko et al., 2002). We thus examined other known $\alpha_{M}$ integrin ligands for the occurrence of $(D / E)(D / E)(G / L) W$-like sites, which could mediate binding to the $\alpha_{M}$ I domain. The peptides containing similar sites were synthesized on a pepspot membrane and analyzed for the $\alpha_{M}$ I domain binding. Peptides from thrombospondin-1, myeloperoxidase, catalase and complement protein iC $3 \mathrm{~b}$ avidly bound the $\alpha_{M}$ I domain. If the negative charges were changed to alanines, the binding activity of these peptides was lost. These findings suggest that a similar binding mechanism is used also for other proteins than the MMPs and represent a common $\alpha_{M}$ integrin recognition motif. Supporting our results, it has been shown that elastase competes with iC3b binding to $\alpha_{M} \beta_{2}$ (Cai and Wright, 1996). Similarly, we observed competition of $\alpha_{M} I$ domain-binding $\mathrm{iC} 3 \mathrm{~b}$ peptide with the phage peptide.

The ADGACILWMDDGWCGAAG peptide (DDGW) binding to the $\alpha_{M}$ I domain was chosen as a model peptide due to its high activity and favorable solubility properties. The progelatinases bound to intact $\beta_{2}$ integrins and recombinant $\alpha_{M}$ and $\alpha_{L}$ I domains in vitro. Curiously, proMMP binding to $\alpha_{M} \beta_{2}$ integrin was about twice as strong as the binding to the $\alpha_{M}$ I domain, suggesting that other interactions outside the DDGW-like motif may augment the binding. The I domain mediated binding was inhibited by the DGGW peptide in a dose dependent manner, the $\mathrm{IC}_{50}$ value being about $25 \mu \mathrm{M}$. The interaction of proMMP-9 with the I domain was not inhibited by lovastatin, a small-molecule antagonist of $\alpha_{\mathrm{L}}$ integrin I domain. Interestingly, although the binding of proMMP-9 to the $\alpha_{M} \beta_{2}$ integrin was inhibited by the cation chelator EDTA, DDGW-peptide bearing phage binding to the I domain was cation independent (data not shown), similarly to the proMMP-1 binding to the $\alpha_{2}$ I domain (Dumin et al., 2001).

We also investigated the effect of the DDGW peptide on the adhesion and migration of THP-1 monocytic cells. Although activated THP-1 cells were able to bind to the DDGW peptide immobilized in microtiter wells, DDGW could not inhibit adhesion to the classical $\alpha_{\mathrm{M}} \beta_{2}$ ligands fibrinogen and ICAM-1. However, at a $200 \mu \mathrm{M}$ concentration DDGW peptide potently inhibited THP-1 migration on a synthetic LLG-C4-GST coating with a similar potency as the CTT peptide. DDGW had no effect on the migration of HT1080 fibrosacroma cells, which do not express $\beta_{2}$ integrins. However, HT1080 migration could be inhibited with the CTT peptide. In addition we observed that MMP-9 secretion or release from THP-1 cells is stimulated by the DDGW peptide apparently as a result of direct binding to the $\alpha_{M}$ I domain.

\section{Identification of domain-specific ligands of MMP-9 that inhibit tumor cell migration and invasion (IV)}

In order to charaterize the molecular interactions of MMP-9 in tumor cells, we performed biopanning with proMMP-9. Two new peptide motifs were isolated in the screening, one with a $\mathrm{CG}(\mathrm{Ar}) \mathrm{GR}(\mathrm{Ar})(\mathrm{S} / \mathrm{Q}) \mathrm{PPC}$ motif, where $\mathrm{Ar}$ is an aromatic amino acid, and a single peptide with CRVYGPYLLC sequence. These peptides did not compete with the CTT peptide binding and we identified that the first motif, represented by a CGYGRFSPPC (PPC) peptide, was a ligand for the collagen-binding domain (CBD) of gelatinases. The PPC peptide inhibited gelatinolysis by MMP-2 and MMP-9. The CRVYGPYLLC (CRV) peptide was found to be a ligand for the $\mathrm{C}$-terminal domain ( $\mathrm{C}$ domain). This was confirmed by phage binding to the recombinant $\mathrm{C}$ domain. Biopanning with the same domain identified similar peptides with a CRXYGPXXXC motif. The CRV peptide-bearing phage selectively 
bound proMMP-9, but not proMMP-2 and proMMP-3. However, weak but specific binding to the recombinant MMP-2 C-terminal domain was observed. Importantly, the CRV peptide did not have any effect on the gelatinolytic activity of MMP-9 in vitro.

We found a high sequence similarity between the PPC peptide and the matrix proteins fibronectin and vitronectin. The PPC-like sequence in fibronectin was located in the Cterminal heparin binding domain. The CBD of MMP-9 was found to bind to intact fibronectin but not to a $110-\mathrm{kDa}$ fragment of fibronectin lacking the $\mathrm{C}$-terminal domain. The CBD also bound to vitronectin, but less strongly, perhaps because vitronectin in our studies was in the urea-denaturated form. Interestingly, the PPC-like site in fibronectin with a TTPNSLLVSWQPPRARIT sequence is adjacent and partially overlapping the heparinbinding WQPPRARI sequence previously shown to have focal adhesion promoting activity (Woods et al., 1993). Furthermore, the WQPPRARI peptide is able to stimulate expression of MMP-1 and MMP-9 in fibroblasts plates on a fibronectin fragment lacking the heparinbinding domain. This stimulation is mediated by $\alpha_{5} \beta_{1}$ and $\alpha_{4} \beta_{1}$ integrins (Huhtala et al., 1995). We did not find evidence that PPC would act like the WQPPRARI peptide, because the active part of this peptide is the PRARI sequence (Woods et al., 1993). MMP-2 can cleave fibronectin in the heparin-binding domain. Curiously, this cleavage exposes an antiadhesive site for integrins, which suppresses cell adhesion (Watanabe et al., 2000).

We evaluated the effect of the various gelatinase-binding peptides on HT1080 fibrosarcoma cell invasion through matrigel as well as THP-1 cell migration on the LLG-C4-GST coating. All domain specific peptides, but not the scrambled control peptides inhibited migration and invasion. The activity of the CRV peptide was comparable to the CTT peptide whereas the PPC peptide required slightly higher concentrations to obtain the same efficiency. This data indicated that all three domains of the gelatinases are needed for efficient cell migration. The effect of the CTT and PPC could be explained by inhibition of gelatinase activity. Further experiments were conducted to identify the mechanism how CRV inhibits cell migration and invasion. Interestingly, although CRV could not inhibit gelatinase activity in vitro, this peptide inhibited gelatinolysis by HT1080 cells to similar extent than CTT and PPC. Despite the fact that MMP-9 is rarely seen in the active form on the cell surface, we considered the possibility that CRV could affect the activation of MMP-9. This assumption was based on the role of C-terminal domain in the activation of MMP-2. Indeed, we found that plasminogen-mediated cellular activation of MMP-9 was reduced in the presence of CRV. CRV also inhibited MMP-2 activation at higher concentrations consistent with the data that it weakly binds to the C-terminal domain of MMP-2.

\section{MMP-9 interacts with the urokinase-plasminogen activator receptor and the integrin $\beta$ chain (IV)}

The effect of the CRV peptide on plasminogen-mediated activation prompted us to examine potential interaction of MMP-9 with the urokinase-plasminogen activator receptor (UPAR). Both molecules are important players in tumor invasion. We found a physical interaction of these molecules by immunoprecipitation in HT1080 and THP-1 cells. Furthermore, MMP-9 could cleave the uPA-binding domain from uPAR in vitro. uPAR cleavage has also been observed on the cell surface, and this cleavage can be inhibited with a broad-spectrum MMP inhibitor BB-94 (Koolwijk et al., 2001). We found that a gelatinase-selective smallmolecule inhibitor and the CTT peptide inhibited the cellular cleavage of uPAR. Importantly, the cleaved form of uPAR is found in invasive transplanted tumors in mice 
(Solberg et al., 1994). Moreover, appearance of the fragmented uPAR is associated with the presence of tumor cells in acute myeloid leukemia (Mustjoki et al., 2000). These data and our findings that UPAR and MMP-9 associate in fibrosarcoma and leukemic cells indicates that the functional interplay of uPA/uPAR and MMP-9 may be a critical determinant in many types of cancer.

Given that UPAR and MMPs can interact with several integrins, we examined the possible integrin interactions of MMP-9 in HT1080 cells by immunoprecipitation. ProMMP-9 could be immunoprecipitated with antibodies to $\alpha_{5}$ and $\beta_{5}$ integrins, suggesting that the major integrin receptors in these cells are $\alpha_{5} \beta_{1}$ and $\alpha_{V} \beta_{5}$. It has been observed that the integrin associations of MMPs and UPAR are regulated by the matrix components where the cells are attached. In a serum-containing cell culture medium, the plastic becomes coated with fibronectin and vitronectin, the ligands for $\alpha_{5} \beta_{1}$ and $\alpha_{v} \beta_{5}$, respectively. It is thus not surprising to find that MMP-9 was associated with these receptors. As the C-terminal domains of MMP-1 and MMP-2 have been implicated in the integrin binding, we looked for CRV-like sequences in the integrins. A CRV-like sequence was found in the integrinepidermal growth factor-like domain 2 of the integrin $\beta$ chains. The highest similarities were found with the $\beta_{5}$ and $\beta_{6}$ integrins and a recombinant fragment of the $\beta 5$ integrin chain containing the CRV-like sequence bound to the C domain of MMP-9 and inhibited the invasion of HT1080 fibrosarcoma cells.

Despite the CRV peptide is not a direct inhibitor of gelatinases, it could potently inhibit pericellular proteolysis. To extend these findings, the effect of the CRV peptide was studied in a human tumor xenograft model. In this assay, CRV significantly inhibited human tongue squamous cell carcinoma xenograft growth prolonging the survival of the tumor-bearing mice. The scrambled control peptide was not effective. The inhibition of tumor growth could at least partly be accounted for inhibition of angiogenesis, as the CRV-treated tumors had a less developed vasculature as revealed by the endothelial marker CD31 immunostaining. In conclusion, this data suggest that compounds inhibiting MMP-9 interactions rather than direct catalytic activity may have a potential in cancer therapy.

\section{Identification of a small molecule inhibitor of $\alpha_{M} \beta_{2}$ integrin-dependent leukemia cell migration (V)}

Because peptides are not optimal therapeutic agents due to their proteolytic susceptibility and rapid clearance in vivo, we aimed at screening for small molecules that would act analogously to the peptides but be more suitable for drug-development. A competition assay with the DDGW peptide bearing phage was set up and a commercially available combinatorial library was screened. Nineteen compounds were identified as inhibitors of DDGW-peptide binding, fourteen of which had a common 2-thioxothiazolidin-4-one structure. The best compound was about six times more active than the soluble DDGW peptide in the phage assay, having an $\mathrm{IC}_{50}$ value of $0.4 \mu \mathrm{M}$. Surprisingly, these compounds could not compete with proMMP-9 binding to the $\alpha_{M}$ I domain, although they potently inhibited phage binding. In contrast, they enhanced the binding of the $\alpha_{M}$ I domain to proMMP-9 and fibrinogen suggesting that they stabilize the active conformation of the I domain.

The unexpected difference in the biological activity of DDGW and the chemical compounds can be traced to the molecular nature of these inhibitors. DDGW is a highly charged 
peptide, which is typical for the integrin ligands. Indeed, all $\alpha_{\mathrm{L}} \mathrm{I}$ domain binding small molecules described are uncharged and they bind to the same site, distinct from the ligand binding site of the I domain. This suggests that it is highly difficult to obtain direct competitors for integrin ligands and other charged peptides from combinatorial libraries. Thus, the use of phage display as a tool to screen for integrin ligands appears to provide novel ligands that cannot be easily found using combinatorial chemistry. On the contrary, phage-display peptides binding via hydrophobic interactions should readily yield direct competitors.

The most potent compound IMB-10 made $\alpha_{\mathrm{M}} \beta_{2}$ integrin-expressing cells partially resistant to detachment by the cation chelator EDTA. In addition, it was a potent inhibitor of $\beta_{2}$ integrin mediated migration of THP-1 and OCI-AML-3 leukemia cells. The IMB-10 interfered only with $\beta_{2}$ integrin-dependent migration, as there was no effect on the migration of HT1080 fibrosarcoma cells. The ability of IMB-10 to inhibit cell migration on fibrinogen was independent on gelatinase activity, as a small-molecule gelatinase inhibitor did not block cell migration in these assays. Furthermore, IMB-10 did not inhibit pericellular gelatinase-dependent proteolysis of UPAR. This data indicate that the inhibition of leukemia cell migration by IMB-10 is caused primarily due to enhanced adhesion and not by inhibition of integrin-regulated gelatinase activity.

The activation state of the I domains is regulated by the movement of the C-terminal $\alpha$ helix. In accordance with this, we found that mutations in the hydrophobic socket accommodating the $\mathrm{C}$-terminal activity-regulating helix abolished the ability of the I domain to be induced by IMB-10. In contrast, mutations in the C-terminal helix resulted in partially active I domain, which could be further induced by the chemical. We additionally confirmed that the Lys ${ }^{245}$ residue speculated to be important for progelatinase binding was indeed important. High affinity binding to proMMP-9 could not be induced with the Lys ${ }^{245}$ Ala mutant I domain.

The identified $\alpha_{M}$ I domain binding compounds, although being more potent than the DDGW peptide as an inhibitor of leukemic cell migration, are still relatively inefficient in comparison to optimized drug-molecules. It is interesting to note that from a diversity of 10 000 small-molecules, one can identify compounds that are at least as active as peptides originally derived from a library of over $10^{8}$ members. We have additionally screened the same compounds with the aid of the CRV peptide. From this screen, one potent hit was identified (M. Björklund and E. Koivunen, unpublished). The next step with the IMB-10 is to conduct structure-activity relationship studies to identify more active derivatives and to evaluate their in vivo activity. 


\section{CONCLUDING REMARKS}

Phage display is a straightforward route from basic protein-protein interaction studies to drug discovery process. Although knockout mice are considered as the golden standard when evaluating the function of different proteins in vivo, phage display peptides offer a complementary method by pharmacological inhibition of the protein function. In addition to the potential to rapidly screen for ligands for new targets and for the identification of protein-protein interactions, the peptides may be used in structural studies to identify binding sites for therapeutic small-molecules or used as a starting point in the synthesis of peptidomimetics. Novel means that would bring the peptide leads closer to the clinically useful drug molecules would significantly shorten the time and expenses needed for drug development. This work demonstrates that this goal may be attainable by using nonnatural amino acids or by selecting small-molecule compounds that mimick the action of the phage display peptides.

In this study, we used phage display of random peptides to understand gelatinase-mediated cell migration and invasion. We identified multiple interactions of gelatinases and inhibition of these interactions with the peptides was successfully used to block cancer cell migration and invasion. A favourable outcome could not only be obtained by peptides that inhibit catalytic activity of the gelatinases such as the CTT and PPC peptides, but also with the DDGW and CRV peptides, which block cell surface interactions of the gelatinases. Our results provide further evidence for the previous reports indicating a critical role of pericellular MMP activity in cell migration (Brooks et al., 1998; Brooks et al., 1996; Dumin et al., 2001). A hypothetical model of gelatinase-mediated cell migration/invasion machinery can be proposed based on these findings (Figure 9). We propose a name "invadosome" for this protein complex. In addition to the interactions characterized in our laboratory, inhibitors of interactions between uPA and UPAR as well as UPAR and integrins have been identified by phage display (Goodson et al., 1994; Wei et al., 1996). The invadosome is likely a short-lived complex where MMPs, integrins, uPA/uPAR, matrix proteins and possibly other proteins are brought together, when the cells encounter a nondegraded extracellular matrix and need to invade and migrate. Indeed, many integrin ligands are substrates for the integrin-associated proteinases. By definition, the cell migration machinery must be a highly dynamic complex to be able to perform the multiple tasks that are necessary for migration and invasion. The composition of the complex must also vary depending on the extracellular matrix ligands. Although this study does not provide direct evidence that all the components indicated in the invadosome complex would bind at the same time, the differential binding sites identified suggest that they might do so. Another point to note is that the proteinases have functions independent on their proteolytic activity. This has been well established with the uPA/uPAR system (Blasi and Carmeliet, 2002) and evidence is accumulating that the MMPs function similarily (Cao et al., 2004; Sanceau et al., 2003).

Although MMP-1, -2 and -9 bind to integrins, the interaction mechanisms appear to differ slightly. The binding of MMP-2 and MMP-9 to $\alpha_{V} \beta_{3}$ and $\alpha_{V} \beta_{5}$ appear to be the most similar. Both MMPs bind through their C-terminal domain to the integrin outside the integrin ligand-binding site (Brooks et al., 1998 and this study). However, the small-molecule inhibitor inhibiting the MMP- $2 / \alpha_{\mathrm{V}} \beta_{3}$ interaction binds to the integrin (Boger et al., 2001; Silletti et al., 2001), whereas the CRV peptide binds to the MMP-9 indicating that the exact binding mechanism need not to be the very same. The integrin $\alpha$ I domains are the binding 
sites for MMP-1 and MMP-9. Curiously, MMP-1 utilizes the C-terminal domain and the adjacent hinge region for $\alpha_{2}$ I domain binding (Stricker et al., 2001), whereas MMP-9 catalytic domain interacts with the $\alpha_{M}$ and $\alpha_{L}$ I domains. Whether this is a functional adaptation to achieve cell-type specific regulation of MMP activity remains to be determined.

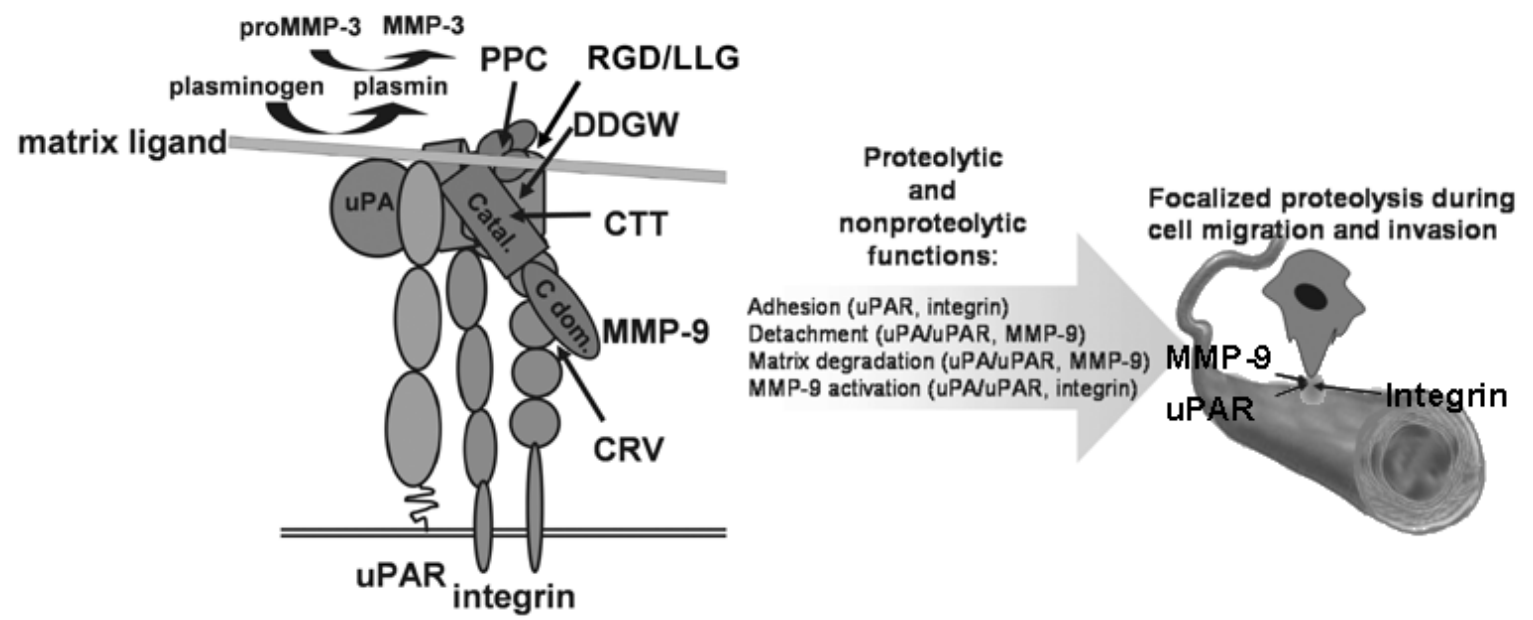

Figure 9. The invadosome model. The molecular components interacting with each other are depicted. The invadosome regulates multiple proteolytic and nonproteolytic functions, which are required for controlled cell migration and invasion for example during intravastion of the tumor cells into the blood stream.

Another issue is the relationship between the integrin activation-state and MMP binding. The $\alpha$ I domain containing integrins can exist in several conformations: 1) the inactive bent form with a closed headpiece containing the ligand binding regions; 2) extended form with closed headpiece and low affinity I domain; 3 ) extended form with open headpiece and low affinity I domain; 4) extended form with open headpiece and high affinity I domain (Shimaoka et al., 2003). The non-I domain integrins, such as $\alpha_{V} \beta_{3}$ and $\alpha_{V} \beta_{5}$ exist at least in the bent and the extendend conformation (Takagi et al., 2002; Xiong et al., 2001). The preliminary data obtained in this study indicates that the extended conformation of the integrin is required for the MMP C-terminal domain interaction. Whether MMPs can also bind to the bent form or the intermediate active forms remains to be clarified, but the ability of proMMP-9 to interact with the closed I domain suggests that this is possible. Furthermore, does activation of MMPs occur simultaneously or subsequently to integrin activation? Some studies have indicated that the activation of the proteases may occur concomitant with integrin ligand-binding and activation (Prager et al., 2003; Yan et al., 2000). Investigations with activation state-specific integrin antibodies and antibodies specific for pro- and active MMPs could shed light on this matter.

One of the major questions is whether the MMPs are a clinically relevant target for the therapeutic intervention in cancer and to what extent other diseases could be treated with the MMP inhibitors. Although over hundred small-molecules targeting the catalytic site of the MMPs have been synthesized, these compounds have limited specificity to individual MMPs and no significant success has been seen with these compounds in the clinical trials. At present, there is not sufficient knowledge on the role of individual MMPs in cancer. Hence, the decision, which MMP should be targeted still remains an educated guess. Even if such knowledge would be available, the conserved structural features of the MMPs indicate that it will be a considerable challenge to synthesize an active-site inhibitor with specificity to a single MMP. Additionally, the MMPs are typically required in the early stage of the 
tumor progression, thus the best therapeutic window for the MMP inhibitors may be lost if the disease is not early diagnosed.

It remains to be seen, whether the more selective active-site inhibitors, exosite inhibitors and inhibitors of protein-protein interactions such as those identified in this study appear to be any more successful as cancer therapeutics. Certainly a better knowledge on the role of MMPs in cancer progression is required to achieve this goal. For example, knowledge about the binding partners for the gelatinases alone is certainly incomplete. The recent finding that MMP-2 interacts with the chaperone protein Hsp90 in the extracellular space and that this interaction regulates tumor cell invasion is one indication that we do not yet understand the details about tumor cell migration and invasion (Eustace et al., 2004). Furthermore, blocking MMPs alone may not be sufficient to achieve an adequate clinical response. Hence, it is certainly worthwhile to consider the possibility of combination therapy with drugs affecting other functions of the cancer cells. Nevertheless, the peptides and chemicals identified in this study will hopefully be useful for further studies on the role of gelatinases in physiological and pathological conditions and to aid in the development of pharmacologically active agents to combat cancer and other diseases associated with excessive gelatinase activity. 


\section{ACKNOWLEDGEMENTS}

This work was carried out in the Department of Biological and Environmental Sciences (former Department of Biosciences, Division of Biochemistry) during the years 2000-2004 under the supervision of docent Erkki Koivunen.

I want to express my gratitude to the head of the Division of Biochemistry, professor Carl G. Gahmberg for providing the facilities to work in and for providing critical reagents for the work. Professors Jorma Keski-Oja and Jyrki Heino are warmly thanked for their expertise in reviewing this thesis. Kari Keinänen and Harri Savilahti are acknowledged for their supportive role as the follow-up group in the Viikki Graduate School in Biosciences. The co-authors, the past and the present lab personnel of Erkki Koivunen and all the other people in the Division of Biochemistry, and the staff in CTT Cancer Targeting Technologies Ltd. are thanked for their contribution to this work. This work was financially supported by grants from the Finnish Cultural Foundation, the Wihuri Foundation, The Ida Montin Foundation, the Finnish Academy and the Finnish Cancer Society. 


\section{REFERENCES}

Abecassis, I., Olofsson, B., Schmid, M., Zalcman, G. and Karniguian, A. (2003) RhoA induces MMP-9 expression at CD44 lamellipodial focal complexes and promotes HMEC-1 cell invasion. Exp Cell Res, 291: 363-76.

Adessi, C. and Soto, C. (2002) Converting a peptide into a drug: strategies to improve stability and bioavailability. Curr Med Chem, 9: 963-78.

Afar, D.E., Vivanco, I., Hubert, R.S., Kuo, J., Chen, E. Saffran, D.C., Raitano, A.B. and Jakobovits, A. (2001) Catalytic cleavage of the androgen-regulated TMPRSS2 protease results in its secretion by prostate and prostate cancer epithelia. Cancer Res, 61: 168692.

Agrez, M., Gu, X., Turton, J., Meldrum, C., Niu, J., Antalis, T. and Howard, E.W. (1999) The avß6 integrin induces gelatinase B secretion in colon cancer cells. Int. J. Cancer, 81: 90-7.

Aguirre Ghiso, J.A., Kovalski, K. and Ossowski, L. (1999) Tumor dormancy induced by downregulation of urokinase receptor in human carcinoma involves integrin and MAPK signaling. J Cell Biol, 147: 89-104.

Ahmed, N., Oliva, K., Wang, Y., Quinn, M. and Rice, G. (2003) Proteomic profiling of proteins associated with urokinase plasminogen activator receptor in a colon cancer cell line using an antisense approach. Proteomics, 3: 288-98.

Aimes, R.T. and Quigley, J.P. (1995) Matrix metalloproteinase- 2 is an interstitial collagenase. Inhibitor-free enzyme catalyzes the cleavage of collagen fibrils and soluble native type I collagen generating the specific 3/4- and 1/4-length fragments. $J$ Biol Chem, 270: 5872-6.

Akerman, M.E., Chan, W.C., Laakkonen, P., Bhatia, S.N. and Ruoslahti, E. (2002) Nanocrystal targeting in vivo. Proc Natl Acad Sci U S A, 99: 12617-21.

Alexander, C.M., Hansell, E.J., Behrendtsen, O., Flannery, M.L., Kishnani, N.S., Hawkes, S.P. and Werb, Z. (1996) Expression and function of matrix metalloproteinases and their inhibitors at the maternal-embryonic boundary during mouse embryo implantation. Development, 122: 1723-36.

Allan, J.A., Docherty, A.J., Barker, P.J., Huskisson, N.S., Reynolds, J.J. and Murphy, G. (1995) Binding of gelatinases $A$ and $B$ to type-I collagen and other matrix components. Biochem J, 309: 299-306.

Allan, J.A., Hembry, R.M., Angal, S., Reynolds, J.J. and Murphy, G. (1991) Binding of latent and high Mr active forms of stromelysin to collagen is mediated by the $\mathrm{C}$ terminal domain. J Cell Sci, 99: 789-95

Amour, A., Knight, C.G., Webster, A., Slocombe, P.M. Stephens, P.E., Knauper, V., Docherty, A.J. and Murphy, G. (2000) The in vitro activity of ADAM-10 is inhibited by TIMP-1 and TIMP-3. FEBS Lett, 473: 2759.

Amour, A., Slocombe, P.M., Webster, A., Butler, M., Knight, C.G., Smith, B.J., Stephens, P.E., Shelley, C., Hutton, M., Knauper, V., Docherty, A.J. and Murphy, G. (1998) TNF- $\alpha$ converting enzyme (TACE) is inhibited by TIMP3. FEBS Lett, 435: 39-44.

Andolfo, A., English, W.R., Resnati, M., Murphy, G., Blasi, F. and Sidenius, N. (2002) Metalloproteases cleave the urokinase-type plasminogen activator receptor in the D1-D2 linker region and expose epitopes not present in the intact soluble receptor. Thromb Haemost, 88: 298306.

Anthony-Cahill, S.J. and Magliery, T.J. (2002) Expanding the natural repertoire of protein structure and function. Curr Pharm Biotechnol, 3: 299-315.

Arap, W., Kolonin, M.G., Trepel, M., Lahdenranta, J., CardoVila, M., Giordano, R.J., Mintz, P.J., Ardelt, P.U., Yao, V.J., Vidal, C.I., Chen, L., Flamm, A., Valtanen, H.,
Weavind, L.M., Hicks, M.E., Pollock, R.E., Botz, G.H., Bucana, C.D., Koivunen, E., Cahill, D., Troncoso, P., Baggerly, K.A., Pentz, R.D., Do, K.A., Logothetis, C.J. and Pasqualini, R. (2002) Steps toward mapping the human vasculature by phage display. Nat Med, 8: 1217.

Arap, W., Pasqualini, R. and Ruoslahti, E. (1998) Cancer treatment by targeted drug delivery to tumor vasculature in a mouse model. Science, 279: 377-80.

Arbelaez, L.F., Bergmann, U., Tuuttila, A., Shanbhag, V.P. and Stigbrand, T. (1997) Interaction of matrix metalloproteinases-2 and -9 with pregnancy zone protein and a2-macroglobulin. Arch Biochem Biophys, 347: $62-8$.

Arnaout, M.A., Goodman, S.L. and Xiong, J.P. (2002) Coming to grips with integrin binding to ligands. Curr Opin Cell Biol, 14: 641-51.

Artym, V.V., Kindzelskii, A.L., Chen, W.T. and Petty, H.R. (2002) Molecular proximity of seprase and the urokinase-type plasminogen activator receptor on malignant melanoma cell membranes: dependence on $\beta 1$ integrins and the cytoskeleton. Carcinogenesis, 23: $1593-601$.

Ashe, B.M. and Zimmerman, M. (1977) Specific inhibition of human granulocyte elastase by cis-unsaturated fatty acids and activation by the corresponding alcohols. Biochem Biophys Res Commun, 75: 194-9.

Ashworth, J.L., Murphy, G., Rock, M.J., Sherratt, M.J. Shapiro, S.D., Shuttleworth, C.A. and Kielty, C.M. (1999) Fibrillin degradation by matrix metalloproteinases: implications for connective tissue remodelling. Biochem J, 340: 171-81.

Bacher, J.M. and Ellington, A.D. (2001) Selection and characterization of Escherichia coli variants capable of growth on an otherwise toxic tryptophan analogue. J Bacteriol, 183: 5414-25.

Backstrom, J.R. and Tokes, Z.A. (1995) The 84-kDa form of human matrix metalloproteinase- 9 degrades substance $P$ and gelatin. $J$ Neurochem, 64: 1312-8.

Bader, B. L., Rayburn, H., Crowley, D. and Hynes, R. O. (1998) Extensive vasculogenesis, angiogenesis, and organogenesis precede lethality in mice lacking all $\alpha \mathrm{v}$ integrins. Cell, 95: 507-19.

Bajorath, J. (2002) Integration of virtual and high-throughput screening. Nat Rev Drug Discov, 1: 882-94.

Balbin, M., Fueyo, A., Tester, A.M., Pendas, A.M., Pitiot, A.S., Astudillo, A., Overall, C.M., Shapiro, S.D. and Lopez-Otin, C. (2003) Loss of collagenase-2 confers increased skin tumor susceptibility to male mice. Nat Genet, 35: 252-7.

Baluk, P., Raymond, W.W., Ator, E., Coussens, L.M., McDonald, D.M. and Caughey, G.H. (2004) Matrix metalloproteinase 2 and 9 expression increases in mycoplasma-infected airways but is not required for microvascular remodeling. Am J Physiol Lung Cell Mol Physiol, 287: L307-17.

Bannikov, G.A., Karelina, T.V., Collier, I.E., Marmer, B.L. and Goldberg, G.I. (2002) Substrate binding of gelatinase $B$ induces its enzymatic activity in the presence of intact propeptide. J Biol Chem, 277: 16022-7.

Bansal, V. S., Vaidya, S., Somers, E. P., Kanuga, M., Shevell, D., Weikel, R. and Detmers, P. A. (2003) Small molecule antagonists of complement receptor type 3 block adhesion and adhesion-dependent oxidative burst in human polymorphonuclear leukocytes. $J$ Pharmacol Exp Ther, 304: 1016-24.

Banyard, J., Anand-Apte, B., Symons, M. and Zetter, B.R. (2000) Motility and invasion are differentially modulated by Rho family GTPases. Oncogene, 19: 580-91. 
Barnhill, R.L., Piepkorn, M.W., Cochran, A.J., Flynn, E., Karaoli, T. and Folkman, J. (1998) Tumor vascularity, proliferation, and apoptosis in human melanoma micrometastases and macrometastases. Arch Dermatol, 134: 991-4.

Bax, D.V., Messent, A.J., Tart, J., Van Hoang, M., Kott, J., Maciewicz, R.A. and Humphries, M.J. (2004) Integrin $\alpha 5 \beta 1$ and ADAM-17 Interact in Vitro and Co-localize in Migrating HeLa Cells. J Biol Chem, 279: 22377-22386.

Behrendtsen, O., Alexander, C.M. and Werb, Z. (1992) Metalloproteinases mediate extracellular matrix degradation by cells from mouse blastocyst outgrowths. Development, 114: 447-56.

Bein, K. and Simons, M. (2000) Thrombospondin type 1 repeats interact with matrix metalloproteinase 2 . Regulation of metalloproteinase activity. J Biol Chem, 275: 32167-73.

Bello, L., Lucini, V., Carrabba, G., Giussani, C., Machluf, M., Pluderi, M., Nikas, D., Zhang, J., Tomei, G., Villani, R.M., Carroll, R.S., Bikfalvi, A. and Black, P.M. (2001) Simultaneous inhibition of glioma angiogenesis, cell proliferation, and invasion by a naturally occurring fragment of human metalloproteinase-2. Cancer Res, 61: 8730-6.

Bergers, G., Brekken, R., McMahon, G., Vu, T.H., Itoh, T., Tamaki, K., Tanzawa, K., Thorpe, P., Itohara, S., Werb, Z. and Hanahan, D. (2000) Matrix metalloproteinase-9 triggers the angiogenic switch during carcinogenesis. Nat Cell Biol, 2: 737-44.

Bergers, G., Javaherian, K., Lo, K.M., Folkman, J. and Hanahan, D. (1999) Effects of angiogenesis inhibitors on multistage carcinogenesis in mice. Science, 284 808-12.

Bernardo, M.M., Brown, S., Li, Z.H., Fridman, R. and Mobashery, S. (2002) Design, synthesis, and characterization of potent, slow-binding inhibitors that are selective for gelatinases. J Biol Chem, 277: 112017. Epub 2002 Jan 14.

Berton, A., Godeau, G., Emonard, H., Baba, K., Bellon, P., Hornebeck, W. and Bellon, G. (2000) Analysis of the ex vivo specificity of human gelatinases $A$ and $B$ towards skin collagen and elastic fibers by computerized morphometry. Matrix Biol, 19: 139-48.

Berton, A., Rigot, V., Huet, E., Decarme, M., Eeckhout, Y. Patthy, L., Godeau, G., Hornebeck, W., Bellon, G. and Emonard, H. (2001) Involvement of fibronectin type II repeats in the efficient inhibition of gelatinases $A$ and $B$ by long-chain unsaturated fatty acids. $\mathrm{J} \mathrm{Biol} \mathrm{Chem,}$ 276: 20458-65.

Betsuyaku, T., Shipley, J.M., Liu, Z. and Senior, R.M. (1999) Neutrophil emigration in the lungs, peritoneum, and skin does not require gelatinase B. Am J Respir Cell Mol Biol, 20: 1303-9.

Bini, A., Itoh, Y., Kudryk, B.J. and Nagase, H. (1996) Degradation of cross-linked fibrin by matrix metalloproteinase 3 (stromelysin 1): hydrolysis of the gamma Gly 404-Ala 405 peptide bond. Biochemistry 35: 13056-63.

Birkedal-Hansen, H., Moore, W.G., Bodden, M.K., Windsor, L.J., Birkedal-Hansen, B., DeCarlo, A. and Engler, J.A. (1993) Matrix metalloproteinases: a review. Crit Rev Oral Biol Med, 4: 197-250.

Black, R. A. (2002) Tumor necrosis factor- $\alpha$ converting enzyme. Int J Biochem Cell Biol, 34: 1-5.

Blasi, F. and Carmeliet, P. (2002) UPAR: a versatile signalling orchestrator. Nat Rev Mol Cell Biol, 3: 93243.

Boger, D.L., Goldberg, J., Silletti, S., Kessler, T. and Cheresh, D.A. (2001) Identification of a novel class of small-molecule antiangiogenic agents through the screening of combinatorial libraries which function by inhibiting the binding and localization of proteinase MMP2 to integrin avß3. J Am Chem Soc, 123: 1280-8.

Borregaard, N. (1997) Development of neutrophil granule diversity. Ann N Y Acad Sci, 832: 62-8.
Bourguignon, L.Y., Gunja-Smith, Z., lida, N., Zhu, H.B., Young, L.J., Muller, W.J. and Cardiff, R.D. (1998) CD44v $(3,8-10)$ is involved in cytoskeleton-mediated tumor cell migration and matrix metalloproteinase (MMP-9) association in metastatic breast cancer cells. J Cell Physiol, 176: 206-15.

Bramhall, S.R., Hallissey, M.T., Whiting, J., Scholefield, J., Tierney, G., Stuart, R.C., Hawkins, R.E., McCulloch, P., Maughan, T., Brown, P.D., Baillet, M. and Fielding, J.W. (2002) Marimastat as maintenance therapy for patients with advanced gastric cancer: a randomised trial. Br J Cancer, 86: 1864-70.

Bramhall, S.R., Schulz, J., Nemunaitis, J., Brown, P.D., Baillet, M. and Buckels, J.A. (2002) A double-blind placebo-controlled, randomised study comparing gemcitabine and marimastat with gemcitabine and placebo as first line therapy in patients with advanced pancreatic cancer. Br J Cancer, 87: 161-7.

Bremer, C., Tung, C.H. and Weissleder, R. (2001) In vivo molecular target assessment of matrix metalloproteinase inhibition. Nat Med, 7: 743-8.

Bridges, L.C., Tani, P.H., Hanson, K.R., Roberts, C.M., Judkins, M.B. and Bowditch, R.D. (2002) The lymphocyte metalloprotease MDC-L (ADAM 28) is a ligand for the integrin a4ß1. J Biol Chem, 277: 3784-92.

Briknarova, K., Gehrmann, M., Banyai, L., Tordai, H., Patthy, L. and Llinas, M. (2001) Gelatin-binding region of human matrix metalloproteinase-2: solution structure, dynamics, and function of the COL-23 two-domain construct. J Biol Chem, 276: 27613-21.

Briknarova, K., Grishaev, A., Banyai, L., Tordai, H., Patthy, L. and Llinas, M. (1999) The second type II module from human matrix metalloproteinase 2: structure, function and dynamics. Structure Fold Des, 7: 1235-45.

Brooks, P.C., Klemke, R.L., Schon, S., Lewis, J.M., Schwartz, M.A. and Cheresh, D.A. (1997) Insulin-like growth factor receptor cooperates with integrin $\alpha v \beta 5$ to promote tumor cell dissemination in vivo. J Clin Invest, 99: $1390-8$.

Brooks, P.C., Montgomery, A.M., Rosenfeld, M., Reisfeld, R.A., Hu, T., Klier, G. and Cheresh, D.A. (1994) Integrin $\alpha v \beta 3$ antagonists promote tumor regression by inducing apoptosis of angiogenic blood vessels. Cell, 79: $1157-64$

Brooks, P.C., Silletti, S., von Schalscha, T.L., Friedlander, M. and Cheresh, D.A. (1998) Disruption of angiogenesis by PEX, a noncatalytic metalloproteinase fragment with integrin binding activity. Cell, 92: 391 400.

Brooks, P.C., Stromblad, S., Sanders, L.C., von Schalscha, T.L., Aimes, R.T., Stetler-Stevenson, W.G., Quigley, J.P. and Cheresh, D.A. (1996) Localization of matrix metalloproteinase MMP-2 to the surface of invasive cells by interaction with integrin av $\beta 3$. Cell, 85: 683-93.

Brown, P.D. (2000) Ongoing trials with matrix metalloproteinase inhibitors. Expert Opin Investig Drugs, 9: 2167-77.

Budisa, N., Steipe, B., Demange, P., Eckerskorn, C. Kellermann, J. and Huber, R. (1995) High-level biosynthetic substitution of methionine in proteins by its analogs 2-aminohexanoic acid, selenomethionine, telluromethionine and ethionine in Escherichia coli. Eur J Biochem, 230: 788-96.

Butler, G.S., Tam, E.M. and Overall, C.M. (2004) The Canonical Methionine 392 of Matrix Metalloproteinase 2 (Gelatinase A) Is Not Required for Catalytic Efficiency or Structural Integrity: Probing the Role of the Methionine-turn in the Metzincin metalloproteinase superfamily. J Biol Chem, 279: 15615-20.

Cai, T.Q. and Wright, S.D. (1996) Human leukocyte elastase is an endogenous ligand for the integrin CR3 (CD11b/CD18, Mac-1, $\alpha M \beta 2$ ) and modulates polymorphonuclear leukocyte adhesion. J Exp Med, 184: 1213-23. 
Cane, D.E., Walsh, C.T. and Khosla, C. (1998) Harnessing the biosynthetic code: combinations, permutations, and mutations. Science, 282: 63-8.

Cao, J., Kozarekar, P., Pavlaki, M., Chiarelli, C., Bahou, W.F. and Zucker, S. (2004) Distinct roles for the catalytic and hemopexin domains of membrane type 1matrix metalloproteinase in substrate degradation and cell migration. J Biol Chem, 279: 14129-39.

Carmeliet, P., Moons, L., Lijnen, R., Baes, M., Lemaitre, V., Tipping, P., Drew, A., Eeckhout, Y., Shapiro, S., Lupu, F. and Collen, D. (1997) Urokinase-generated plasmin activates matrix metalloproteinases during aneurysm formation. Nat Genet, 17: 439-44.

Carriero, M.V., Del Vecchio, S., Capozzoli, M., Franco, P. Fontana, L., Zannetti, A., Botti, G., D'Aiuto, G., Salvatore, M. and Stoppelli, M.P. (1999) Urokinase receptor interacts with av $\beta 5$ vitronectin receptor, promoting urokinase-dependent cell migration in breast cancer. Cancer Res, 59: 5307-14.

Caudroy, S., Polette, M., Nawrocki-Raby, B., Cao, J., Toole, B.P., Zucker, S. and Birembaut, P. (2002) EMMPRINmediated MMP regulation in tumor and endothelial cells. Clin Exp Metastasis, 19: 697-702.

Cha, H., Kopetzki, E., Huber, R., Lanzendorfer, M. and Brandstetter, H. (2002) Structural basis of the adaptive molecular recognition by MMP9. J Mol Biol, 320: 106579 .

Chandler, S., Coates, R., Gearing, A., Lury, J., Wells, G. and Bone, E. (1995) Matrix metalloproteinases degrade myelin basic protein. Neurosci Lett, 201: 223-6.

Chapman, H.A. (1997) Plasminogen activators, integrins, and the coordinated regulation of cell adhesion and migration. Curr Opin Cell Biol, 9: 714-24.

Chen, E.I., Kridel, S.J., Howard, E.W., Li, W., Godzik, A. and Smith, J.W. (2002) A unique substrate recognition profile for matrix metalloproteinase-2. J Biol Chem, 277: 4485-91.

Chen, E.I., Li, W., Godzik, A., Howard, E.W. and Smith, J.W. (2003) A residue in the S2 subsite controls substrate selectivity of matrix metalloproteinase-2 and matrix metalloproteinase-9. J Biol Chem, 278: 17158-63.

Chen, W.T. (1981) Mechanism of retraction of the trailing edge during fibroblast movement. J Cell Biol, 90: 187200.

Chen, W.T. (1996) Proteases associated with invadopodia, and their role in degradation of extracellular matrix. Enzyme Protein, 49: 59-71.

Chen, W.T. and Wang, J.Y. (1999) Specialized surface protrusions of invasive cells, invadopodia and lamellipodia, have differential MT1-MMP, MMP-2, and TIMP-2 localization. Ann N Y Acad Sci, 878: 361-71.

Cheng, S. and Lovett, D.H. (2003) Gelatinase A (MMP-2) is necessary and sufficient for renal tubular cell epithelialmesenchymal transformation. Am J Pathol, 162: 193749.

Chin, J.W., Cropp, T.A., Anderson, J.C., Mukherji, M., Zhang, Z. and Schultz, P.G. (2003) An expanded eukaryotic genetic code. Science, 301: 964-7.

Chin, J.W., Martin, A.B., King, D.S., Wang, L. and Schultz, P.G. (2002) Addition of a photocrosslinking amino acid to the genetic code of Escherichiacoli. Proc Natl Acad Sci U S A, 99: 11020-4.

Chong, S., Mersha, F.B., Comb, D.G., Scott, M.E., Landry, D., Vence, L.M., Perler, F.B., Benner, J., Kucera, R.B., Hirvonen, C.A., Pelletier, J.J., Paulus, H. and Xu, M.Q. (1997) Single-column purification of free recombinant proteins using a self-cleavable affinity tag derived from a protein splicing element. Gene, 192: 271-81.

Chong, S., Montello, G.E., Zhang, A., Cantor, E.J., Liao, W. Xu, M.Q. and Benner, J. (1998) Utilizing the C-terminal cleavage activity of a protein splicing element to purify recombinant proteins in a single chromatographic step. Nucleic Acids Res, 26: 5109-15.

Cianfrocca, M., Cooley, T.P., Lee, J.Y., Rudek, M.A. Scadden, D.T., Ratner, L., Pluda, J.M., Figg, W.D., Krown, S.E. and Dezube, B.J. (2002) Matrix metalloproteinase inhibitor COL-3 in the treatment of AIDS-related Kaposi's sarcoma: a phase I AIDS malignancy consortium study. J Clin Oncol, 20: 153-9.

Clark, E.A., Golub, T.R., Lander, E.S. and Hynes, R.O. (2000) Genomic analysis of metastasis reveals an essential role for RhoC. Nature, 406: 532-5.

Cole, A.A., Boyd, T., Luchene, L., Kuettner, K.E. and Schmid, T.M. (1993) Type X collagen degradation in long-term serum-free culture of the embryonic chick tibia following production of active collagenase and gelatinase. Dev Biol, 159: 528-34.

Collier, I.E., Krasnov, P.A., Strongin, A.Y., Birkedal-Hansen, H. and Goldberg, G.I. (1992) Alanine scanning mutagenesis and functional analysis of the fibronectinlike collagen-binding domain from human 92-kDa type IV collagenase. J Biol Chem, 267: 6776-81.

Collier, I.E., Saffarian, S., Marmer, B.L., Elson, E.L. and Goldberg, G. (2001) Substrate recognition by gelatinase $\mathrm{A}$ : the $\mathrm{C}$-terminal domain facilitates surface diffusion. Biophys J, 81: 2370-7.

Cornish, V.W., Benson, D.R., Altenbach, C.A., Hideg, K., Hubbell, W.L. and Schultz, P.G. (1994) Site-specific incorporation of biophysical probes into proteins. Proc Natl Acad Sci U S A, 91: 2910-15.

Corry, D.B., Kiss, A., Song, L.Z., Song, L., Xu, J., Lee, S.H., Werb, Z. and Kheradmand, F. (2004) Overlapping and independent contributions of MMP2 and MMP9 to lung allergic inflammatory cell egression through decreased CC chemokines. FASEB J, 18: 995-7.

Corry, D.B., Rishi, K., Kanellis, J., Kiss, A., Song Lz, L.Z., Xu, J., Feng, L., Werb, Z. and Kheradmand, F. (2002) Decreased allergic lung inflammatory cell egression and increased susceptibility to asphyxiation in MMP2deficiency. Nat Immunol, 3: 347-53.

Cottingham, I.R., Millar, A., Emslie, E., Colman, A. Schnieke, A.E. and McKee, C. (2001) A method for the amidation of recombinant peptides expressed as intein fusion proteins in Escherichia coli. Nat Biotechnol, 19: 974-7.

Coussens, L.M., Fingleton, B. and Matrisian, L.M. (2002) Matrix metalloproteinase inhibitors and cancer: trials and tribulations. Science, 295: 2387-92.

Coussens, L.M., Raymond, W.W., Bergers, G., LaigWebster, M., Behrendtsen, O., Werb, Z., Caughey, G.H. and Hanahan, D. (1999) Inflammatory mast cells up-regulate angiogenesis during squamous epithelial carcinogenesis. Genes Dev, 13: 1382-97.

Coussens, L.M., Tinkle, C.L., Hanahan, D. and Werb, Z. (2000) MMP-9 supplied by bone marrow-derived cells contributes to skin carcinogenesis. Cell, 103: 481-90.

Coussens, L.M. and Werb, Z. (2002) Inflammation and cancer. Nature, 420: 860-7.

Cowie, D.B. and Cohen, G.N. (1957) Biosynthesis by Escherichia coli of active altered proteins containing selenium instead of sulfur. Biochim Biophys Acta, 26: 252-61.

Cowland, J.B. and Borregaard, N. (1999) The individual regulation of granule protein mRNA levels during neutrophil maturation explains the heterogeneity of neutrophil granules. J Leukoc Biol, 66: 989-95.

Crabbe, T., Ioannou, C. and Docherty, A.J. (1993) Human progelatinase $A$ can be activated by autolysis at a rate that is concentration-dependent and enhanced by heparin bound to the C-terminal domain. Eur $J$ Biochem, 218: 431-8.

Crabbe, T., O'Connell, J.P., Smith, B.J. and Docherty, A.J. (1994) Reciprocated matrix metalloproteinase activation: a process performed by interstitial collagenase and progelatinase A. Biochemistry, 33 14419-25.

Cristofanilli, M., Charnsangavej, C. and Hortobagyi, G.N. (2002) Angiogenesis modulation in cancer research: novel clinical approaches. Nat Rev Drug Discov, 1: 415-26.

Curnis, F., Sacchi, A., Borgna, L., Magni, F., Gasparri, A. and Corti, A. (2000) Enhancement of tumor necrosis 
factor- $\alpha$ antitumor immunotherapeutic properties by targeted delivery to aminopeptidase N (CD13). Nat Biotechnol, 18: 1185-90.

Czekay, R.P., Aertgeerts, K., Curriden, S.A. and Loskutoff, D.J. (2003) Plasminogen activator inhibitor-1 detaches cells from extracellular matrices by inactivating integrins. J Cell Biol, 160: 781-91.

Dano, K., Andreasen, P. A., Grondahl-Hansen, J., Kristensen, P., Nielsen, L. S. and Skriver, L. (1985) Plasminogen activators, tissue degradation, and cancer. Adv Cancer Res, 44: 139-266

Davies, B., Brown, P.D., East, N., Crimmin, M.J. and Balkwill, F.R. (1993) A synthetic matrix metalloproteinase inhibitor decreases tumor burden and prolongs survival of mice bearing human ovarian carcinoma xenografts. Cancer Res, 53: 2087-91.

De, S., Chen, J., Narizhneva, N.V., Heston, W., Brainard, J., Sage, E.H. and Byzova, T.V. (2003) Molecular pathway for cancer metastasis to bone. J Biol Chem, 278: 39044-50.

Dedkova, L.M., Fahmi, N.E., Golovine, S.Y. and Hecht, S.M. (2003) Enhanced D-amino acid incorporation into protein by modified ribosomes. J Am Chem Soc, 125 : 6616-7.

Demeule, M., Brossard, M., Page, M., Gingras, D. and Beliveau, R. (2000) Matrix metalloproteinase inhibition by green tea catechins. Biochim Biophys Acta, 1478: 51-60.

Deng, S.J., Bickett, D.M., Mitchell, J.L., Lambert, M.H., Blackburn, R.K., Carter, H.L., 3rd, Neugebauer, J., Pahel, G., Weiner, M.P. and Moss, M.L. (2000) Substrate specificity of human collagenase 3 assessed using a phage-displayed peptide library. $\mathrm{J} \mathrm{Bio} / \mathrm{Chem}$ 275: 31422-7.

Derbyshire, V., Wood, D.W., Wu, W., Dansereau, J.T., Dalgaard, J.Z. and Belfort, M. (1997) Genetic definition of a protein-splicing domain: functional mini-inteins support structure predictions and a model for intein evolution. Proc Natl Acad Sci U S A, 94: 11466-71.

Deryugina, E.I., Ratnikov, B., Monosov, E., Postnova, T.I., DiScipio, R., Smith, J.W. and Strongin, A.Y. (2001) MT1-MMP initiates activation of pro-MMP-2 and integrin $\alpha \mathrm{v} \beta 3$ promotes maturation of MMP-2 in breast carcinoma cells. Exp Cell Res, 263: 209-23.

Descamps, F.J., Van den Steen, P.E., Martens, E., Ballaux, F., Geboes, K. and Opdenakker, G. (2003) Gelatinase $B$ is diabetogenic in acute and chronic pancreatitis by cleaving insulin. FASEB J, 17: 887-9.

Deshayes, K., Schaffer, M.L., Skelton, N.J., Nakamura, G.R., Kadkhodayan, S. and Sidhu, S.S. (2002) Rapid identification of small binding motifs with highthroughput phage display: discovery of peptidic antagonists of IGF-1 function. Chem Biol, 9: 495-505.

Dettman, H.D., Weiner, J.H. and Sykes, B.D. (1982) 19F nuclear magnetic resonance studies of the coat protein of bacteriophage M13 in synthetic phospholipid vesicles and deoxycholate micelles. Biophys J, 37: 243-51.

D'Haese, A., Wuyts, A., Dillen, C., Dubois, B., Billiau, A., Heremans, H., Van Damme, J., Arnold, B. and Opdenakker, G. (2000) In vivo neutrophil recruitment by granulocyte chemotactic protein- 2 is assisted by gelatinase B/MMP-9 in the mouse. J Interferon Cytokine Res, 20: 667-74.

Dhanasekaran, S.M., Barrette, T.R., Ghosh, D., Shah, R., Varambally, S., Kurachi, K., Pienta, K.J., Rubin, M.A. and Chinnaiyan, A.M. (2001) Delineation of prognostic biomarkers in prostate cancer. Nature, 412: 822-6.

DiPersio, C.M., Shao, M., Di Costanzo, L., Kreidberg, J.A. and Hynes, R.O. (2000) Mouse keratinocytes immortalized with large $T$ antigen acquire $\alpha 3 \beta 1$ integrindependent secretion of MMP-9/gelatinase B. J Cell Sci, 113: $2909-21$

Dolo, V., D'Ascenzo, S., Violini, S., Pompucci, L., Festuccia, C., Ginestra, A., Vittorelli, M.L., Canevari, S. and Pavan, A. (1999) Matrix-degrading proteinases are shed in membrane vesicles by ovarian cancer cells in vivo and in vitro. Clin Exp Metastasis, 17: 131-40.

Dolo, V., Li, R., Dillinger, M., Flati, S., Manela, J., Taylor, B.J., Pavan, A. and Ladisch, S. (2000) Enrichment and localization of ganglioside G(D3) and caveolin-1 in shed tumor cell membrane vesicles. Biochim Biophys Acta, 1486: 265-74.

Doring, V., Mootz, H.D., Nangle, L.A., Hendrickson, T.L., de Crecy-Lagard, V., Schimmel, P. and Marliere, P. (2001) Enlarging the amino acid set of Escherichia coli by infiltration of the valine coding pathway. Science, 292 501-4.

Dubois, B., Arnold, B. and Opdenakker, G. (2000) Gelatinase B deficiency impairs reproduction. J Clin Invest, 106: 627-8.

Dubois, B., Masure, S., Hurtenbach, U., Paemen, L., Heremans, H., van den Oord, J., Sciot, R., Meinhardt, T., Hammerling, G., Opdenakker, G. and Arnold, B. (1999) Resistance of young gelatinase B-deficient mice to experimental autoimmune encephalomyelitis and necrotizing tail lesions. J Clin Invest, 104: 1507-15.

Dumin, J.A., Dickeson, S.K., Stricker, T.P., BhattacharyyaPakrasi, M., Roby, J.D., Santoro, S.A. and Parks, W.C. (2001) Pro-collagenase-1 (matrix metalloproteinase-1) binds the $\alpha 2 \beta 1$ integrin upon release from keratinocytes migrating on type I collagen. J Biol Chem, 276: 2936874.

Dunn, S.E., Torres, J.V., Oh, J.S., Cykert, D.M. and Barrett, J.C. (2001) Up-regulation of urokinase-type plasminogen activator by insulin-like growth factor-I depends upon phosphatidylinositol-3 kinase and mitogen-activated protein kinase kinase. Cancer Res, 61: 1367-74.

Dupont, E., Savard, P.E., Jourdain, C., Juneau, C., Thibodeau, A., Ross, N., Marenus, K., Maes, D.H., Pelletier, G. and Sauder, D.N. (1998) Antiangiogenic properties of a novel shark cartilage extract: potential role in the treatment of psoriasis. J Cutan Med Surg, 2: 146-52.

Dwyer, M.A., Lu, W., Dwyer, J.J. and Kossiakoff, A.A. (2000) Biosynthetic phage display: a novel protein engineering tool combining chemical and genetic diversity. Chem Biol, 7: 263-74.

Egeblad, M. and Werb, Z. (2002) New functions for the matrix metalloproteinases in cancer progression. Nat Rev Cancer, 2: 161-74.

Eliceiri, B.P., Puente, X.S., Hood, J.D., Stupack, D.G., Schlaepfer, D.D., Huang, X.Z., Sheppard, D. and Cheresh, D.A. (2002) Src-mediated coupling of focal adhesion kinase to integrin $\alpha v \beta 5$ in vascular endothelial growth factor signaling. J Cell Biol, 157: 149-60.

Elkins, P.A., Ho, Y.S., Smith, W.W., Janson, C.A., D'Alessio K.J., McQueney, M.S., Cummings, M.D. and Romanic, A.M. (2002) Structure of the C-terminally truncated human ProMMP9, a gelatin-binding matrix metalloproteinase. Acta Crystallogr D Biol Crystallogr, 58: 1182-92

Ellerbroek, S.M., Halbleib, J.M., Benavidez, M., Warmka, J.K., Wattenberg, E.V., Stack, M.S. and Hudson, L.G. (2001) Phosphatidylinositol 3-kinase activity in epidermal growth factor-stimulated matrix metalloproteinase- 9 production and cell surface association. Cancer Res, 61: 1855-61.

Ellis, V. (2003) Plasminogen activation at the cell surface. In Zucker, S. and Chen, W. (eds.), Cell surface proteases. Academic Press, New York, Vol. 54, pp. 263-312.

Ellis, V., Whawell, S.A., Werner, F. and Deadman, J.J. (1999) Assembly of urokinase receptor-mediated plasminogen activation complexes involves direct, nonactive-site interactions between urokinase and plasminogen. Biochemistry, 38: 651-9.

Engels, M.F. and Venkatarangan, P. (2001) Smart screening: approaches to efficient HTS. Curr Opin Drug Discov Devel, 4: 275-83.

Eustace, B.K., Sakurai, T., Stewart, J.K., Yimlamai, D., Unger, C., Zehetmeier, C., Lain, B., Torella, C., 
Henning, S.W., Beste, G., Scroggins, B.T., Neckers, L., Ilag, L.L. and Jay, D.G. (2004) Functional proteomic screens reveal an essential extracellular role for hsp90 $\alpha$ in cancer cell invasiveness. Nat Cell Biol, 6: 507-14.

Evans, T.C., Jr., Benner, J. and Xu, M.Q. (1999) The cyclization and polymerization of bacterially expressed proteins using modified self-splicing inteins. $J$ Biol Chem, 274: 18359-63.

Falardeau, P., Champagne, P., Poyet, P., Hariton, C. and Dupont, E. (2001) Neovastat, a naturally occurring multifunctional antiangiogenic drug, in phase III clinical trials. Semin Oncol, 28: 620-5.

Fang, K.C., Raymond, W.W., Blount, J.L. and Caughey, G.H. (1997) Dog mast cell $\alpha$-chymase activates progelatinase $B$ by cleaving the Phe88-GIn89 and Phe91-Glu92 bonds of the catalytic domain. J Biol Chem, 272: 25628-35.

Fazioli, F., Resnati, M., Sidenius, N., Higashimoto, Y., Appella, E. and Blasi, F. (1997) A urokinase-sensitive region of the human urokinase receptor is responsible for its chemotactic activity. EMBO J, 16: 7279-86.

Fedarko, N.S., Jain, A., Karadag, A. and Fisher, L.W. (2004) Three small integrin binding ligand $\mathrm{N}$-linked glycoproteins (SIBLINGs) bind and activate specific matrix metalloproteinases. FASEB J, 18: 734-6.

Felbor, U., Dreier, L., Bryant, R.A., Ploegh, H.L., Olsen, B.R. and Mothes, W. (2000) Secreted cathepsin L generates endostatin from collagen XVIII. EMBO J, 19: 1187-94.

Felici, F., Castagnoli, L., Musacchio, A., Jappelli, R. and Cesareni, G. (1991) Selection of antibody ligands from a large library of oligopeptides expressed on a multivalent exposition vector. J Mol Biol, 222: 301-10.

Fenster, E.D. and Anker, H.S. (1969) Incorporation into polypeptide and charging on transfer ribonucleic acid of the amino acid analog $5^{\prime}, 5^{\prime}, 5^{\prime}$-trifluoroleucine by leucine auxotrophs of Escherichia coli. Biochemistry, 8: 269-74.

Fernandez-Patron, C., Martinez-Cuesta, M.A., Salas, E., Sawicki, G., Wozniak, M., Radomski, M.W. and Davidge, S.T. (1999) Differential regulation of platelet aggregation by matrix metalloproteinases- 9 and -2 . Thromb Haemost, 82: 1730-5.

Fernandez-Patron, C., Stewart, K.G., Zhang, Y., Koivunen, E., Radomski, M.W. and Davidge, S.T. (2000) Vascular matrix metalloproteinase-2-dependent cleavage of calcitonin gene-related peptide promotes vasoconstriction. Circ Res, 87: 670-6.

Fernandez-Patron, C., Zouki, C., Whittal, R.M., Chan, J.S., Davidge, S.T. and Filep, J.J. (2002) Methods for Analysis of Matrix Metalloproteinase Regulation of Neutrophil-Endothelial Cell Adhesion. Biol Proced Online, 4: 38-48.

Ferreras, M., Felbor, U., Lenhard, T., Olsen, B.R. and Delaisse, J. (2000) Generation and degradation of human endostatin proteins by various proteinases. FEBS Lett, 486: 247-51.

Festuccia, C., Angelucci, A., Gravina, G.L., Villanova, I., Teti, A., Albini, A., Bologna, M. and Abini, A. (2000) Osteoblast-derived TGF- $\beta 1$ modulates matrix degrading protease expression and activity in prostate cancer cells. Int J Cancer, 85: 407-15.

Filardo, E.J., Brooks, P.C., Deming, S.L., Damsky, C. and Cheresh, D.A. (1995) Requirement of the NPXY motif in the integrin $\beta 3$ subunit cytoplasmic tail for melanoma cell migration in vitro and in vivo. $J$ Cell Biol, 130: 44150.

Fiore, E., Fusco, C., Romero, P. and Stamenkovic, I. (2002) Matrix metalloproteinase 9 (MMP-9/gelatinase B) proteolytically cleaves ICAM-1 and participates in tumor cell resistance to natural killer cell-mediated cytotoxicity. Oncogene, 21: 5213-23.

Folkman, J. (1992) The role of angiogenesis in tumor growth. Semin Cancer Biol, 3: 65-71.

Fong, K.M., Kida, Y., Zimmerman, P.V. and Smith, P.J. (1996) TIMP1 and adverse prognosis in non-small cell lung cancer. Clin Cancer Res, 2: 1369-72.
Fosang, A.J., Neame, P.J., Last, K., Hardingham, T.E., Murphy, G. and Hamilton, J.A. (1992) The interglobular domain of cartilage aggrecan is cleaved by PUMP, gelatinases, and cathepsin B. J Biol Chem, 267: 194704.

Fotouhi, N., Lugo, A., Visnick, M., Lusch, L., Walsky, R., Coffey, J.W. and Hanglow, A.C. (1994) Potent peptide inhibitors of stromelysin based on the prodomain region of matrix metalloproteinases. J Biol Chem, 269: 3022731.

Fowlkes, J.L., Enghild, J.J., Suzuki, K. and Nagase, H. (1994) Matrix metalloproteinases degrade insulin-like growth factor-binding protein-3 in dermal fibroblast cultures. J Biol Chem, 269: 25742-6.

Frankel, A., Li, S., Starck, S.R. and Roberts, R.W. (2003) Unnatural RNA display libraries. Curr Opin Struct Biol, 13: $506-12$.

Franzke, C.W., Tasanen, K., Schacke, H., Zhou, Z., Tryggvason, K., Mauch, C., Zigrino, P., Sunnarborg, S., Lee, D.C., Fahrenholz, F. and Bruckner-Tuderman, L. (2002) Transmembrane collagen XVII, an epithelial adhesion protein, is shed from the cell surface by ADAMs. EMBO J, 21: 5026-35.

Fridman, R., Toth, M., Pena, D. and Mobashery, S. (1995) Activation of progelatinase B (MMP-9) by gelatinase A (MMP-2). Cancer Res, 55: 2548-55.

Friedl, P. and Brocker, E.B. (2000) The biology of cell locomotion within three-dimensional extracellular matrix. Cell Mol Life Sci, 57: 41-64.

Friedlander, M., Brooks, P.C., Shaffer, R.W., Kincaid, C.M., Varner, J.A. and Cheresh, D.A. (1995) Definition of two angiogenic pathways by distinct $\alpha v$ integrins. Science, 270: $1500-2$.

Fuh, G., Pisabarro, M.T., Li, Y., Quan, C., Lasky, L.A. and Sidhu, S.S. (2000) Analysis of PDZ domain-ligand interactions using carboxyl-terminal phage display. $J$ Biol Chem, 275: 21486-91.

Fuh, G. and Sidhu, S.S. (2000) Efficient phage display of polypeptides fused to the carboxy-terminus of the M13 gene-3 minor coat protein. FEBS Lett, 480: 231-4.

Furter, R. (1998) Expansion of the genetic code: sitedirected p-fluoro-phenylalanine incorporation in Escherichia coli. Protein Sci, 7: 419-26.

Gahmberg, C. G. (1997) Leukocyte adhesion: CD11/CD18 integrins and intercellular adhesion molecules. Curr Opin Cell Biol, 9: 643-50.

Galvez, B.G., Matias-Roman, S., Yanez-Mo, M., SanchezMadrid, F. and Arroyo, A.G. (2002) ECM regulates MT1-MMP localization with $\beta 1$ or $\alpha \mathrm{v} \beta 3$ integrins at distinct cell compartments modulating its internalization and activity on human endothelial cells. J Cell Biol, 159: 509-21.

Gao, C., Mao, S., Lo, C.H., Wirsching, P., Lerner, R.A. and Janda, K.D. (1999) Making artificial antibodies: a format for phage display of combinatorial heterodimeric arrays. Proc Natl Acad Sci U S A, 96: 6025-30.

Gaudin, P., Berthier, S., Barro, C., Zaoui, P. and Morel, F. (1997) Proteolytic potential of human neutrophil membranes. Eur J Cell Biol, 72: 345-51.

Gearing, A.J., Beckett, P., Christodoulou, M., Churchill, M., Clements, J., Davidson, A.H., Drummond, A.H., Galloway, W.A., Gilbert, R., Gordon, J.L. and et al. (1994) Processing of tumour necrosis factor- $\alpha$ precursor by metalloproteinases. Nature, 370: 555-7.

Gehrmann, M., Briknarova, K., Banyai, L., Patthy, L. and Llinas, M. (2002) The col-1 module of human matrix metalloproteinase-2 (MMP-2): structural/functional relatedness between gelatin-binding fibronectin type II modules and lysine-binding kringle domains. Biol Chem, 383: 137-48.

Ghersi, G., Dong, H., Goldstein, L.A., Yeh, Y., Häkkinen, L., Larjava, H.S. and Chen, W.T. (2002) Regulation of fibroblast migration on collagenous matrix by a cell surface peptidase complex. J Biol Chem, 277: 2923141. 
Ghosh, A.K., Ribolla, P.E. and Jacobs-Lorena, M. (2001) Targeting Plasmodium ligands on mosquito salivary glands and midgut with a phage display peptide library. Proc Natl Acad Sci U S A, 98: 13278-81.

Giancotti, F.G. and Ruoslahti, E. (1999) Integrin signaling. Science, 285: 1028-32.

Giannelli, G., Falk-Marzillier, J., Schiraldi, O., StetlerStevenson, W.G. and Quaranta, V. (1997) Induction of cell migration by matrix metalloprotease-2 cleavage of laminin-5. Science, 277: 225-8.

Ginestra, A., Monea, S., Seghezzi, G., Dolo, V., Nagase, H., Mignatti, P. and Vittorelli, M.L. (1997) Urokinase plasminogen activator and gelatinases are associated with membrane vesicles shed by human HT1080 fibrosarcoma cells. J Biol Chem, 272: 17216-22.

Glucksman, M.J., Bhattacharjee, S. and Makowski, L. (1992) Three-dimensional structure of a cloning vector. X-ray diffraction studies of filamentous bacteriophage M13 at 7 A resolution. J Mol Biol, 226: 455-70.

Goldberg, G.I., Strongin, A., Collier, I.E., Genrich, L.T. and Marmer, B.L. (1992) Interaction of 92-kDa type IV collagenase with the tissue inhibitor of metalloproteinases prevents dimerization, complex formation with interstitial collagenase, and activation of the proenzyme with stromelysin. J Biol Chem, 267: 4583-91.

Gomis-Ruth, F. (2004) Hemopexin domains. In Messerschmidt, A., Bode, W. and Cygler, M. (eds.), Handbook of Metalloproteins. John Wiley \& Sons, Chichester, Vol. 3, p. 631-646.

Goodson, R.J., Doyle, M.V., Kaufman, S.E. and Rosenberg, S. (1994) High-affinity urokinase receptor antagonists identified with bacteriophage peptide display. Proc Nat Acad Sci U S A, 91: 7129-33.

Gram, H., Schmitz, R., Zuber, J.F. and Baumann, G. (1997) Identification of phosphopeptide ligands for the Srchomology $2(\mathrm{SH} 2)$ domain of $\mathrm{Grb} 2$ by phage display. Eur J Biochem, 246: 633-7.

Grams, F., Brandstetter, H., D'Alo, S., Geppert, D., Krell, H.W., Leinert, H., Livi, V., Menta, E., Oliva, A. Zimmermann, G., Gram, F. and Livi, V.E. (2001) Pyrimidine-2,4,6-Triones: a new effective and selective class of matrix metalloproteinase inhibitors. Biol Chem, 382: $1277-85$

Greenwood, J., Willis, A.E. and Perham, R.N. (1991) Multiple display of foreign peptides on a filamentous bacteriophage. Peptides from Plasmodium falciparum circumsporozoite protein as antigens. J Mol Biol, 220 821-7.

Gress, T.M., Wallrapp, C., Frohme, M., Muller-Pillasch, F., Lacher, U., Friess, H., Buchler, M., Adler, G. and Hoheisel, J.D. (1997) Identification of genes with specific expression in pancreatic cancer by cDNA representational difference analysis. Genes Chromosomes Cancer, 19: 97-103.

Gu, Z., Kaul, M., Yan, B., Kridel, S.J., Cui, J., Strongin, A., Smith, J.W., Liddington, R.C. and Lipton, S.A. (2002) S-nitrosylation of matrix metalloproteinases: signaling pathway to neuronal cell death. Science, 297: 1186-90.

Guo, H., Li, R., Zucker, S. and Toole, B.P. (2000) EMMPRIN (CD147), an inducer of matrix metalloproteinase synthesis, also binds interstitial collagenase to the tumor cell surface. Cancer Res, 60: 888-91.

Haas, T.L., Davis, S.J. and Madri, J.A. (1998) Threedimensional type I collagen lattices induce coordinate expression of matrix metalloproteinases MT1-MMP and MMP-2 in microvascular endothelial cells. J Biol Chem, 273: $3604-10$

Hagen, D.S., Weiner, J.H. and Sykes, B.D. (1978) Fluorotyrosine M13 coat protein: fluorine-19 nuclear magnetic resonance study of the motional properties of an integral membrane protein in phospholipid vesicles. Biochemistry, 17: 3860-6.

Hagen, D.S., Weiner, J.H. and Sykes, B.D. (1979) Investigation of solvent accessibility of the fluorotyrosyl residues of $\mathrm{M} 13$ coat protein in deoxycholate micelles and phospholipid vesicles. Biochemistry, 18: 2007-12

Hahn-Dantona, E., Ruiz, J.F., Bornstein, P. and Strickland,

D.K. (2001) The low density lipoprotein receptor-related protein modulates levels of matrix metalloproteinase 9 (MMP-9) by mediating its cellular catabolism. J Biol Chem, 276: 15498-503.

Hall, A. (1994) Small GTP-binding proteins and the regulation of the actin cytoskeleton. Annu Rev Cell Biol, 10: 31-54.

Hamano, Y., Zeisberg, M., Sugimoto, H., Lively, J.C., Maeshima, Y., Yang, C., Hynes, R.O., Werb, Z. Sudhakar, A. and Kalluri, R. (2003) Physiological levels of tumstatin, a fragment of collagen IV $\alpha 3$ chain, are generated by MMP-9 proteolysis and suppress angiogenesis via avß3 integrin. Cancer Cell, 3: 589601.

Hammes, H.P., Brownlee, M., Jonczyk, A., Sutter, A. and Preissner, K.T. (1996) Subcutaneous injection of a cyclic peptide antagonist of vitronectin receptor-type integrins inhibits retinal neovascularization. Nat Med, 2: 529-33.

Han, Y.P., Nien, Y.D. and Garner, W.L. (2002) Tumor necrosis factor-a-induced proteolytic activation of promatrix metalloproteinase- 9 by human skin is controlled by down-regulating tissue inhibitor of metalloproteinase-1 and mediated by tissue-associated chymotrypsin-like proteinase. J Biol Chem, 277: 27319 27.

Hangai, M., Kitaya, N., Xu, J., Chan, C.K., Kim, J.J., Werb, Z., Ryan, S.J. and Brooks, P.C. (2002) Matrix metalloproteinase-9-dependent exposure of a cryptic migratory control site in collagen is required before retinal angiogenesis. Am J Pathol, 161: 1429-37.

Haq, M., Shafii, A., Zervos, E.E. and Rosemurgy, A.S. (2000) Addition of matrix metalloproteinase inhibition to conventional cytotoxic therapy reduces tumor implantation and prolongs survival in a murine model of human pancreatic cancer. Cancer Res, 60: 3207-11.

Healy, J.M., Murayama, O., Maeda, T., Yoshino, K. Sekiguchi, K. and Kikuchi, M. (1995) Peptide ligands for integrin $\alpha v \beta 3$ selected from random phage display libraries. Biochemistry, 34: 3948-55.

Heissig, B., Hattori, K., Dias, S., Friedrich, M., Ferris, B., Hackett, N.R., Crystal, R.G., Besmer, P., Lyden, D., Moore, M.A., Werb, Z. and Rafii, S. (2002) Recruitment of stem and progenitor cells from the bone marrow niche requires MMP-9 mediated release of kit-ligand. Cell, 109: 625-37.

Hendrickson, W.A., Horton, J.R. and LeMaster, D.M. (1990) Selenomethionyl proteins produced for analysis by multiwavelength anomalous diffraction (MAD): a vehicle for direct determination of three-dimensional structure. EMBO J, 9: 1665-72.

Herman, M.P., Sukhova, G.K., Kisiel, W., Foster, D., Kehry, M.R., Libby, P. and Schonbeck, U. (2001) Tissue factor pathway inhibitor-2 is a novel inhibitor of matrix metalloproteinases with implications for atherosclerosis. J Clin Invest, 107: 1117-26.

Hibbs, M.S., Hasty, K.A., Seyer, J.M., Kang, A.H. and Mainardi, C.L. (1985) Biochemical and immunological characterization of the secreted forms of human neutrophil gelatinase. J Biol Chem, 260: 2493-500.

Hidalgo, M. and Eckhardt, S.G. (2001) Development of matrix metalloproteinase inhibitors in cancer therapy. $J$ Natl Cancer Inst, 93: 178-93.

Higazi, A.A., Aziza, R., Samara, A.A. and Mayer, M. (1994) Regulation of fibrinolysis by non-esterified fatty acids. Biochem J, 300: 251-5.

Hill, P.A., Docherty, A.J., Bottomley, K.M., O'Connell, J.P., Morphy, J.R., Reynolds, J.J. and Meikle, M.C. (1995) Inhibition of bone resorption in vitro by selective inhibitors of gelatinase and collagenase. Biochem J, 308: 167-75.

Hipps, D.S., Hembry, R.M., Docherty, A.J., Reynolds, J.J. and Murphy, G. (1991) Purification and characterization 
of human 72-kDa gelatinase (type IV collagenase). Use of immunolocalisation to demonstrate the noncoordinate regulation of the $72-\mathrm{kDa}$ and $95-\mathrm{kDa}$ gelatinases by human fibroblasts. Biol Chem Hoppe Seyler, 372: 287-96.

Hiratsuka, S., Nakamura, K., Iwai, S., Murakami, M., Itoh, T., Kijima, H., Shipley, J.M., Senior, R.M. and Shibuya, M. (2002) MMP9 induction by vascular endothelial growth factor receptor-1 is involved in lung-specific metastasis. Cancer Cell, 2: 289-300.

Ho, A.T., Voura, E.B., Soloway, P.D., Watson, K.L. and Khokha, R. (2001) MMP inhibitors augment fibroblast adhesion through stabilization of focal adhesion contacts and up-regulation of cadherin function. $\mathrm{J} \mathrm{Biol}$ Chem, 276: 40215-24.

Hoekstra, R., Eskens, F.A. and Verweij, J. (2001) Matrix metalloproteinase inhibitors: current developments and future perspectives. Oncologist, 6: 415-27.

Holmbeck, K., Bianco, P., Caterina, J., Yamada, S., Kromer, M., Kuznetsov, S.A., Mankani, M., Robey, P.G., Poole, A.R., Pidoux, I., Ward, J.M. and Birkedal-Hansen, $H$. (1999) MT1-MMP-deficient mice develop dwarfism, osteopenia, arthritis, and connective tissue disease due to inadequate collagen turnover. Cell, 99: 81-92.

Hoyer-Hansen, G., Ronne, E., Solberg, H., Behrendt, N., Ploug, M., Lund, L.R., Ellis, V. and Dano, K. (1992) Urokinase plasminogen activator cleaves its cell surface receptor releasing the ligand-binding domain. $J$ Biol Chem, 267: 18224-9.

Hsia, D.A., Mitra, S.K., Hauck, C.R., Streblow, D.N., Nelson, J.A., Ilic, D., Huang, S., Li, E., Nemerow, G.R., Leng, J., Spencer, K.S., Cheresh, D.A. and Schlaepfer, D.D. (2003) Differential regulation of cell motility and invasion by FAK. J Cell Biol, 160: 753-67.

Hsieh, K.H., Needleman, P. and Marshall, G.R. (1987) Longacting angiotensin II inhibitors containing hexafluorovaline in position 8. J Med Chem, 30: 1097100.

Hua, J. and Muschel, R.J. (1996) Inhibition of matrix metalloproteinase 9 expression by a ribozyme blocks metastasis in a rat sarcoma model system. Cancer Res, 56: 5279-84.

Huang, S., Stupack, D., Liu, A., Cheresh, D. and Nemerow, G.R. (2000) Cell growth and matrix invasion of EBVimmortalized human $B$ lymphocytes is regulated by expression of av integrins. Oncogene, 19: 1915-23.

Hufton, S.E., Moerkerk, P.T., Meulemans, E.V., de Bruine, A., Arends, J.W. and Hoogenboom, H.R. (1999) Phage display of cDNA repertoires: the $\mathrm{pVI}$ display system and its applications for the selection of immunogenic ligands. J Immunol Methods, 231: 39-51.

Huhtala, P., Chow, L.T. and Tryggvason, K. (1990) Structure of the human type IV collagenase gene. J Biol Chem, 265: 11077-82.

Huhtala, P., Humphries, M.J., McCarthy, J.B., Tremble, P.M., Werb, Z. and Damsky, C.H. (1995) Cooperative signaling by $\alpha 5 \beta 1$ and $\alpha 4 \beta 1$ integrins regulates metalloproteinase gene expression in fibroblasts adhering to fibronectin. J Cell Biol, 129: 867-79.

Huhtala, P., Tuuttila, A., Chow, L.T., Lohi, J., Keski-Oja, J. and Tryggvason, K. (1991) Complete structure of the human gene for 92-kDa type IV collagenase. Divergent regulation of expression for the 92- and 72-kilodalton enzyme genes in HT-1080 cells. J Biol Chem, 266: 16485-90.

Hyde-DeRuyscher, R., Paige, L.A., Christensen, D.J., HydeDeRuyscher, N., Lim, A., Fredericks, Z.L., Kranz, J., Gallant, P., Zhang, J., Rocklage, S.M., Fowlkes, D.M., Wendler, P.A. and Hamilton, P.T. (2000) Detection of small-molecule enzyme inhibitors with peptides isolated from phage-displayed combinatorial peptide libraries. Chem Biol, 7: 17-25.

Hynes, R.O. (2002) Integrins: bidirectional, allosteric signaling machines. Cell, 110: 673-87.

Hynes, R.O. (2002) A reevaluation of integrins as regulators of angiogenesis. Nat Med, 8: 918-21.
Ibba, M. and Hennecke, H. (1995) Relaxing the substrate specificity of an aminoacyl-tRNA synthetase allows in vitro and in vivo synthesis of proteins containing unnatural amino acids. FEBS Lett, 364: 272-5.

Imai, K., Hiramatsu, A., Fukushima, D., Pierschbacher, M.D. and Okada, Y. (1997) Degradation of decorin by matrix metalloproteinases: identification of the cleavage sites, kinetic analyses and transforming growth factor- $\beta 1$ release. Biochem J, 322: 809-14.

Imai, K., Shikata, H. and Okada, Y. (1995) Degradation of vitronectin by matrix metalloproteinases-1, -2, -3, -7 and -9. FEBS Lett, 369: 249-51.

Itoh, T., Tanioka, M., Matsuda, H., Nishimoto, H., Yoshioka, T., Suzuki, R. and Uehira, M. (1999) Experimental metastasis is suppressed in MMP-9-deficient mice. Clin Exp Metastasis, 17: 177-81.

Itoh, T., Tanioka, M., Yoshida, H., Yoshioka, T., Nishimoto, $\mathrm{H}$. and Itohara, S. (1998) Reduced angiogenesis and tumor progression in gelatinase A-deficient mice. Cancer Res, 58: 1048-51.

Iwasa, S., Jin, X., Okada, K., Mitsumata, M. and Ooi, A. (2003) Increased expression of seprase, a membranetype serine protease, is associated with lymph node metastasis in human colorectal cancer. Cancer Lett, 199: $91-8$

Ivaska, J., Käpylä, J., Pentikäinen, O., Hoffren, A.M. Hermonen, J., Huttunen, P., Johnson, M.S. and Heino, J. (1999) A peptide inhibiting the collagen binding function of integrin $\alpha 2$ I domain. J Biol Chem, 274 3513-21.

Jaffe, A.B. and Hall, A. (2002) Rho GTPases in transformation and metastasis. Adv Cancer Res, 84: 57-80.

Jiang, A., Lehti, K., Wang, X., Weiss, S.J., Keski-Oja, J. and Pei, D. (2001) Regulation of membrane-type matrix metalloproteinase 1 activity by dynamin-mediated endocytosis. Proc Natl Acad Sci U S A, 98: 13693-8. Epub 2001 Nov 6.

Jiang, M.C., Liao, C.F. and Lee, P.H. (2001) Aspirin inhibits matrix metalloproteinase- 2 activity, increases $E$ cadherin production, and inhibits in vitro invasion of tumor cells. Biochem Biophys Res Commun, 282: 6717.

Jiang, Y., Wang, M., Celiker, M.Y., Liu, Y.E., Sang, Q.X., Goldberg, I.D. and Shi, Y.E. (2001) Stimulation of mammary tumorigenesis by systemic tissue inhibitor of matrix metalloproteinase 4 gene delivery. Cancer Res, 61: $2365-70$.

Johansson, S. and Smedsrod, B. (1986) Identification of a plasma gelatinase in preparations of fibronectin. J Biol Chem, 261: 4363-6.

Joyce, J.A., Baruch, A., Chehade, K., Meyer-Morse, N., Giraudo, E., Tsai, F.Y., Greenbaum, D.C., Hager, J.H., Bogyo, M. and Hanahan, D. (2004) Cathepsin cysteine proteases are effectors of invasive growth and angiogenesis during multistage tumorigenesis. Cancer Cell, 5: 443-53.

Kajita, M., Itoh, Y., Chiba, T., Mori, H., Okada, A., Kinoh, H. and Seiki, M. (2001) Membrane-type 1 matrix metalloproteinase cleaves CD44 and promotes cell migration. J Cell Biol, 153: 893-904.

Kane, P.M., Yamashiro, C.T., Wolczyk, D.F., Neff, N., Goebl, M. and Stevens, T.H. (1990) Protein splicing converts the yeast TFP1 gene product to the 69-kD subunit of the vacuolar $\mathrm{H}(+)$-adenosine triphosphatase. Science, 250: 651-7.

Kang, Y., Siegel, P.M., Shu, W., Drobnjak, M., Kakonen, S.M., Cordon-Cardo, C., Guise, T.A. and Massague, J. (2003) A multigenic program mediating breast cancer metastasis to bone. Cancer Cell, 3: 537-49.

Kay, B., Kurakin, A. and Hyde-DeRuyscher, R. (1998) From peptides to drugs via phage display. Drug Discovery Today, 3: 370-378.

Kay, B.K. and Hamilton, P.T. (2001) Identification of enzyme inhibitors from phage-displayed combinatorial peptide 
libraries. Comb Chem High Throughput Screen, 4: 53543.

Kenny, C.H., Ding, W., Kelleher, K., Benard, S., Dushin, E.G., Sutherland, A.G., Mosyak, L., Kriz, R. and Ellestad, G. (2003) Development of a fluorescence polarization assay to screen for inhibitors of the FtsZ/ZipA interaction. Anal Biochem, 323: 224-33.

Kiick, K.L., Saxon, E., Tirrell, D.A. and Bertozzi, C.R. (2002) Incorporation of azides into recombinant proteins for chemoselective modification by the Staudinger ligation. Proc Natl Acad Sci U S A, 99: 19-24.

Kiick, K.L., van Hest, J.C. and Tirrell, D.A. (2000) Expanding the Scope of Protein Biosynthesis by Altering the Methionyl-tRNA Synthetase Activity of a Bacterial Expression Host. Angew Chem Int Ed Engl, 39: 21482152.

Kiick, K.L., Weberskirch, R. and Tirrell, D.A. (2001) Identification of an expanded set of translationally active methionine analogues in Escherichia coli. FEBS Lett, 502: 25-30.

Kim, J., Yu, W., Kovalski, K. and Ossowski, L. (1998) Requirement for specific proteases in cancer cell intravasation as revealed by a novel semiquantitative PCR-based assay. Cell, 94: 353-62.

Kim, Y.M., Jang, J.W., Lee, O.H., Yeon, J., Choi, E.Y., Kim, K.W., Lee, S.T. and Kwon, Y.G. (2000) Endostatin inhibits endothelial and tumor cellular invasion by blocking the activation and catalytic activity of matrix metalloproteinase. Cancer Res, 60: 5410-3.

Kirshenbaum, K., Carrico, I.S. and Tirrell, D.A. (2002) Biosynthesis of proteins incorporating a versatile set of phenylalanine analogues. Chembiochem, 3: 235-7.

Klemke, R.L., Yebra, M., Bayna, E.M. and Cheresh, D.A. (1994) Receptor tyrosine kinase signaling required for integrin avß5-directed cell motility but not adhesion on vitronectin. J Cell Biol, 127: 859-66.

Knauper, V., Docherty, A.J., Smith, B., Tschesche, H. and Murphy, G. (1997) Analysis of the contribution of the hinge region of human neutrophil collagenase (HNC, MMP-8) to stability and collagenolytic activity by alanine scanning mutagenesis. FEBS Lett, 405: 60-4.

Knauper, V., Smith, B., Lopez-Otin, C. and Murphy, G. (1997) Activation of progelatinase B (proMMP-9) by active collagenase-3 (MMP-13). Eur J Biochem, 248: 369-73.

Knauper, V., Will, H., Lopez-Otin, C., Smith, B., Atkinson, S.J., Stanton, H., Hembry, R.M. and Murphy, G. (1996) Cellular mechanisms for human procollagenase-3 (MMP-13) activation. Evidence that MT1-MMP (MMP14) and gelatinase a (MMP-2) are able to generate active enzyme. J Biol Chem, 271: 17124-31.

Koivunen, E., Arap, W., Valtanen, H., Rainisalo, A., Medina, O.P., Heikkilä, P., Kantor, C., Gahmberg, C.G., Salo, T., Konttinen, Y.T., Sorsa, T., Ruoslahti, E. and Pasqualini, R. (1999) Tumor targeting with a selective gelatinase inhibitor. Nat Biotechnol, 17: 768-74.

Koivunen, E., Gay, D.A. and Ruoslahti, E. (1993) Selection of peptides binding to the $\alpha 5 \beta 1$ integrin from phage display library. J Biol Chem, 268: 20205-10.

Koivunen, E., Huhtala, M.L. and Stenman, U.H. (1989) Human ovarian tumor-associated trypsin. Its purification and characterization from mucinous cyst fluid and identification as an activator of pro-urokinase. J Biol Chem, 264: 14095-9.

Koivunen, E., Ranta, T.M., Annila, A., Taube, S., Uppala, A., Jokinen, M., van Willigen, G., Ihanus, E. and Gahmberg, C.G. (2001) Inhibition of $\beta 2$ integrinmediated leukocyte cell adhesion by leucine-leucineglycine motif-containing peptides. J Cell Biol, 153: 90516.

Koivunen, E., Restel, B.H., Rajotte, D., Lahdenranta, J., Hagedorn, M., Arap, W. and Pasqualini, R. (1999) Integrin-binding peptides derived from phage display libraries. Methods Mol Biol, 129: 3-17.

Koivunen, E., Wang, B. and Ruoslahti, E. (1995) Phage libraries displaying cyclic peptides with different ring sizes: ligand specificities of the RGD-directed integrins. Biotechnology (N Y), 13: 265-70.

Koolwijk, P., Sidenius, N., Peters, E., Sier, C.F., Hanemaaijer, R., Blasi, F. and van Hinsbergh, V.W. (2001) Proteolysis of the urokinase-type plasminogen activator receptor by metalloproteinase-12: implication for angiogenesis in fibrin matrices. Blood, 97: 3123-31.

Koop, S., Khokha, R., Schmidt, E.E., MacDonald, I.C., Morris, V.L., Chambers, A.F. and Groom, A.C. (1994) Overexpression of metalloproteinase inhibitor in B16F10 cells does not affect extravasation but reduces tumor growth. Cancer Res, 54: 4791-7.

Koop, S., Schmidt, E.E., MacDonald, I.C., Morris, V.L., Khokha, R., Grattan, M., Leone, J., Chambers, A.F. and Groom, A.C. (1996) Independence of metastatic ability and extravasation: metastatic ras-transformed and control fibroblasts extravasate equally well. Proc Natl Acad Sci U S A, 93: 11080-4.

Kotra, L.P., Zhang, L., Fridman, R., Orlando, R. and Mobashery, S. (2002) N-Glycosylation pattern of the zymogenic form of human matrix metalloproteinase-9. Bioorg Chem, 30: 356-70.

Kratz, F., Drevs, J., Bing, G., Stockmar, C., Scheuermann, K., Lazar, P. and Unger, C. (2001) Development and in vitro efficacy of novel MMP2 and MMP9 specific doxorubicin albumin conjugates. Bioorg Med Chem Lett, 11: 2001-6.

Kridel, S.J., Chen, E., Kotra, L.P., Howard, E.W., Mobashery, S. and Smith, J.W. (2001) Substrate hydrolysis by matrix metalloproteinase-9. J Biol Chem, 276: 20572-8.

Kridel, S.J., Sawai, H., Ratnikov, B.I., Chen, E.I., Li, W., Godzik, A., Strongin, A.Y. and Smith, J.W. (2002) A unique substrate binding mode discriminates membrane type-1 matrix metalloproteinase from other matrix metalloproteinases. J Biol Chem, 277: 23788-93.

Kuper, H., Adami, H.O. and Trichopoulos, D. (2000) Infections as a major preventable cause of human cancer. J Intern Med, 248: 171-83.

Kupferman, M.E., Fini, M.E., Muller, W.J., Weber, R., Cheng, Y. and Muschel, R.J. (2000) Matrix metalloproteinase 9 promoter activity is induced coincident with invasion during tumor progression. Am J Pathol, 157: 1777-83.

Kuwabara, I., Maruyama, H., Mikawa, Y.G., Zuberi, R.I., Liu, F.T. and Maruyama, I.N. (1997) Efficient epitope mapping by bacteriophage lambda surface display. Nat Biotechnol, 15: 74-8.

Kwan, J.A., Schulze, C.J., Wang, W., Leon, H., Sariahmetoglu, M., Sung, M., Sawicka, J., Sims, D.E., Sawicki, G. and Schulz, R. (2004) Matrix metalloproteinase-2 (MMP-2) is present in the nucleus of cardiac myocytes and is capable of cleaving poly (ADP-ribose) polymerase (PARP) in vitro. FASEB J, 18: 690-2.

Kwon, I., Kirshenbaum, K. and Tirrell, D.A. (2003) Breaking the degeneracy of the genetic code. J Am Chem Soc, 125: $7512-3$.

Laakkonen, P., Porkka, K., Hoffman, J.A. and Ruoslahti, E. (2002) A tumor-homing peptide with a targeting specificity related to lymphatic vessels. Nat Med, 8: 751-5.

Lakka, S.S., Rajan, M., Gondi, C., Yanamandra, N., Chandrasekar, N., Jasti, S.L., Adachi, Y., Siddique, K., Gujrati, M., Olivero, W., Dinh, D.H., Kouraklis, G., Kyritsis, A.P. and Rao, J.S. (2002) Adenovirusmediated expression of antisense MMP-9 in glioma cells inhibits tumor growth and invasion. Oncogene, 21: 8011-9.

Lamberg, A., Nieminen, S., Qiao, M. and Savilahti, H. (2002) Efficient insertional mutagenesis strategy for bacterial genomes involving electroporation of in vitroassembled DNA transposition complexes of bacteriophage Mu. Appl. Environ. Microbiol., 68: 705712. 
Larjava, H., Lyons, J.G., Salo, T., Mäkelä, M., Koivisto, L. Birkedal-Hansen, H., Akiyama, S.K., Yamada, K.M. and Heino, J. (1993) Anti-integrin antibodies induce type IV collagenase expression in keratinocytes. J Cell Physiol, 157: $190-200$

Larsen, P.H., Wells, J.E., Stallcup, W.B., Opdenakker, G. and Yong, V.W. (2003) Matrix metalloproteinase-9 facilitates remyelination in part by processing the inhibitory NG2 proteoglycan. J Neurosci, 23: 11127-35.

Lauffenburger, D.A. and Horwitz, A.F. (1996) Cell migration: a physically integrated molecular process. Cell, 84: 359-69.

Lee, A.Y., Akers, K.T., Collier, M., Li, L., Eisen, A.Z. and Seltzer, J.L. (1997) Intracellular activation of gelatinase A (72-kDa type IV collagenase) by normal fibroblasts. Proc Natl Acad SciU S A, 94: 4424-9.

Legrand, C., Gilles, C., Zahm, J.M., Polette, M., Buisson, A.C., Kaplan, H., Birembaut, P. and Tournier, J.M. (1999) Airway epithelial cell migration dynamics. MMP9 role in cell-extracellular matrix remodeling. $J$ Cell Biol, 146: $517-29$.

Lehti, K., Lohi, J., Valtanen, H. and Keski-Oja, J. (1998) Proteolytic processing of membrane-type-1 matrix metalloproteinase is associated with gelatinase $A$ activation at the cell surface. Biochem J, 334: 345-53.

Lehti, K., Lohi, J., Juntunen, M.M., Pei, D. and Keski-Oja, J. (2002) Oligomerization through hemopexin and cytoplasmic domains regulates the activity and turnover of membrane-type 1 matrix metalloproteinase. J Biol Chem, 277: 8440-8.

Lelongt, B., Bengatta, S., Delauche, M., Lund, L.R., Werb, Z. and Ronco, P.M. (2001) Matrix metalloproteinase 9 protects mice from anti-glomerular basement membrane nephritis through its fibrinolytic activity. $J$ Exp Med, 193: 793-802.

LePage, R.N., Fosang, A.J., Fuller, S.J., Murphy, G., Evin, G., Beyreuther, K., Masters, C.L. and Small, D.H. (1995) Gelatinase A possesses a $\beta$-secretase-like activity in cleaving the amyloid protein precursor of Alzheimer's disease. FEBS Lett, 377: 267-70.

Lesaicherre, M.L., Lue, R.Y., Chen, G.Y., Zhu, Q. and Yao, S.Q. (2002) Intein-mediated biotinylation of proteins and its application in a protein microarray. J Am Chem Soc, 124: 8768-9.

Levi, E., Fridman, R., Miao, H.Q., Ma, Y.S., Yayon, A. and Vlodavsky, I. (1996) Matrix metalloproteinase 2 releases active soluble ectodomain of fibroblast growth factor receptor 1. Proc Natl Acad Sci U S A, 93: 706974.

Leytus, S.P., Loeb, K.R., Hagen, F.S., Kurachi, K. and Davie, E.W. (1988) A novel trypsin-like serine protease (hepsin) with a putative transmembrane domain expressed by human liver and hepatoma cells. Biochemistry, 27: 1067-74.

Li, S. and Roberts, R.W. (2003) A novel strategy for in vitro selection of Peptide-drug conjugates. Chem Biol, 10: 233-9.

Li, W.P. and Anderson, C.J. (2003) Imaging matrix metalloproteinase expression in tumors. Q J Nucl Med, 47: 201-8.

Lien, S. and Lowman, H.B. (2003) Therapeutic peptides. Trends Biotechnol, 21: 556-62.

Lijnen, H.R., Silence, J., Van Hoef, B. and Collen, D. (1998) Stromelysin-1 (MMP-3)-independent gelatinase expression and activation in mice. Blood, 91: 2045-53.

Lin, R., Cerione, R.A. and Manor, D. (1999) Specific contributions of the small GTPases Rho, Rac, and Cdc42 to Dbl transformation. J Biol Chem, 274: 2363341

Link, A.J., Mock, M.L. and Tirrell, D.A. (2003) Non-canonical amino acids in protein engineering. Curr Opin Biotechnol, 14: 603-9.

Liou, T.G. and Campbell, E.J. (1996) Quantum proteolysis resulting from release of single granules by human neutrophils: a novel, nonoxidative mechanism of extracellular proteolytic activity. J Immunol, 157: 262431

Liu, D.R. and Schultz, P.G. (1999) Progress toward the evolution of an organism with an expanded genetic code. Proc Natl Acad SciU S A, 96: 4780-5.

Liu, H., Wang, L., Brock, A., Wong, C.H. and Schultz, P.G. (2003) A method for the generation of glycoprotein mimetics. J Am Chem Soc, 125: 1702-3.

Liu, J., Tsao, M.S., Pagura, M., Shalinsky, D.R., Khoka, R., Fata, J. and Johnston, M.R. (2003) Early combined treatment with carboplatin and the MMP inhibitor, prinomastat, prolongs survival and reduces systemic metastasis in an aggressive orthotopic lung cancer model. Lung Cancer, 42: 335-44.

Liu, L.T., Chang, H.C., Chiang, L.C. and Hung, W.C. (2002) Induction of RECK by nonsteroidal anti-inflammatory drugs in lung cancer cells. Oncogene, 21: 8347-50.

Liu, Z., Zhou, X., Shapiro, S.D., Shipley, J.M., Twining, S.S., Diaz, L.A., Senior, R.M. and Werb, Z. (2000) The serpin a1-proteinase inhibitor is a critical substrate for gelatinase B/MMP-9 in vivo. Cell, 102: 647-55.

Lowman, H.B., Bass, S.H., Simpson, N. and Wells, J.A. (1991) Selecting high-affinity binding proteins by monovalent phage display. Biochemistry, 30: 10832-8.

Luttun, A., Lutgens, E., Manderveld, A., Maris, K., Collen, D., Carmeliet, P. and Moons, L. (2004) Loss of matrix metalloproteinase- 9 or matrix metalloproteinase- 12 protects apolipoprotein E-deficient mice against atherosclerotic media destruction but differentially affects plaque growth. Circulation, 109: 1408-14.

Maeshima, Y., Yerramalla, U.L., Dhanabal, M., Holthaus, K.A., Barbashov, S., Kharbanda, S., Reimer, C., Manfredi, M., Dickerson, W.M. and Kalluri, R. (2001) Extracellular matrix-derived peptide binds to av $\beta 3$ integrin and inhibits angiogenesis. J Biol Chem, 276: 31959-68.

Magee, J.A., Araki, T., Patil, S., Ehrig, T., True, L., Humphrey, P.A., Catalona, W.J., Watson, M.A. and Milbrandt, J. (2001) Expression profiling reveals hepsin overexpression in prostate cancer. Cancer Res, 61 5692-6.

Magliery, T.J., Anderson, J.C. and Schultz, P.G. (2001) Expanding the genetic code: selection of efficient suppressors of four-base codons and identification of "shifty" four-base codons with a library approach in Escherichia coli. J Mol Biol, 307: 755-69.

Mai, J., Finley, R.L., Jr., Waisman, D.M. and Sloane, B.F. (2000) Human procathepsin B interacts with the annexin II tetramer on the surface of tumor cells. J Biol Chem, 275: 12806-12.

Mäkelä, M., Salo, T. and Larjava, H. (1998) MMP-9 from TNF $\alpha$-stimulated keratinocytes binds to cell membranes and type I collagen: a cause for extended matrix degradation in inflammation? Biochem Biophys Res Commun, 253: 325-35.

Makowski, L. (1992) Terminating a macromolecular helix. Structural model for the minor proteins of bacteriophage M13. J Mol Biol, 228: 885-92.

Manes, S., Llorente, M., Lacalle, R.A., Gomez-Mouton, C., Kremer, L., Mira, E. and Martinez, A.C. (1999) The matrix metalloproteinase-9 regulates the insulin-like growth factor-triggered autocrine response in DU-145 carcinoma cells. J Biol Chem, 274: 6935-45.

Mansour, A.M., Drevs, J., Esser, N., Hamada, F.M., Badary, O.A., Unger, C., Fichtner, I. and Kratz, F. (2003) A new approach for the treatment of malignant melanoma: enhanced antitumor efficacy of an albumin-binding doxorubicin prodrug that is cleaved by matrix metalloproteinase 2. Cancer Res, 63: 4062-6.

Mao, C., Solis, D.J., Reiss, B.D., Kottmann, S.T., Sweeney, R.Y., Hayhurst, A., Georgiou, G., Iverson, B. and Belcher, A.M. (2004) Virus-based toolkit for the directed synthesis of magnetic and semiconducting nanowires. Science, 303: 213-7. 
Marciano, D.K., Russel, M. and Simon, S.M. (1999) An aqueous channel for filamentous phage export. Science, 284: 1516-9.

Mathys, S., Evans, T.C., Chute, I.C., Wu, H., Chong, S., Benner, J., Liu, X.Q. and Xu, M.Q. (1999) Characterization of a self-splicing mini-intein and its conversion into autocatalytic $\mathrm{N}$ - and $\mathrm{C}$-terminal cleavage elements: facile production of protein building blocks for protein ligation. Gene, 231: 1-13.

Matthews, D.J., Goodman, L.J., Gorman, C.M. and Wells, J.A. (1994) A survey of furin substrate specificity using substrate phage display. Protein Sci, 3: 1197-205.

Matthews, D.J. and Wells, J.A. (1993) Substrate phage: selection of protease substrates by monovalent phage display. Science, 260: 1113-7.

Matthews, L.J., Davis, R. and Smith, G.P. (2002) Immunogenically fit subunit vaccine components via epitope discovery from natural peptide libraries. $J$ Immunol, 169: 837-46.

Mattu, T.S., Royle, L., Langridge, J., Wormald, M.R., Van den Steen, P.E., Van Damme, J., Opdenakker, G., Harvey, D.J., Dwek, R.A. and Rudd, P.M. (2000) Oglycan analysis of natural human neutrophil gelatinase $B$ using a combination of normal phase-HPLC and online tandem mass spectrometry: implications for the domain organization of the enzyme. Biochemistry, 39: 15695-704.

Mayer, U., Mann, K., Timpl, R. and Murphy, G. (1993) Sites of nidogen cleavage by proteases involved in tissue homeostasis and remodelling. Eur J Biochem, 217: 877-84.

Mazzieri, R., Masiero, L., Zanetta, L., Monea, S., Onisto, M., Garbisa, S. and Mignatti, P. (1997) Control of type IV collagenase activity by components of the urokinaseplasmin system: a regulatory mechanism with cellbound reactants. EMBO J, 16: 2319-32.

McCafferty, J., Griffiths, A.D., Winter, G. and Chiswell, D.J. (1990) Phage antibodies: filamentous phage displaying antibody variable domains. Nature, 348: 552-4.

McCafferty, J., Jackson, R.H. and Chiswell, D.J. (1991) Phage-enzymes: expression and affinity chromatography of functional alkaline phosphatase on the surface of bacteriophage. Protein Eng, 4: 955-61.

McLafferty, M.A., Kent, R.B., Ladner, R.C. and Markland, W (1993) M13 bacteriophage displaying disulfideconstrained microproteins. Gene, 128: 29-36.

McQuibban, G.A., Butler, G.S., Gong, J.H., Bendall, L., Power, C., Clark-Lewis, I. and Overall, C.M. (2001) Matrix metalloproteinase activity inactivates the CXC chemokine stromal cell-derived factor-1. J Biol Chem 276: 43503-8.

McQuibban, G.A., Gong, J.H., Tam, E.M., McCulloch, C.A., Clark-Lewis, I. and Overall, C.M. (2000) Inflammation dampened by gelatinase A cleavage of monocyte chemoattractant protein-3. Science, 289: 1202-6.

McQuibban, G.A., Gong, J.H., Wong, J.P., Wallace, J.L., Clark-Lewis, I. and Overall, C.M. (2002) Matrix metalloproteinase processing of monocyte chemoattractant proteins generates CC chemokine receptor antagonists with anti-inflammatory properties in vivo. Blood, 100: 1160-7.

Medina, O.P., Soderlund, T., Laakkonen, L.J., Tuominen, E.K., Koivunen, E. and Kinnunen, P.K. (2001) Binding of Novel Peptide Inhibitors of Type IV Collagenases to Phospholipid Membranes and Use in Liposome Targeting to Tumor Cells in Vitro. Cancer Res, 61 3978-3985.

Mehl, R.A., Anderson, J.C., Santoro, S.W., Wang, L., Martin A.B., King, D.S., Horn, D.M. and Schultz, P.G. (2003) Generation of a bacterium with a 21 amino acid genetic code. J Am Chem Soc, 125: 935-9.

Mignatti, P. and Rifkin, D.B. (1993) Biology and biochemistry of proteinases in tumor invasion. Physiol Rev, 73: 16195.

Minks, C., Huber, R., Moroder, L. and Budisa, N. (1999) Atomic mutations at the single tryptophan residue of human recombinant annexin $\mathrm{V}$ : effects on structure, stability, and activity. Biochemistry, 38: 10649-59.

Minks, C., Huber, R., Moroder, L. and Budisa, N. (2000) Noninvasive tracing of recombinant proteins with "fluorophenylalanine-fingers". Anal Biochem, 284: 2934.

Mira, E., Manes, S., Lacalle, R.A., Marquez, G. and Martinez, A.C. (1999) Insulin-like growth factor Itriggered cell migration and invasion are mediated by matrix metalloproteinase-9. Endocrinology, 140: 165764.

Model, P. and Russel, M. (1988) Filamentous Bacteriophage. The bacteriophages. Plenum, New York, Vol. 2, pp. 375-456.

Moestrup, S.K., Holtet, T.L., Etzerodt, M., Thogersen, H.C., Nykjaer, A., Andreasen, P.A., Rasmussen, H.H., Sottrup-Jensen, L. and Gliemann, J. (1993) A 2macroglobulin-proteinase complexes, plasminogen activator inhibitor type-1-plasminogen activator complexes, and receptor-associated protein bind to a region of the $\alpha$ 2-macroglobulin receptor containing a cluster of eight complement-type repeats. J Biol Chem, 268: 13691-6.

Mohan, R., Chintala, S.K., Jung, J.C., Villar, W.V., McCabe, F., Russo, L.A., Lee, Y., McCarthy, B.E., Wollenberg, K.R., Jester, J.V., Wang, M., Welgus, H.G., Shipley, J.M., Senior, R.M. and Fini, M.E. (2002) Matrix metalloproteinase gelatinase B (MMP-9) coordinates and effects epithelial regeneration. J Biol Chem, 277: 2065-72. Epub 2001 Oct 31.

Moilanen, M., Sorsa, T., Stenman, M., Nyberg, P., Lindy, O., Vesterinen, J., Paju, A., Konttinen, Y.T., Stenman, U.H and Salo, T. (2003) Tumor-associated trypsinogen-2 (trypsinogen-2) activates procollagenases (MMP-1, -8, -13) and stromelysin-1 (MMP-3) and degrades type I collagen. Biochemistry, 42: 5414-20.

Monea, S., Lehti, K., Keski-Oja, J. and Mignatti, P. (2002) Plasmin activates pro-matrix metalloproteinase-2 with a membrane-type 1 matrix metalloproteinase-dependent mechanism. J Cell Physiol, 192: 160-70.

Monsky, W.L., Lin, C.Y., Aoyama, A., Kelly, T., Akiyama, S.K., Mueller, S.C. and Chen, W.T. (1994) A potential marker protease of invasiveness, seprase, is localized on invadopodia of human malignant melanoma cells. Cancer Res, 54: 5702-10.

Montuori, N., Carriero, M.V., Salzano, S., Rossi, G. and Ragno, P. (2002) The cleavage of the urokinase receptor regulates its multiple functions. J Biol Chem, 277: 46932-9.

Moore, B.B., Arenberg, D.A., Addison, C.L., Keane, M.P., Polverini, P.J. and Strieter, R.M. (1998) CXC chemokines mechanism of action in regulating tumor angiogenesis. Angiogenesis, 2: 123-34.

Morgunova, E., Tuuttila, A., Bergmann, U., Isupov, M., Lindqvist, Y., Schneider, G. and Tryggvason, K. (1999) Structure of human pro-matrix metalloproteinase-2: activation mechanism revealed. Science, 284: 1667-70.

Morgunova, E., Tuuttila, A., Bergmann, U. and Tryggvason, K. (2002) Structural insight into the complex formation of latent matrix metalloproteinase 2 with tissue inhibitor of metalloproteinase 2. Proc Natl Acad Sci U S A, 99: 7414-9.

Mori, H., Tomari, T., Koshikawa, N., Kajita, M., Itoh, Y., Sato H., Tojo, H., Yana, I. and Seiki, M. (2002) CD44 directs membrane-type 1 matrix metalloproteinase to lamellipodia by associating with its hemopexin-like domain. EMBO J, 21: 3949-59.

Morini, M., Mottolese, M., Ferrari, N., Ghiorzo, F., Buglioni, S., Mortarini, R., Noonan, D.M., Natali, P.G. and Albini, A. (2000) The $\alpha 3 \beta 1$ integrin is associated with mammary carcinoma cell metastasis, invasion, and gelatinase B (MMP-9) activity. Int J Cancer, 87: 336-42.

Morodomi, T., Ogata, Y., Sasaguri, Y., Morimatsu, M. and Nagase, H. (1992) Purification and characterization of matrix metalloproteinase 9 from U937 monocytic 
leukaemia and HT1080 fibrosarcoma cells. Biochem J, 285: 603-11.

Morris, V.L., Koop, S., MacDonald, I.C., Schmidt, E.E., Grattan, M., Percy, D., Chambers, A.F. and Groom, A.C. (1994) Mammary carcinoma cell lines of high and low metastatic potential differ not in extravasation but in subsequent migration and growth. Clin Exp Metastasis, 12: 357-67.

Morrison, C.J., Butler, G.S., Bigg, H.F., Roberts, C.R., Soloway, P.D. and Overall, C.M. (2001) Cellular activation of MMP-2 (gelatinase A) by MT2-MMP occurs via a TIMP-2-independent pathway. J Biol Chem, 276: 47402-10.

Mott, J.D., Thomas, C.L., Rosenbach, M.T., Takahara, K. Greenspan, D.S. and Banda, M.J. (2000) Posttranslational proteolytic processing of procollagen Cterminal proteinase enhancer releases a metalloproteinase inhibitor. J Biol Chem, 275: 1384-90.

Mueller, S.C., Ghersi, G., Akiyama, S.K., Sang, Q.X., Howard, L., Pineiro-Sanchez, M., Nakahara, H., Yeh, Y. and Chen, W.T. (1999) A novel protease-docking function of integrin at invadopodia. J Biol Chem, 274: 24947-52.

Muller, A., Homey, B., Soto, H., Ge, N., Catron, D., Buchanan, M.E., McClanahan, T., Murphy, E., Yuan, W., Wagner, S.N., Barrera, J.L., Mohar, A., Verastegui, E. and Zlotnik, A. (2001) Involvement of chemokine receptors in breast cancer metastasis. Nature, 410: 50 6.

Murashige, M., Miyahara, M., Shiraishi, N., Saito, T., Kohno K. and Kobayashi, M. (1996) Enhanced expression of tissue inhibitors of metalloproteinases in human colorectal tumors. Jpn J Clin Oncol, 26: 303-9.

Murono, S., Yoshizaki, T., Sato, H., Takeshita, H., Furukawa, M. and Pagano, J.S. (2000) Aspirin inhibits tumor cell invasiveness induced by Epstein-Barr virus latent membrane protein 1 through suppression of matrix metalloproteinase- 9 expression. Cancer Res, 60: 2555-61.

Murphy, G., Bretz, U., Baggiolini, M. and Reynolds, J.J. (1980) The latent collagenase and gelatinase of human polymorphonuclear neutrophil leucocytes. Biochem J, 192: $517-25$.

Murphy, G., Cockett, M.I., Ward, R.V. and Docherty, A.J. (1991) Matrix metalloproteinase degradation of elastin, type IV collagen and proteoglycan. A quantitative comparison of the activities of $95 \mathrm{kDa}$ and $72 \mathrm{kDa}$ gelatinases, stromelysins- 1 and -2 and punctuated metalloproteinase (PUMP). Biochem J, 277: 277-9.

Murphy, G. and Crabbe, T. (1995) Gelatinases A and B. Methods Enzymol, 248: 470-84.

Murphy-Ullrich, J.E. (2001) The de-adhesive activity of matricellular proteins: is intermediate cell adhesion an adaptive state? J Clin Invest, 107: 785-90.

Mustjoki, S., Sidenius, N., Sier, C.F., Blasi, F., Elonen, E., Alitalo, R and Vaheri, A. (2000) Soluble urokinase receptor levels correlate with number of circulating tumor cells in acute myeloid leukemia and decrease rapidly during chemotherapy. Cancer Res, 60: 7126-32.

Myöhänen, H., Virtanen, I. and Vaheri, A. (2001) Elimination of hydrocortisone from the medium enables tissue plasminogen activator gene expression by normal and immortalized nonmalignant human epithelial cells. Biol Chem, 382: 1563-73.

Nabeshima, K., Inoue, T., Shimao, Y., Okada, Y., Itoh, Y., Seiki, M. and Koono, M. (2000) Front-cell-specific expression of membrane-type 1 matrix metalloproteinase and gelatinase A during cohort migration of colon carcinoma cells induced by hepatocyte growth factor/scatter factor. Cancer Res, 60: 3364-9

Nakahara, H., Howard, L., Thompson, E.W., Sato, H., Seiki, M., Yeh, Y. and Chen, W.T. (1997)

Transmembrane/cytoplasmic domain-mediated membrane type 1-matrix metalloprotease docking to invadopodia is required for cell invasion. Proc Natl Acad Sci U S A, 94: 7959-64.

Nakamura, H., Fujii, Y., Inoki, I., Sugimoto, K., Tanzawa, K., Matsuki, H., Miura, R., Yamaguchi, Y. and Okada, Y. (2000) Brevican is degraded by matrix metalloproteinases and aggrecanase-1 (ADAMTS4) at different sites. J Biol Chem, 275: 38885-90.

Nakamura, H., Fujii, Y., Ohuchi, E., Yamamoto, E. and Okada, Y. (1998) Activation of the precursor of human stromelysin 2 and its interactions with other matrix metalloproteinases. Eur J Biochem, 253: 67-75.

Namy, O., Rousset, J.P., Napthine, S. and Brierley, I. (2004) Reprogrammed genetic decoding in cellular gene expression. Mol Cell, 13: 157-68.

Nath, D., Slocombe, P.M., Stephens, P.E., Warn, A., Hutchinson, G.R., Yamada, K.M., Docherty, A.J. and Murphy, G. (1999) Interaction of metargidin (ADAM-15) with $\alpha v \beta 3$ and $\alpha 5 \beta 1$ integrins on different haemopoietic cells. J Cell Sci, 112: 579-87.

Nathanson, S.D. (2003) Insights into the mechanisms of lymph node metastasis. Cancer, 98: 413-23.

Nelissen, I., Martens, E., Van den Steen, P.E., Proost, P., Ronsse, I. and Opdenakker, G. (2003) Gelatinase $\mathrm{B} /$ matrix metalloproteinase- 9 cleaves interferon- $\beta$ and is a target for immunotherapy. Brain, 126: 1371-81.

Nelson, A.R., Fingleton, B., Rothenberg, M.L. and Matrisian, L.M. (2000) Matrix metalloproteinases: biologic activity and clinical implications. J Clin Oncol, 18: 1135-49.

Nemeth, J.A., Rafe, A., Steiner, M. and Goolsby, C.L. (1996) TIMP-2 growth-stimulatory activity: a concentrationand cell type-specific response in the presence of insulin. Exp Cell Res, 224: 110-5.

Netzer, K.O., Suzuki, K., Itoh, Y., Hudson, B.G. and Khalifah, R.G. (1998) Comparative analysis of the noncollagenous NC1 domain of type IV collagen: identification of structural features important for assembly, function, and pathogenesis. Protein Sci, 7 1340-51.

Nguyen, M., Arkell, J. and Jackson, C.J. (2000) Activated protein $C$ directly activates human endothelial gelatinase A. J Biol Chem, 275: 9095-8.

Nguyen, Q., Murphy, G., Hughes, C.E., Mort, J.S. and Roughley, P.J. (1993) Matrix metalloproteinases cleave at two distinct sites on human cartilage link protein. Biochem J, 295: 595-8.

Niemz, A. and Tirrell, D.A. (2001) Self-association and membrane-binding behavior of melittins containing trifluoroleucine. J Am Chem Soc, 123: 7407-13

Niu, J., Gu, X., Turton, J., Meldrum, C., Howard, E.W. and Agrez, M. (1998) Integrin-mediated signalling of gelatinase B secretion in colon cancer cells. Biochem. Biophys. Res. Commun., 249: 287-91.

Noren, C.J., Anthony-Cahill, S.J., Griffith, M.C. and Schultz, P.G. (1989) A general method for site-specific incorporation of unnatural amino acids into proteins. Science, 244: 182-8.

Nowak, M.W., Kearney, P.C., Sampson, J.R., Saks, M.E., Labarca, C.G., Silverman, S.K., Zhong, W., Thorson, J., Abelson, J.N., Davidson, N. and et al. (1995) Nicotinic receptor binding site probed with unnatural amino acid incorporation in intact cells. Science, $\mathbf{2 6 8}$. 439-42.

Nyberg, P., Heikkilä, P., Sorsa, T., Luostarinen, J., Heljasvaara, R., Stenman, U.H., Pihlajaniemi, T. and Salo, T. (2003) Endostatin inhibits human tongue carcinoma cell invasion and intravasation and blocks the activation of matrix metalloprotease-2, -9 , and -13 . J Biol Chem, 278: 22404-11.

Nyberg, P., Moilanen, M., Paju, A., Sarin, A., Stenman, U.H., Sorsa, T. and Salo, T. (2002) MMP-9 activation by tumor trypsin-2 enhances in vivo invasion of human tongue carcinoma cells. J Dent Res, 81: 831-5.

Nyormoi, O., Mills, L. and Bar-Eli, M. (2003) An MMP2/MMP-9 inhibitor, 5a, enhances apoptosis induced by ligands of the TNF receptor superfamily in cancer cells. Cell Death Differ, 10: 558-69. 
Ochieng, J., Fridman, R., Nangia-Makker, P., Kleiner, D.E., Liotta, L.A., Stetler-Stevenson, W.G. and Raz, A. (1994) Galectin-3 is a novel substrate for human matrix metalloproteinases-2 and -9. Biochemistry, 33: 1410914.

O'Connell, J.P., Willenbrock, F., Docherty, A.J., Eaton, D. and Murphy, G. (1994) Analysis of the role of the $\mathrm{COOH}$-terminal domain in the activation, proteolytic activity, and tissue inhibitor of metalloproteinase interactions of gelatinase B. J Biol Chem, 269: 1496773

Ogata, Y., Enghild, J.J. and Nagase, H. (1992) Matrix metalloproteinase 3 (stromelysin) activates the precursor for the human matrix metalloproteinase 9. J Biol Chem, 267: 3581-4.

Oh, J., Takahashi, R., Adachi, E., Kondo, S., Kuratomi, S. Noma, A., Alexander, D.B., Motoda, H., Okada, A., Seiki, M., Itoh, T., Itohara, S., Takahashi, C. and Noda, M. (2004) Mutations in two matrix metalloproteinase genes, MMP-2 and MT1-MMP, are synthetic lethal in mice. Oncogene, 5: 5

Oh, J., Takahashi, R., Kondo, S., Mizoguchi, A., Adachi, E., Sasahara, R.M., Nishimura, S., Imamura, Y., Kitayama H., Alexander, D.B., Ide, C., Horan, T.P., Arakawa, T., Yoshida, H., Nishikawa, S., Itoh, Y., Seiki, M., Itohara, S., Takahashi, C. and Noda, M. (2001) The membraneanchored MMP inhibitor RECK is a key regulator of extracellular matrix integrity and angiogenesis. Cell, 107: 789-800.

Okada, Y., Morodomi, T., Enghild, J.J., Suzuki, K., Yasui, A., Nakanishi, I., Salvesen, G. and Nagase, H. (1990) Matrix metalloproteinase 2 from human rheumatoid synovial fibroblasts. Purification and activation of the precursor and enzymic properties. Eur J Biochem, 194: 721-30.

Olson, M.W., Gervasi, D.C., Mobashery, S. and Fridman, R. (1997) Kinetic analysis of the binding of human matrix metalloproteinase-2 and -9 to tissue inhibitor of metalloproteinase (TIMP)-1 and TIMP-2. J Biol Chem, 272: 29975-83.

Olson, M.W., Toth, M., Gervasi, D.C., Sado, Y., Ninomiya, Y. and Fridman, R. (1998) High affinity binding of latent matrix metalloproteinase- 9 to the $\alpha 2$ (IV) chain of collagen IV. J Biol Chem, 273: 10672-81.

O'Reilly, M.S., Boehm, T., Shing, Y., Fukai, N., Vasios, G., Lane, W.S., Flynn, E., Birkhead, J.R., Olsen, B.R. and Folkman, J. (1997) Endostatin: an endogenous inhibito of angiogenesis and tumor growth. Cell, 88: 277-85.

O'Reilly, M.S., Wiederschain, D., Stetler-Stevenson, W.G. Folkman, J. and Moses, M.A. (1999) Regulation of angiostatin production by matrix metalloproteinase- 2 in a model of concomitant resistance. J Biol Chem, 274 29568-71.

Owen, C.A., Hu, Z., Barrick, B. and Shapiro, S.D. (2003) Inducible expression of tissue inhibitor of metalloproteinases-resistant matrix metalloproteinase- 9 on the cell surface of neutrophils. Am J Respir Cell Mol Biol, 29: 283-94.

Owens, L.V., Xu, L., Craven, R.J., Dent, G.A., Weiner, T.M., Kornberg, L., Liu, E.T. and Cance, W.G. (1995) Overexpression of the focal adhesion kinase (p125FAK) in invasive human tumors. Cancer Res, 55: 2752-5.

Overall, C.M. (2002) Molecular determinants of metalloproteinase substrate specificity: matrix metalloproteinase substrate binding domains, modules, and exosites. Mol Biotechnol, 22: 51-86.

Overall, C.M. and Lopez-Otin, C. (2002) Strategies for MMP inhibition in cancer: innovations for the post-trial era. Nat Rev Cancer, 2: 657-72

Overall, C.M. and Sodek, J. (1990) Concanavalin A produces a matrix-degradative phenotype in human fibroblasts. Induction and endogenous activation of collagenase, $72-k D a$ gelatinase, and Pump-1 is accompanied by the suppression of the tissue inhibitor of matrix metalloproteinases. J Biol Chem, 265: 2114151.

Pacheco, M.M., Mourao, M., Mantovani, E.B., Nishimoto, I.N. and Brentani, M.M. (1998) Expression of gelatinases $A$ and $B$, stromelysin- 3 and matrilysin genes in breast carcinomas: clinico-pathological correlations. Clin Exp Metastasis, 16: 577-85.

Palecek, S.P., Loftus, J.C., Ginsberg, M.H., Lauffenburger, D.A. and Horwitz, A.F. (1997) Integrin-ligand binding properties govern cell migration speed through cellsubstratum adhesiveness. Nature, 385: 537-40.

Palecek, S.P., Schmidt, C.E., Lauffenburger, D.A. and Horwitz, A.F. (1996) Integrin dynamics on the tail region of migrating fibroblasts. J Cell Sci, 109: 941-52.

Paquette, B., Bisson, M., Therriault, H., Lemay, R., Pare, M., Banville, P. and Cantin, A.M. (2003) Activation of matrix metalloproteinase-2 and -9 by 2- and 4hydroxyestradiol. J Steroid Biochem Mol Biol, 87: 6573.

Park, H.I., Jin, Y., Hurst, D.R., Monroe, C.A., Lee, S., Schwartz, M.A. and Sang, Q.X. (2003) The intermediate S1' pocket of the endometase/matrilysin-2 active site revealed by enzyme inhibition kinetic studies, protein sequence analyses, and homology modeling. J Biol Chem, 278: 51646-53.

Parmley, S.F. and Smith, G.P. (1988) Antibody-selectable filamentous fd phage vectors: affinity purification of target genes. Gene, 73: 305-18.

Parsons, J.T., Martin, K.H., Slack, J.K., Taylor, J.M. and Weed, S.A. (2000) Focal adhesion kinase: a regulator of focal adhesion dynamics and cell movement. Oncogene, 19: 5606-13.

Partridge, C.A., Phillips, P.G., Niedbala, M.J. and Jeffrey, J.J. (1997) Localization and activation of type IV collagenase/gelatinase at endothelial focal contacts. Am J Physiol, 272: L813-22.

Pasqualini, R. and Ruoslahti, E. (1996) Organ targeting in vivo using phage display peptide libraries. Nature, $\mathbf{3 8 0}$ 364-6.

Pastrnak, M. and Schultz, P.G. (2001) Phage selection for site-specific incorporation of unnatural amino acids into proteins in vivo. Bioorg Med Chem, 9: 2373-9.

Patterson, B.C. and Sang, Q.A. (1997) Angiostatinconverting enzyme activities of human matrilysin (MMP-7) and gelatinase B/type IV collagenase (MMP9). J Biol Chem, 272: 28823-5.

Patterson, M.L., Atkinson, S.J., Knauper, V. and Murphy, G. (2001) Specific collagenolysis by gelatinase A, MMP-2, is determined by the hemopexin domain and not the fibronectin-like domain. FEBS Lett, 503: 158-62.

Paulus, H. (2000) Protein splicing and related forms of protein autoprocessing. Annu Rev Biochem, 69: 44796.

Pavlaki, M. and Zucker, S. (2003) Matrix metalloproteinase inhibitors (MMPIs): the beginning of phase I or the termination of phase III clinical trials. Cancer Metastasis Rev, 22: 177-203.

Pedchenko, V., Zent, R. and Hudson, B.G. (2003) av $\{\beta\} 3$ and $\operatorname{av}\{\beta\} 5$ integrins bind both the proximal RGD site and non-RGD motifs withinnoncollagenous (NC1) domain of the a3 chain of type IV collagen: Implication for themechanism of endothelial cell adhesion. J Biol Chem, 10: 10.

Pei, D., Kang, T. and Qi, H. (2000) Cysteine array matrix metalloproteinase (CA-MMP)/MMP-23 is a type II transmembrane matrix metalloproteinase regulated by a single cleavage for both secretion and activation. $J$ Biol Chem, 275: 33988-97.

Pei, D. and Weiss, S.J. (1996) Transmembrane-deletion mutants of the membrane-type matrix metalloproteinase-1 process progelatinase $A$ and express intrinsic matrix-degrading activity. $\mathrm{J}$ Biol Chem, 271: $9135-40$.

Peng, K.W., Morling, F.J., Cosset, F.L., Murphy, G. and Russell, S.J. (1997) A gene delivery system activatable 
by disease-associated matrix metalloproteinases. Hum Gene Ther, 8: 729-38.

Peppin, G.J. and Weiss, S.J. (1986) Activation of the endogenous metalloproteinase, gelatinase, by triggered human neutrophils. Proc Natl Acad Sci U S A, 83: 4322-6.

Petitclerc, E., Boutaud, A., Prestayko, A., Xu, J., Sado, Y., Ninomiya, Y., Sarras, M.P., Jr., Hudson, B.G. and Brooks, P.C. (2000) New functions for non-collagenous domains of human collagen type IV. Novel integrin ligands inhibiting angiogenesis and tumor growth in vivo. J Biol Chem, 275: 8051-61.

Pfeifer, A., Kessler, T., Silletti, S., Cheresh, D.A. and Verma, I.M. (2000) Suppression of angiogenesis by lentiviral delivery of PEX, a noncatalytic fragment of matrix metalloproteinase 2. Proc Natl Acad Sci U S A, 97 12227-32.

Pipkorn, R., Boenke, C., Gehrke, M. and Hoffmann, R. (2002) High-throughput peptide synthesis and peptide purification strategy at the low micromol-scale using the 96-well format. J Pept Res, 59: 105-14.

Pirilä, E., Maisi, P., Salo, T., Koivunen, E. and Sorsa, T. (2001) In vivo localization of gelatinases (MMP-2 and 9) by in situ zymography with a selective gelatinase inhibitor. Biochem Biophys Res Commun, 287: 766-74.

Podgorski, I. and Sloane, B.F. (2003) Cathepsin B and its role(s) in cancer progression. Biochem Soc Symp: 26376.

Polette, M., Gilbert, N., Stas, I., Nawrocki, B., Noel, A., Remacle, A., Stetler-Stevenson, W.G., Birembaut, P and Foidart, M. (1994) Gelatinase A expression and localization in human breast cancers. An in situ hybridization study and immunohistochemical detection using confocal microscopy. Virchows Arch, 424: 641-5.

Pöllänen, J., Hedman, K., Nielsen, L.S., Dano, K. and Vaheri, A. (1988) Ultrastructural localization of plasma membrane-associated urokinase-type plasminogen activator at focal contacts. J Cell Biol, 106: 87-95.

Pöllänen, J., Saksela, O., Salonen, E.M., Andreasen, P. Nielsen, L., Dano, K. and Vaheri, A. (1987) Distinct localizations of urokinase-type plasminogen activator and its type 1 inhibitor under cultured human fibroblasts and sarcoma cells. J Cell Biol, 104: 1085-96.

Pozzi, A., LeVine, W.F. and Gardner, H.A. (2002) Low plasma levels of matrix metalloproteinase 9 permit increased tumor angiogenesis. Oncogene, 21: 272-81.

Pozzi, A., Moberg, P.E., Miles, L.A., Wagner, S., Soloway, P. and Gardner, H.A. (2000) Elevated matrix metalloprotease and angiostatin levels in integrin a 1 knockout mice cause reduced tumor vascularization. Proc Natl Acad Sci U S A, 97: 2202-7.

Prager, G.W., Breuss, J.M., Steurer, S., Mihaly, J. and Binder, B.R. (2003) Vascular endothelial growth factor (VEGF) induces rapid pro-urokinase (pro-uPA) activation on the surface of endothelial cells. Blood, 2: 2.

Primakoff, P. and Myles, D.G. (2000) The ADAM gene family: surface proteins with adhesion and protease activity. Trends Genet, 16: 83-7.

Puyraimond, A., Fridman, R., Lemesle, M., Arbeille, B. and Menashi, S. (2001) MMP-2 colocalizes with caveolae on the surface of endothelial cells. Exp Cell Res, 262: 28-36.

Puyraimond, A., Weitzman, J.B., Babiole, E. and Menashi, S. (1999) Examining the relationship between the gelatinolytic balance and the invasive capacity of endothelial cells. J Cell Sci, 112: 1283-90.

Qi, J.H., Ebrahem, Q., Moore, N., Murphy, G., ClaessonWelsh, L., Bond, M., Baker, A. and Anand-Apte, B. (2003) A novel function for tissue inhibitor of metalloproteinases-3 (TIMP3): inhibition of angiogenesis by blockage of VEGF binding to VEGF receptor-2. Nat Med, 9: 407-15.

Qian, X., Wang, T.N., Rothman, V.L., Nicosia, R.F. and Tuszynski, G.P. (1997) Thrombospondin-1 modulates angiogenesis in vitro by up-regulation of matrix metalloproteinase-9 in endothelial cells. Exp Cell Res, 235: 403-12.

Rajotte, D., Arap, W., Hagedorn, M., Koivunen, E., Pasqualini, R. and Ruoslahti, E. (1998) Molecular heterogeneity of the vascular endothelium revealed by in vivo phage display. J Clin Invest, 102: 430-7.

Ramos-DeSimone, N., Hahn-Dantona, E., Sipley, J., Nagase, H., French, D.L. and Quigley, J.P. (1999) Activation of matrix metalloproteinase-9 (MMP-9) via a converging plasmin/stromelysin-1 cascade enhances tumor cell invasion. J Biol Chem, 274: 13066-76.

Ray, J.M. and Stetler-Stevenson, W.G. (1995) Gelatinase A activity directly modulates melanoma cell adhesion and spreading. EMBO J, 14: 908-17.

Ray, S., Lukyanov, P. and Ochieng, J. (2003) Members of the cystatin superfamily interact with MMP-9 and protect it from autolytic degradation without affecting its gelatinolytic activities. Biochim Biophys Acta, 1652: 91102.

Ree, A.H., Florenes, V.A., Berg, J.P., Maelandsmo, G.M., Nesland, J.M. and Fodstad, O. (1997) High levels of messenger RNAs for tissue inhibitors of metalloproteinases (TIMP-1 and TIMP-2) in primary breast carcinomas are associated with development of distant metastases. Clin Cancer Res, 3: 1623-8.

Regen, C.M. and Horwitz, A.F. (1992) Dynamics of $\beta 1$ integrin-mediated adhesive contacts in motile fibroblasts. J Cell Biol, 119: 1347-59.

Rennert, O.M. and Anker, H.S. (1963) On the Incorporation of $5^{\prime}, 5^{\prime}, 5^{\prime}-$ Trifluoroleucine Into Proteins of E. Coli. Biochemistry, 13: 471-6.

Reynolds, L.E., Wyder, L., Lively, J.C., Taverna, D., Robinson, S.D., Huang, X., Sheppard, D., Hynes, R.O. and Hodivala-Dilke, K.M. (2002) Enhanced pathological angiogenesis in mice lacking $\beta 3$ integrin or $\beta 3$ and $\beta 5$ integrins. Nat Med, 8: 27-34.

Ridley, A.J. and Hall, A. (1992) The small GTP-binding protein rho regulates the assembly of focal adhesions and actin stress fibers in response to growth factors. Cell, 70: 389-99.

Riikonen, T., Westermarck, J., Koivisto, L., Broberg, A., Kahari, V. M. and Heino, J. (1995) Integrin $\alpha 2 \beta 1$ is a positive regulator of collagenase (MMP-1) and collagen a 1(I) gene expression. J Biol Chem, 270: 13548-52.

Ritter, L.M., Garfield, S.H. and Thorgeirsson, U.P. (1999) Tissue inhibitor of metalloproteinases-1 (TIMP-1) binds to the cell surface and translocates to the nucleus of human MCF-7 breast carcinoma cells. Biochem Biophys Res Commun, 257: 494-9.

Rodriguez-Manzaneque, J.C., Lane, T.F., Ortega, M.A., Hynes, R.O., Lawler, J. and Iruela-Arispe, M.L. (2001) Thrombospondin-1 suppresses spontaneous tumor growth and inhibits activation of matrix metalloproteinase- 9 and mobilization of vascular endothelial growth factor. Proc Natl Acad Sci U S A, 98: 12485-90.

Roeb, E., Schleinkofer, K., Kernebeck, T., Potsch, S., Jansen, B., Behrmann, I., Matern, S. and Grotzinger, J. (2002) The matrix metalloproteinase 9 (mmp-9) hemopexin domain is a novel gelatin binding domain and acts as an antagonist. J Biol Chem, 277: 50326-32. Epub 2002 Oct 15.

Rolli, M., Fransvea, E., Pilch, J., Saven, A. and FeldingHabermann, B. (2003) Activated integrin avß3 cooperates with metalloproteinase MMP-9 in regulating migration of metastatic breast cancer cells. Proc Natl Acad Sci U S A, 100: 9482-7

Rosemurgy, A., Harris, J., Langleben, A., Casper, E., Goode, S. and Rasmussen, H. (1999) Marimastat in patients with advanced pancreatic cancer: a dosefinding study. Am J Clin Oncol, 22: 247-52.

Rosenblum, G., Meroueh, S.O., Kleifeld, O., Brown, S., Singson, S.P., Fridman, R., Mobashery, S. and Sagi, I. (2003) Structural basis for potent slow binding inhibition of human matrix metalloproteinase-2 (MMP-2). J Biol Chem, 278: 27009-15. 
Rouet-Benzineb, P., Buhler, J.M., Dreyfus, P., Delcourt, A., Dorent, R., Perennec, J., Crozatier, B., Harf, A. and Lafuma, C. (1999) Altered balance between matrix gelatinases (MMP-2 and MMP-9) and their tissue inhibitors in human dilated cardiomyopathy: potential role of MMP-9 in myosin-heavy chain degradation. Eur $J$ Heart Fail, 1: 337-52.

Rowsell, S., Hawtin, P., Minshull, C.A., Jepson, H., Brockbank, S.M., Barratt, D.G., Slater, A.M., McPheat, W.L., Waterson, D., Henney, A.M. and Pauptit, R.A. (2002) Crystal structure of human MMP9 in complex with a reverse hydroxamate inhibitor. J Mol Biol, 319: 173-81.

Rudd, P.M., Mattu, T.S., Masure, S., Bratt, T., Van den Steen, P.E., Wormald, M.R., Kuster, B., Harvey, D.J., Borregaard, N., Van Damme, J., Dwek, R.A. and Opdenakker, G. (1999) Glycosylation of natural human neutrophil gelatinase $B$ and neutrophil gelatinase $B$ associated lipocalin. Biochemistry, 38: 13937-50.

Ruiz, S., Henschen-Edman, A.H., Nagase, H. and Tenner, A.J. (1999) Digestion of C1q collagen-like domain with MMPs-1,-2,-3, and -9 further defines the sequence involved in the stimulation of neutrophil superoxide production. J Leukoc Biol, 66: 416-22.

Ruoslahti, E. (1996) RGD and other recognition sequences for integrins. Annu Rev Cell Dev Biol, 12: 697-715.

Ruoslahti, E. (2000) Targeting tumor vasculature with homing peptides from phage display. Semin Cancer Biol, 10: 435-42.

Russel, M. and Model, P. (1985) Thioredoxin is required for filamentous phage assembly. Proc Natl Acad SciU S A, 82: 29-33

Saad, S., Gottlieb, D.J., Bradstock, K.F., Overall, C.M. and Bendall, L.J. (2002) Cancer cell-associated fibronectin induces release of matrix metalloproteinase-2 from normal fibroblasts. Cancer Res, 62: 283-9.

Sage, E.H., Reed, M., Funk, S.E., Truong, T., Steadele, M., Puolakkainen, P., Maurice, D.H. and Bassuk, J.A. (2003) Cleavage of the matricellular protein SPARC by matrix metalloproteinase 3 produces polypeptides that influence angiogenesis. J Biol Chem, 278: 37849-57.

Sakamoto, K., Hayashi, A., Sakamoto, A., Kiga, D., Nakayama, H., Soma, A., Kobayashi, T., Kitabatake, M., Takio, K., Saito, K., Shirouzu, M., Hirao, I. and Yokoyama, S. (2002) Site-specific incorporation of an unnatural amino acid into proteins in mammalian cells. Nucleic Acids Res, 30: 4692-9.

Salo, T., Mäkelä, M., Kylmäniemi, M., Autio-Harmainen, H. and Larjava, H. (1994) Expression of matrix metalloproteinase-2 and -9 during early human wound healing. Lab Invest, 70: 176-82.

Salonurmi, T., Parikka, M., Kontusaari, S., Pirilä, E., Munaut, C., Salo, T. and Tryggvason, K. (2004) Overexpression of TIMP-1 under the MMP-9 promoter interferes with wound healing in transgenic mice. Cell Tissue Res, 315: 27-37.

Sanceau, J., Truchet, S. and Bauvois, B. (2003) Matrix metalloproteinase- 9 silencing by RNA interference triggers the migratory-adhesive switch in Ewing's sarcoma cells. J Biol Chem, 278: 36537-46.

Sandman, K.E. and Noren, C.J. (2000) The efficiency of Escherichia coli selenocysteine insertion is influenced by the immediate downstream nucleotide. Nucleic Acids Res, 28: 755-61.

Sang, Q.A., Bodden, M.K. and Windsor, L.J. (1996) Activation of human progelatinase $\mathrm{A}$ by collagenase and matrilysin: activation of procollagenase by matrilysin. J Protein Chem, 15: 243-53.

Sasaki, T., Gohring, W., Mann, K., Maurer, P., Hohenester, E., Knauper, V., Murphy, G. and Timpl, R. (1997) Limited cleavage of extracellular matrix protein BM-40 by matrix metalloproteinases increases its affinity for collagens. J Biol Chem, 272: 9237-43.

Sato, H., Kinoshita, T., Takino, T., Nakayama, K. and Seiki, M. (1996) Activation of a recombinant membrane type 1-matrix metalloproteinase (MT1-MMP) by furin and its interaction with tissue inhibitor of metalloproteinases (TIMP)-2. FEBS Lett, 393: 101-4.

Sato, H. and Seiki, M. (1993) Regulatory mechanism of 92 $\mathrm{kDa}$ type IV collagenase gene expression which is associated with invasiveness of tumor cells. Oncogene 8: 395-405.

Schenk, S. and Quaranta, V. (2003) Tales from the crypt[ic] sites of the extracellular matrix. Trends Cell Biol, 13: 366-75.

Schmitz, R., Baumann, G. and Gram, H. (1996) Catalytic specificity of phosphotyrosine kinases Blk, Lyn, C-Src and Syk as assessed by phage display. J Mol Biol, 260 : 664-77.

Schnaper, H.W., Grant, D.S., Stetler-Stevenson, W.G., Fridman, R., D'Orazi, G., Murphy, A.N., Bird, R.E., Hoythya, M., Fuerst, T.R., French, D.L. and et al. (1993) Type IV collagenase(s) and TIMPs modulate endothelial cell morphogenesis in vitro. J Cell Physiol, 156: 235-46.

Schonbeck, U., Mach, F. and Libby, P. (1998) Generation of biologically active IL-1 $\beta$ by matrix metalloproteinases: a novel caspase-1-independent pathway of IL-1 $\beta$ processing. J Immunol, 161: 3340-6.

Scorilas, A., Karameris, A., Arnogiannaki, N., Ardavanis, A., Bassilopoulos, P., Trangas, T. and Talieri, M. (2001) Overexpression of matrix-metalloproteinase-9 in human breast cancer: a potential favourable indicator in nodenegative patients. Br J Cancer, 84: 1488-96.

Scott, C.P., Abel-Santos, E., Wall, M., Wahnon, D.C. and Benkovic, S.J. (1999) Production of cyclic peptides and proteins in vivo. Proc Natl Acad Sci U S A, 96: 1363843.

Scott, J.K. and Smith, G.P. (1990) Searching for peptide ligands with an epitope library. Science, 249: 386-90.

Seftor, R.E., Seftor, E.A., De Larco, J.E., Kleiner, D.E., Leferson, J., Stetler-Stevenson, W.G., McNamara, T.F., Golub, L.M. and Hendrix, M.J. (1998) Chemically modified tetracyclines inhibit human melanoma cell invasion and metastasis. Clin Exp Metastasis, 16: 21725.

Seftor, R.E., Seftor, E.A., Koshikawa, N., Meltzer, P.S Gardner, L.M., Bilban, M., Stetler-Stevenson, W.G. Quaranta, V. and Hendrix, M.J. (2001) Cooperative interactions of laminin 5 gamma2 chain, matrix metalloproteinase-2, and membrane type-1matrix/metalloproteinase are required for mimicry of embryonic vasculogenesis by aggressive melanoma. Cancer Res, 61: 6322-7.

Sehgal, G., Hua, J., Bernhard, E.J., Sehgal, I., Thompson, T.C. and Muschel, R.J. (1998) Requirement for matrix metalloproteinase-9 (gelatinase B) expression in metastasis by murine prostate carcinoma. Am J Pathol, 152: $591-6$.

Seltzer, J.L., Eisen, A.Z., Bauer, E.A., Morris, N.P., Glanville, R.W. and Burgeson, R.E. (1989) Cleavage of type VII collagen by interstitial collagenase and type IV collagenase (gelatinase) derived from human skin. $J$ Biol Chem, 264: 3822-6.

Seo, D.W., Li, H., Guedez, L., Wingfield, P.T., Diaz, T., Salloum, R., Wei, B.Y. and Stetler-Stevenson, W.G. (2003) TIMP-2 mediated inhibition of angiogenesis: an MMP-independent mechanism. Cell, 114: 171-80.

Severinov, K. and Muir, T.W. (1998) Expressed protein ligation, a novel method for studying protein-protein interactions in transcription. J Biol Chem, 273: 16205-9.

Shalinsky, D.R., Brekken, J., Zou, H., McDermott, C.D. Forsyth, P., Edwards, D., Margosiak, S., Bender, S., Truitt, G., Wood, A., Varki, N.M. and Appelt, K. (1999) Broad antitumor and antiangiogenic activities of AG3340, a potent and selective MMP inhibitor undergoing advanced oncology clinical trials. Ann N Y Acad Sci, 878: 236-70.

Shamamian, P., Pocock, B.J., Schwartz, J.D., Monea, S. Chuang, N., Whiting, D., Marcus, S.G., Galloway, A.C. and Mignatti, P. (2000) Neutrophil-derived serine proteinases enhance membrane type-1 matrix 
metalloproteinase-dependent tumor cell invasion Surgery, 127: 142-7.

Sheu, B.C., Hsu, S.M., Ho, H.N., Lien, H.C., Huang, S.C. and Lin, R.H. (2001) A novel role of metalloproteinase in cancer-mediated immunosuppression. Cancer Res, 61: 237-42.

Shi, Y.E., Torri, J., Yieh, L., Wellstein, A., Lippman, M.E. and Dickson, R.B. (1993) Identification and characterization of a novel matrix-degrading protease from hormonedependent human breast cancer cells. Cancer Res, 53: 1409-15.

Shimaoka, M., Salas, A., Yang, W., Weitz-Schmidt, G. and Springer, T.A. (2003) Small molecule integrin antagonists that bind to the $\beta 2$ subunit I-like domain and activate signals in one direction and block them in the other. Immunity, 19: 391-402.

Shimaoka, M. and Springer, T. A. (2003) Therapeutic antagonists and conformational regulation of integrin function. Nat Rev Drug Discov, 2: 703-16.

Shofuda, T., Shofuda, K., Ferri, N., Kenagy, R.D., Raines, E.W. and Clowes, A.W. (2004) Cleavage of focal adhesion kinase in vascular smooth muscle cells overexpressing membrane-type matrix metalloproteinases. Arterioscler Thromb Vasc Biol, 24: 839-44.

Silletti, S., Kessler, T., Goldberg, J., Boger, D.L. and Cheresh, D.A. (2001) Disruption of matrix metalloproteinase 2 binding to integrin $\alpha v \beta 3$ by an organic molecule inhibits angiogenesis and tumor growth in vivo. Proc Natl Acad Sci U S A, 98: 119-24.

Sires, U.I., Griffin, G.L., Broekelmann, T.J., Mecham, R.P., Murphy, G., Chung, A.E., Welgus, H.G. and Senior, R.M. (1993) Degradation of entactin by matrix metalloproteinases. Susceptibility to matrilysin and identification of cleavage sites. J Biol Chem, 268: 206974.

Siri, A., Knauper, V., Veirana, N., Caocci, F., Murphy, G. and Zardi, L. (1995) Different susceptibility of small and large human tenascin- $C$ isoforms to degradation by matrix metalloproteinases. J Biol Chem, 270: 8650-4

Smith, G.N., Jr., Hasty, K.A., Yu, L.P., Jr., Lamberson, K.S., Mickler, E.A. and Brandt, K.D. (1991) Cleavage of type $\mathrm{XI}$ collagen fibers by gelatinase and by extracts of osteoarthritic canine cartilage. Matrix, 11: 36-42.

Smith, G.P. (1985) Filamentous fusion phage: novel expression vectors that display cloned antigens on the virion surface. Science, 228: 1315-7.

Smith, M.M., Shi, L. and Navre, M. (1995) Rapid identification of highly active and selective substrates for stromelysin and matrilysin using bacteriophage peptide display libraries. J Biol Chem, 270: 6440-9.

Smothers, J.F. and Henikoff, S. (2001) Predicting in vivo protein peptide interactions with random phage display. Comb Chem High Throughput Screen, 4: 585-91.

Solberg, H., Romer, J., Brunner, N., Holm, A., Sidenius, N., Dano, K. and Hoyer-Hansen, G. (1994) A cleaved form of the receptor for urokinase-type plasminogen activator in invasive transplanted human and murine tumors. Int J Cancer, 58: 877-81.

Soloway, P.D., Alexander, C.M., Werb, Z. and Jaenisch, R. (1996) Targeted mutagenesis of TIMP-1 reveals that lung tumor invasion is influenced by TIMP-1 genotype of the tumor but not by that of the host. Oncogene, 13: 2307-14.

Sopata, I. and Wize, J. (1979) A latent gelatin specific proteinase of human leucocytes and its activation. Biochim Biophys Acta, 571: 305-12.

Sorsa, T., Ramamurthy, N.S., Vernillo, A.T., Zhang, X., Konttinen, Y.T., Rifkin, B.R. and Golub, L.M. (1998) Functional sites of chemically modified tetracyclines: inhibition of the oxidative activation of human neutrophil and chicken osteoclast pro-matrix metalloproteinases. $J$ Rheumatol, 25: 975-82.

Sorsa, T., Salo, T., Koivunen, E., Tyynelä, J., Konttinen, Y.T., Bergmann, U., Tuuttila, A., Niemi, E., Teronen, O., Heikkilä, P., Tschesche, H., Leinonen, J., Osman,
S. and Stenman, U.H. (1997) Activation of type IV procollagenases by human tumor-associated trypsin-2. J Biol Chem, 272: 21067-74.

Sottrup-Jensen, L. and Birkedal-Hansen, H. (1989) Human

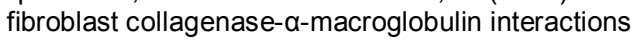
Localization of cleavage sites in the bait regions of five mammalian a-macroglobulins. J Biol Chem, 264: 393401.

Spessotto, P., Rossi, F.M., Degan, M., Di Francia, R., Perris, R., Colombatti, A. and Gattei, V. (2002) HyaluronanCD44 interaction hampers migration of osteoclast-like cells by down-regulating MMP-9. J Cell Biol, 158: 113344.

Srinivasan, G., James, C.M. and Krzycki, J.A. (2002) Pyrrolysine encoded by UAG in Archaea: charging of a UAG-decoding specialized tRNA. Science, 296: 145962.

Stacker, S.A., Achen, M.G., Jussila, L., Baldwin, M.E. and Alitalo, K. (2002) Lymphangiogenesis and cancer metastasis. Nat Rev Cancer, 2: 573-83.

Stam, J.C., Michiels, F., van der Kammen, R.A., Moolenaar, W.H. and Collard, J.G. (1998) Invasion of T-lymphoma cells: cooperation between Rho family GTPases and lysophospholipid receptor signaling. EMBO J, 17: 406674.

Stanton, H., Gavrilovic, J., Atkinson, S.J., d'Ortho, M.P., Yamada, K.M., Zardi, L. and Murphy, G. (1998) The activation of ProMMP-2 (gelatinase A) by HT1080 fibrosarcoma cells is promoted by culture on a fibronectin substrate and is concomitant with an increase in processing of MT1-MMP (MMP-14) to a 45 kDa form. J Cell Sci, 111: 2789-98.

Starckx, S., Van den Steen, P.E., Verbeek, R., van Noort, J.M. and Opdenakker, G. (2003) A novel rationale for inhibition of gelatinase B in multiple sclerosis: MMP-9 destroys a B-crystallin and generates a promiscuous $T$ cell epitope. J Neuroimmunol, 141: 47-57.

Steffensen, B., Bigg, H.F. and Overall, C.M. (1998) The involvement of the fibronectin type II-like modules of human gelatinase $A$ in cell surface localization and activation. J Biol Chem, 273: 20622-8.

Steffensen, B., Wallon, U.M. and Overall, C.M. (1995) Extracellular matrix binding properties of recombinant fibronectin type II-like modules of human 72-kDa gelatinase/type IV collagenase. High affinity binding to native type I collagen but not native type IV collagen. $J$ Biol Chem, 270: 11555-66.

Sternlicht, M.D. and Werb, Z. (2001) How matrix metalloproteinases regulate cell behavior. Annu Rev Cell Dev Biol, 17: 463-516.

Stetler-Stevenson, W.G. and Yu, A.E. (2001) Proteases in invasion: matrix metalloproteinases. Semin Cancer Biol, 11: 143-52.

Stocker, W., Grams, F., Baumann, U., Reinemer, P., GomisRuth, F.X., McKay, D.B. and Bode, W. (1995) The metzincins--topological and sequential relations between the astacins, adamalysins, serralysins, and matrixins (collagenases) define a superfamily of zincpeptidases. Protein Sci, 4: 823-40.

Stricker, T.P., Dumin, J.A., Dickeson, S.K., Chung, L., Nagase, H., Parks, W.C. and Santoro, S.A. (2001) Structural analysis of the $\alpha 2$ integrin I domain/procollagenase-1 (matrix metalloproteinase-1) interaction. J Biol Chem, 276: 29375-81.

Strongin, A.Y., Collier, I., Bannikov, G., Marmer, B.L., Grant, G.A. and Goldberg, G.I. (1995) Mechanism of cell surface activation of 72-kDa type IV collagenase. Isolation of the activated form of the membrane metalloprotease. J Biol Chem, 270: 5331-8.

Sugiura, T. and Berditchevski, F. (1999) Function of $\alpha 3 \beta 1$ tetraspanin protein complexes in tumor cell invasion. Evidence for the role of the complexes in production of matrix metalloproteinase 2 (MMP-2). J Cell Biol, 146: 1375-89.

Suyama, E., Kawasaki, H., Kasaoka, T. and Taira, K. (2003) Identification of genes responsible for cell migration by 
a library of randomized ribozymes. Cancer Res, 63 . 119-24.

Takagi, J., Petre, B.M., Walz, T. and Springer, T.A. (2002) Global conformational rearrangements in integrin extracellular domains in outside-in and inside-out signaling. Cell, 110: 599-11.

Takahashi, C., Sheng, Z., Horan, T.P., Kitayama, H., Maki, M., Hitomi, K., Kitaura, Y., Takai, S., Sasahara, R.M., Horimoto, A., Ikawa, Y., Ratzkin, B.J., Arakawa, T. and Noda, M. (1998) Regulation of matrix metalloproteinase- 9 and inhibition of tumor invasion by the membrane-anchored glycoprotein RECK. Proc Natl Acad Sci U S A, 95: 13221-6.

Takeha, S., Fujiyama, Y., Bamba, T., Sorsa, T., Nagura, H. and Ohtani, H. (1997) Stromal expression of MMP-9 and urokinase receptor is inversely associated with liver metastasis and with infiltrating growth in human colorectal cancer: a novel approach from immune/inflammatory aspect. Jpn J Cancer Res, 88: 72-81.

Takino, T., Koshikawa, N., Miyamori, H., Tanaka, M., Sasaki, T., Okada, Y., Seiki, M. and Sato, H. (2003) Cleavage of metastasis suppressor gene product KiSS1 protein/metastin by matrix metalloproteinases. Oncogene, 22: 4617-26.

Tamura, Y., Watanabe, F., Nakatani, T., Yasui, K., Fuji, M., Komurasaki, T., Tsuzuki, H., Maekawa, R., Yoshioka, T., Kawada, K., Sugita, K. and Ohtani, M. (1998) Highly selective and orally active inhibitors of type IV collagenase (MMP-9 and MMP-2): N-sulfonylamino acid derivatives. J Med Chem, 41: 640-9.

Tang, B.L. (2001) ADAMTS: a novel family of extracellular matrix proteases. Int J Biochem Cell Biol, 33: 33-44.

Tang, Y., Ghirlanda, G., Petka, W.A., Nakajima, T. DeGrado, W.F. and Tirrell, D.A. (2001) Fluorinated Coiled-Coil Proteins Prepared In Vivo Display Enhanced Thermal and Chemical Stability. Angew Chem Int Ed Engl, 40: 1494-1496.

Tang, Y. and Tirrell, D.A. (2001) Biosynthesis of a Highly Stable Coiled-Coil Protein Containing Hexafluoroleucine in an Engineered Bacterial Host. $J$ Am Chem Soc, 123: 11089-11090.

Tanzawa, K., Ishii, M., Ogita, T. and Shimada, K. (1992) Matlystatins, new inhibitors of typelV collagenases from Actinomadura atramentaria. II. Biological activities. $J$ Antibiot (Tokyo), 45: 1733-7.

Taraboletti, G., D'Ascenzo, S., Borsotti, P., Giavazzi, R., Pavan, A. and Dolo, V. (2002) Shedding of the matrix metalloproteinases MMP-2, MMP-9, and MT1-MMP as membrane vesicle-associated components by endothelial cells. Am J Pathol, 160: 673-80.

Teronen, O., Heikkilä, P., Konttinen, Y.T., Laitinen, M., Salo, T., Hanemaaijer, R., Teronen, A., Maisi, P. and Sorsa, T. (1999) MMP inhibition and downregulation by bisphosphonates. Ann N Y Acad Sci, 878: 453-65

Thrailkill, K.M., Quarles, L.D., Nagase, H., Suzuki, K., Serra, D.M. and Fowlkes, J.L. (1995) Characterization of insulin-like growth factor-binding protein 5-degrading proteases produced throughout murine osteoblast differentiation. Endocrinology, 136: 3527-33.

Tordai, H. and Patthy, L. (1999) The gelatin-binding site of the second type-II domain of gelatinase A/MMP-2. Eur J Biochem, 259: 513-8.

Toth, M., Chvyrkova, I., Bernardo, M.M., HernandezBarrantes, S. and Fridman, R. (2003) Pro-MMP-9 activation by the MT1-MMP/MMP-2 axis and MMP-3: role of TIMP-2 and plasma membranes. Biochem Biophys Res Commun, 308: 386-95.

Toth, M., Gervasi, D.C. and Fridman, R. (1997) Phorbol ester-induced cell surface association of matrix metalloproteinase- 9 in human MCF10A breast epithelial cells. Cancer Res, 57: 3159-67.

Toth, M., Sado, Y., Ninomiya, Y. and Fridman, R. (1999) Biosynthesis of $\alpha 2$ (IV) and $\alpha 1$ (IV) chains of collagen IV and interactions with matrix metalloproteinase-9. J Cell Physiol, 180: 131-9.
Tremble, P.M., Lane, T.F., Sage, E.H. and Werb, Z. (1993) SPARC, a secreted protein associated with morphogenesis and tissue remodeling, induces expression of metalloproteinases in fibroblasts through a novel extracellular matrix-dependent pathway. J Cell Biol, 121: 1433-44.

Trexler, M., Briknarova, K., Gehrmann, M., Llinas, M. and Patthy, L. (2003) Peptide ligands for the fibronectin type II modules of matrix metalloproteinase 2 (MMP-2). J Biol Chem, 278: 12241-6.

Triebel, S., Blaser, J., Reinke, H., Knauper, V. and Tschesche, H. (1992) Mercurial activation of human PMN leucocyte type IV procollagenase (gelatinase). FEBS Lett, 298: 280-4.

Turck, J., Pollock, A.S., Lee, L.K., Marti, H.P. and Lovett, D.H. (1996) Matrix metalloproteinase 2 (gelatinase A) regulates glomerular mesangial cell proliferation and differentiation. J Biol Chem, 271: 15074-83.

Turk, B.E., Huang, L.L., Piro, E.T. and Cantley, L.C. (2001) Determination of protease cleavage site motifs using mixture-based oriented peptide libraries. Nat Biotechnol, 19: 661-7.

Turunen, M.P., Puhakka, H.L., Koponen, J.K., Hiltunen, M.O., Rutanen, J., Leppänen, O., Turunen, A.M., Närvänen, A., Newby, A.C., Baker, A.H. and YläHerttuala, S. (2002) Peptide-retargeted adenovirus encoding a tissue inhibitor of metalloproteinase-1 decreases restenosis after intravascular gene transfer. Mol Ther, 6: 306-12.

Udagawa, T., Fernandez, A., Achilles, E.G., Folkman, J. and D'Amato, R.J. (2002) Persistence of microscopic human cancers in mice: alterations in the angiogenic balance accompanies loss of tumor dormancy. FASEB J, 16: 1361-70.

Uppala, A. and Koivunen, E. (2000) Targeting of phage display vectors to mammalian cells. Comb Chem High Throughput Screen, 3: 373-92.

Walchli, S., Espanel, X., Harrenga, A., Rossi, M., Cesareni, G. and van Huijsduijnen, R.H. (2004) Probing proteintyrosine phosphatase substrate specificity using a phosphotyrosine-containing phage library. J Biol Chem, 279: 311-8.

van den Oord, J.J., Paemen, L., Opdenakker, G. and de Wolf-Peeters, C. (1997) Expression of gelatinase B and the extracellular matrix metalloproteinase inducer EMMPRIN in benign and malignant pigment cell lesions of the skin. Am J Pathol, 151: 665-70.

Van den Steen, P.E., Dubois, B., Nelissen, I., Rudd, P.M., Dwek, R.A. and Opdenakker, G. (2002) Biochemistry and molecular biology of gelatinase $B$ or matrix metalloproteinase-9 (MMP-9). Crit Rev Biochem Mol Biol, 37: 375-536.

Van den Steen, P.E., Husson, S.J., Proost, P., Van Damme, J. and Opdenakker, G. (2003) Carboxyterminal cleavage of the chemokines MIG and IP-10 by gelatinase B and neutrophil collagenase. Biochem Biophys Res Commun, 310: 889-96.

Van den Steen, P.E., Proost, P., Wuyts, A., Van Damme, J. and Opdenakker, G. (2000) Neutrophil gelatinase B potentiates interleukin- 8 tenfold by aminoterminal processing, whereas it degrades CTAP-III, PF-4, and GRO- $\alpha$ and leaves RANTES and MCP-2 intact. Blood, 96: 2673-81.

Van Den Steen, P.E., Wuyts, A., Husson, S.J., Proost, P., Van Damme, J. and Opdenakker, G. (2003) Gelatinase B/MMP-9 and neutrophil collagenase/MMP-8 process the chemokines human GCP-2/CXCL6, ENA$78 / C X C L 5$ and mouse GCP-2/LIX and modulate their physiological activities. Eur J Biochem, 270: 3739-49.

van 't Veer, L.J., Dai, H., van de Vijver, M.J., He, Y.D., Hart, A.A., Mao, M., Peterse, H.L., van der Kooy, K., Marton, M.J., Witteveen, A.T., Schreiber, G.J., Kerkhoven, R.M., Roberts, C., Linsley, P.S., Bernards, R. and Friend, S.H. (2002) Gene expression profiling predicts clinical outcome of breast cancer. Nature, 415: 530-6. 
Van Wart, H.E. and Birkedal-Hansen, H. (1990) The cysteine switch: a principle of regulation of metalloproteinase activity with potential applicability to the entire matrix metalloproteinase gene family. Proc Natl Acad Sci U S A, 87: 5578-82.

Wang, L., Brock, A., Herberich, B. and Schultz, P.G. (2001) Expanding the genetic code of Escherichia coli. Science, 292: 498-500.

Wang, W., Schulze, C.J., Suarez-Pinzon, W.L., Dyck, J.R., Sawicki, G. and Schulz, R. (2002) Intracellular action of matrix metalloproteinase-2 accounts for acute myocardial ischemia and reperfusion injury. Circulation, 106: $1543-9$.

Wang, X.Q., Sun, P. and Paller, A.S. (2003) Ganglioside GM3 inhibits matrix metalloproteinase- 9 activation and disrupts its association with integrin. J Biol Chem, 278:25591-9

Wang, Z., Juttermann, R. and Soloway, P.D. (2000) TIMP-2 is required for efficient activation of proMMP-2 in vivo. $J$ Biol Chem, 275: 26411-5.

Vartio, T., Hovi, T. and Vaheri, A. (1982) Human macrophages synthesize and secrete a major 95,000dalton gelatin-binding protein distinct from fibronectin. $J$ Biol Chem, 257: 8862-6.

Vartio, T. and Vaheri, A. (1981) A gelatin-binding 70,000dalton glycoprotein synthesized distinctly from fibronectin by normal and malignant adherent cells. $J$ Biol Chem, 256: 13085-90.

Watanabe, K., Takahashi, H., Habu, Y., Kamiya-Kubushiro, N., Kamiya, S., Nakamura, H., Yajima, H., Ishii, T., Katayama, T., Miyazaki, K. and Fukai, F. (2000) Interaction with heparin and matrix metalloproteinase 2 cleavage expose a cryptic anti-adhesive site of fibronectin. Biochemistry, 39: 7138-44.

Wei, Y., Eble, J.A., Wang, Z., Kreidberg, J.A. and Chapman, H.A. (2001) Urokinase receptors promote $\beta 1$ integrin function through interactions with integrin $\alpha 3 \beta 1$. Mol Biol Cell, 12: 2975-86.

Wei, Y., Lukashev, M., Simon, D.I., Bodary, S.C. Rosenberg, S., Doyle, M.V. and Chapman, H.A. (1996) Regulation of integrin function by the urokinase receptor. Science, 273: 1551-5.

Wei, Y., Yang, X., Liu, Q., Wilkins, J.A. and Chapman, H.A. (1999) A role for caveolin and the urokinase receptor in integrin-mediated adhesion and signaling. J Cell Biol, 144: 1285-94

Veikkola, T. and Alitalo, K. (1999) VEGFs, receptors and angiogenesis. Semin Cancer Biol, 9: 211-20.

Weisberg, E., Sattler, M., Ewaniuk, D.S. and Salgia, R (1997) Role of focal adhesion proteins in signal transduction and oncogenesis. Crit Rev Oncog, 8: 34358.

Weiss, L. (1990) Metastatic inefficiency. Adv Cancer Res, 54: 159-211.

Velkov, T. and Lawen, A. (2003) Non-ribosomal peptide synthetases as technological platforms for the synthesis of highly modified peptide bioeffectorsCyclosporin synthetase as a complex example. Biotechnol Annu Rev, 9: 151-97.

Werb, Z., Tremble, P. M., Behrendtsen, O., Crowley, E. and Damsky, C. H. (1989) Signal transduction through the fibronectin receptor induces collagenase and stromelysin gene expression. J Cell Biol, 109: 877-89.

Werb, Z. (1997) ECM and cell surface proteolysis: regulating cellular ecology. Cell, 91: 439-42.

Westermarck, J. and Kähäri, V.M. (1999) Regulation of matrix metalloproteinase expression in tumor invasion. FASEB J, 13: 781-92.

Whaley, S.R., English, D.S., Hu, E.L., Barbara, P.F. and Belcher, A.M. (2000) Selection of peptides with semiconductor binding specificity for directed nanocrystal assembly. Nature, 405: 665-8.

Whittaker, M., Floyd, C.D., Brown, P. and Gearing, A.J. (1999) Design and therapeutic application of matrix metalloproteinase inhibitors. Chem Rev, 99: 2735-76.
Wielockx, B., Lannoy, K., Shapiro, S.D., Itoh, T., Itohara, S., Vandekerckhove, J. and Libert, C. (2001) Inhibition of matrix metalloproteinases blocks lethal hepatitis and apoptosis induced by tumor necrosis factor and allows safe antitumor therapy. Nat Med, 7: 1202-8.

Williams, C.S., Mann, M. and DuBois, R.N. (1999) The role of cyclooxygenases in inflammation, cancer, and development. Oncogene, 18: 7908-16.

Visse, R. and Nagase, H. (2003) Matrix metalloproteinases and tissue inhibitors of metalloproteinases: structure, function, and biochemistry. Circ Res, 92: 827-39.

Wize, J., Sopata, I., Smerdel, A. and Maslinski, S. (1998) Ligation of selectin L and integrin CD11b/CD18 (Mac-1) induces release of gelatinase B (MMP-9) from human neutrophils. Inflamm Res, 47: 325-7.

Woiwode, T.F., Haggerty, J.E., Katz, R., Gallop, M.A., Barrett, R.W., Dower, W.J. and Cwirla, S.E. (2003) Synthetic compound libraries displayed on the surface of encoded bacteriophage. Chem Biol, 10: 847-58.

Wolf, K., Mazo, I., Leung, H., Engelke, K., von Andrian, U.H., Deryugina, E.I., Strongin, A.Y., Brocker, E.B. and Friedl, P. (2003) Compensation mechanism in tumor cell migration: mesenchymal-amoeboid transition after blocking of pericellular proteolysis. J Cell Biol, 160: 267-77.

von Bredow, D.C., Cress, A.E., Howard, E.W., Bowden, G.T. and Nagle, R.B. (1998) Activation of gelatinase-tissueinhibitors-of-metalloproteinase complexes by matrilysin. Biochem J, 331: 965-72.

Wood, D.W., Wu, W., Belfort, G., Derbyshire, V. and Belfort M. (1999) A genetic system yields self-cleaving inteins for bioseparations. Nat Biotechnol, 17: 889-92.

Woods, A., McCarthy, J.B., Furcht, L.T. and Couchman, J.R. (1993) A synthetic peptide from the COOH-terminal heparin-binding domain of fibronectin promotes focal adhesion formation. Mol Biol Cell, 4: 605-13.

Worthylake, R.A. and Burridge, K. (2001) Leukocyte transendothelial migration: orchestrating the underlying molecular machinery. Curr Opin Cell Biol, 13: 569-77.

Wright, R.M., Dudas, D., Gavin, B., Dottavio, D., Hexham, J.M. and Lake, P. (2001) A high-capacity alkaline phosphatase reporter system for the rapid analysis of specificity and relative affinity of peptides from phagedisplay libraries. J Immunol Methods, 253: 223-32.

Vu, T.H., Shipley, J.M., Bergers, G., Berger, J.E., Helms, J.A., Hanahan, D., Shapiro, S.D., Senior, R.M. and Werb, Z. (1998) MMP-9/gelatinase B is a key regulator of growth plate angiogenesis and apoptosis of hypertrophic chondrocytes. Cell, 93: 411-22.

Xiong, J.P., Stehle, T., Diefenbach, B., Zhang, R., Dunker, R., Scott, D.L., Joachimiak, A., Goodman, S.L. and Arnaout, M.A. (2001) Crystal structure of the extracellular segment of integrin a V 3 . Science, 294: 339-45.

Xiong, J.P., Stehle, T., Zhang, R., Joachimiak, A., Frech, M., Goodman, S.L. and Arnaout, M.A. (2002) Crystal structure of the extracellular segment of integrin $\alpha \mathrm{V} \beta 3$ in complex with an Arg-Gly-Asp ligand. Science, 296: 151-5.

Xu, J., Rodriguez, D., Petitclerc, E., Kim, J.J., Hangai, M., Moon, Y.S., Davis, G.E., Brooks, P.C. and Yuen, S.M. (2001) Proteolytic exposure of a cryptic site within collagen type IV is required for angiogenesis and tumor growth in vivo. J Cell Biol, 154: 1069-79.

Xue, W., Mizukami, I., Todd, R.F., 3rd and Petty, H.R. (1997) Urokinase-type plasminogen activator receptors associate with $\beta 1$ and $\beta 3$ integrins of fibrosarcoma cells: dependence on extracellular matrix components. Cancer Res, 57: 1682-9.

Yakubenko, V.P., Lishko, V.K., Lam, S.C. and Ugarova, T.P (2002) A molecular basis for integrin $\alpha M \beta 2$ ligand binding promiscuity. J Biol Chem, 107: 1.

Yakubenko, V.P., Lobb, R.R., Plow, E.F. and Ugarova, T.P. (2000) Differential induction of gelatinase B (MMP-9) and gelatinase $\mathrm{A}$ (MMP-2) in T lymphocytes upon 
$\alpha(4) B(1)$-mediated adhesion to VCAM-1 and the CS-1 peptide of fibronectin. Exp Cell Res, 260: 73-84.

Yamamoto, A., Yano, S., Shiraga, M., Ogawa, H., Goto, H., Miki, T., Zhang, H. and Sone, S. (2003) A thirdgeneration matrix metalloproteinase (MMP) inhibitor (ONO-4817) combined with docetaxel suppresses progression of lung micrometastasis of MMPexpressing tumor cells in nude mice. Int $J$ Cancer, 103: 822-8.

Yan, L., Moses, M.A., Huang, S. and Ingber, D.E. (2000) Adhesion-dependent control of matrix metalloproteinase-2 activation in human capillary endothelial cells. J Cell Sci, 113: 3979-87.

Yana, I. and Weiss, S.J. (2000) Regulation of membrane type-1 matrix metalloproteinase activation by proprotein convertases. Mol Biol Cell, 11: 2387-401.

Yang, Z., Strickland, D.K. and Bornstein, P. (2001) Extracellular matrix metalloproteinase 2 levels are regulated by the low density lipoprotein-related scavenger receptor and thrombospondin 2. J Biol Chem, 276: 8403-8.

Yebra, M., Parry, G.C., Stromblad, S., Mackman, N., Rosenberg, S., Mueller, B.M. and Cheresh, D.A. (1996) Requirement of receptor-bound urokinase-type plasminogen activator for integrin $\alpha \vee \beta 5$-directed cell migration. J Biol Chem, 271: 29393-9.

Yoder, N.C. and Kumar, K. (2002) Fluorinated amino acids in protein design and engineering. Chem Soc Rev, 31: 335-41.

Yoshizaki, T., Maruyama, Y., Sato, H. and Furukawa, M. (2001) Expression of tissue inhibitor of matrix metalloproteinase-2 correlates with activation of matrix metalloproteinase- 2 and predicts poor prognosis in tongue squamous cell carcinoma. Int J Cancer, 95: 4450 .

Yu, Q. and Stamenkovic, I. (1999) Localization of matrix metalloproteinase 9 to the cell surface provides a mechanism for CD44-mediated tumor invasion. Genes Dev, 13: 35-48.

Yu, Q. and Stamenkovic, I. (2000) Cell surface-localized matrix metalloproteinase- 9 proteolytically activates TGF- $\beta$ and promotes tumor invasion and angiogenesis Genes Dev, 14: 163-76.

Yu, W., Kim, J. and Ossowski, L. (1997) Reduction in surface urokinase receptor forces malignant cells into a protracted state of dormancy. J Cell Biol, 137: 767-77.
Zamir, E. and Geiger, B. (2001) Molecular complexity and dynamics of cell-matrix adhesions. J Cell Sci, 114 3583-90.

Zeng, Z.S., Huang, Y., Cohen, A.M. and Guillem, J.G. (1996) Prediction of colorectal cancer relapse and survival via tissue RNA levels of matrix metalloproteinase-9. J Clin Oncol, 14: 3133-40.

Zhang, Z., Gildersleeve, J., Yang, Y.Y., Xu, R., Loo, J.A., Uryu, S., Wong, C.H. and Schultz, P.G. (2004) A new strategy for the synthesis of glycoproteins. Science, 303: 371-3.

Zhao, W.Q., Li, H., Yamashita, K., Guo, X.K., Hoshino, T., Yoshida, S., Shinya, T. and Hayakawa, T. (1998) Cell cycle-associated accumulation of tissue inhibitor of metalloproteinases-1 (TIMP-1) in the nuclei of human gingival fibroblasts. J Cell Sci, 111: 1147-53

Zhao, Y.G., Xiao, A.Z., Newcomer, R.G., Park, H.I., Kang, T., Chung, L.W., Swanson, M.G., Zhau, H.E., Kurhanewicz, J. and Sang, Q.X. (2003) Activation of pro-gelatinase $B$ by endometase/matrilysin-2 promotes invasion of human prostate cancer cells. J Biol Chem, 278: $15056-64$.

Zhou, Z., Apte, S.S., Soininen, R., Cao, R., Baaklini, G.Y., Rauser, R.W., Wang, J., Cao, Y. and Tryggvason, K. (2000) Impaired endochondral ossification and angiogenesis in mice deficient in membrane-type matrix metalloproteinase I. Proc Natl Acad Sci U S A, 97: 4052-7.

Zijlstra, A., Mellor, R., Panzarella, G., Aimes, R.T., Hooper, J.D., Marchenko, N.D. and Quigley, J.P. (2002) A quantitative analysis of rate-limiting steps in the metastatic cascade using human-specific real-time polymerase chain reaction. Cancer Res, 62: 7083-92.

Zucker, S., Cao, J. and Chen, W.T. (2000) Critical appraisal of the use of matrix metalloproteinase inhibitors in cancer treatment. Oncogene, 19: 6642-50.

Zucker, S., Moll, U.M., Lysik, R.M., DiMassimo, E.I., StetlerStevenson, W.G., Liotta, L.A. and Schwedes, J.W. (1990) Extraction of type-IV collagenase/gelatinase from plasma membranes of human cancer cells. Int $J$ Cancer, 45: 1137-42.

Zucker, S., Pei, D., Cao, J. and Lopez-Otin, C. (2003) Membrane type-matrix metalloproteinases (MT-MMP). Curr Top Dev Biol, 54: 1-74. 